\title{
Are Alkyne Reductions Chemo-, Regio-, and Stereoselective Enough To Provide Pure (Z)-Olefins in Polyfunctionalized Bioactive Molecules?
}

\author{
Camille Oger, Laurence Balas,* Thierry Durand, and Jean-Marie Galano
}

Institut des Biomolécules Max Mousseron, UMR CNRS 5247, Universités Montpellier 1 et 2, Faculté de Pharmacie, 15 av. Charles Flahault, Bât. D, 34093 Montpellier Cedex 05, France

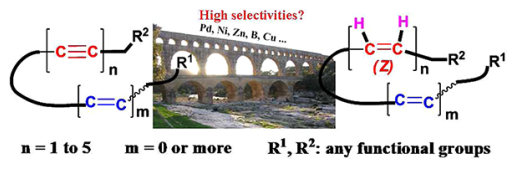

\section{CONTENTS}

1. Introduction

2. Monoalkyne Partial Reduction

2.1. Hydrogenation Using Hydrogen Gas

2.1.1. Heterogeneous Catalysts

2.1.2. Homogeneous Catalysts

2.2. Hydrogenation without Hydrogen Gas

2.2.1. Noncatalytic Systems

2.2.2. Catalytic Systems

3. Applications in Total Synthesis

3.1. The Lindlar Catalyst

3.1.1. Chemoselectivity

3.1.2. Regioselectivity-The Case of TMS-Protected Diyne

3.1.3. Application to the Preparation of Labeled Polyenes

3.2. The Rosenmund Catayst

3.2.1. Regioselectivity-The Case of TMS-Protected Diynes

3.2.2. Application to the Preparation of Polyenes

3.3. The Brown Catalyst

3.3.1. The Case of TMS-Protected Alkynes

3.3.2. Isomerization of (Z)-Double Bonds

3.3.3. Application to the Preparation of Labeled Polyenes

3.4. Activated Zinc

3.5. Hydroboration Reaction

3.5.1. Application to the Preparation of Labeled Polyenes

3.6. Titanium-Mediated Reductions

3.7. Other Methods

3.7.1. Hydroalumination

3.7.2. Tri(n-butyl)boron

3.7.3. $\mathrm{H}$-Transfer Using $(\mathrm{dba})_{3} \mathrm{Pd}_{2} \cdot \mathrm{CHCl}_{3}$ Catalyst

3.7.4. Diimide

4. Comparison of Reducing Methods

4.1. Preference for the Lindlar Catalyst

4.2. Preference for the Rosenmund Catalyst

4.3. Preference for the Brown Catalyst

4.4. Preference for a Zn-Mediated Procedure
4.5. Preference for Reduction via a Hydroboration Reaction

4.6. Preference for a Ti-Mediated Reduction

4.7. Preferences for Tri( $n$-butyl)boron 1341

4.8. Preference for Rhodium Catalyst 1341

4.9. Preference for the $\mathrm{NiCl}_{2}-\mathrm{NaBH}_{4}$ System 1341

4.10. Preference for the Hydroalumination Reaction

1341

4.11. Preference for the Trost's Catalyst 1341

4.12. Sometimes Nothing Works 1341

5. Conclusion 1341

Author Information $\quad 1342$

Corresponding Author 1342

Notes 1342

Biographies $\quad 1342$

Acknowledgments 1343

List of Abbreviations 1343

References 1343
1328

1330

1331

1331

1332

1332

1333

1333

1333

1333

1335

1336

1337

1338

1338

1338

1338

1339

1339

1339

1339

1340

1340

\section{INTRODUCTION}

Many biologically active molecules incorporate carbon-carbon double bond(s) with well-defined configurations ( $E$ or $Z$ ), such as $\beta$-carotene, ${ }^{1}$ polyene antifungal drugs, ${ }^{2,3}$ crocacine, ${ }^{4}$ but also polyunsaturated fatty acids (PUFAs), pheromones, ${ }^{5-7}$ and cruentaren. ${ }^{8}$ In the last few decades, several methods have been developed to stereochemically control the desired configurations: (i) creation of carbon-carbon double bonds (e.g., Wittig, ${ }^{9,10}$ Horner-Emmons-Wadsworth, ${ }^{11}$ Julia-Kocienski, ${ }^{12}$ Peterson, ${ }^{13}$ Takai olefination, ${ }^{14}$ olefin metathesis); ${ }^{15,16}$ (ii) cross-coupling reactions; ${ }^{17}$ (iii) reduction of alkynes; ${ }^{18-20}$ and (iv) elimination of halides. ${ }^{21}$

Most of the above-mentioned reactions led to $(E)$-isomers. Among the reactions providing $(Z)$-alkenes, we focused on the conversion of internal alkynes.

The hydrogenation or reduction of alkynes seems to be a trivial matter. Most of the student books claim that "alkynes are converted to $(Z)$-alkenes”. Thus, in most chemists' minds, there is no doubt that "the hydrogenation reaction stops cleanly when exactly one mole of hydrogen gas has been absorbed". 22

Though often perceived as simple, the semireduction reaction of alkynes becomes challenging in poly yne structures bearing functional groups and/or some other double bonds. Application to key polyfunctionalyzed precursors in a multistep 
synthesis thus required high chemo-, regio-, and stereoselective conditions. Depending on the substrate, the choice of the reduction conditions may be crucial for a successful result.

So far, there has been a great deal of interest in developing more reagents and catalysts but also new selective reducing protocols for the preparation of pure $(Z)$-alkenes. Several reviews and books have already partially covered the topic from various angles. ${ }^{20,23-27}$

The present review intends to answer some questions that organic chemists consider when writing a new retrosynthetic analysis on polyenic structures: among the plethora of methodologies developed so far, is it possible to regio-, stereo-, and chemoselectively reduce an alkyne (or more) in the presence of other double bonds? Will the functional groups be resistant to the reducing conditions?

We hope this review will serve as a handy reference for practitioners of multistep synthesis to predict which reducing reagents and conditions may give the purest alkenes (or polyenes).

The whole argument is organized in three sections. The first section includes an overview of the reducing methods described till now on monoalkynes (split up into two blocks depending whether hydrogen gas is used or not). Mechanistic information was given except when the mechanism remains unclear.

The second part is dedicated to the methods applied in the synthesis of polyenic structures with the aim to highlight chemo-, regio- and stereoselectivities, functionnalities tolerance, advantages, and/or drawbacks (side reactions...). Prevalence order was chosen to report the reducing systems. As part of our ongoing investigation on total synthesis of PUFA metabolites (endocanabinoids, ${ }^{28,29}$ neuroprostanes, ${ }^{30}$ isofuranes, ${ }^{31}$ and neuroprotectins $s^{32}$ ), the discussion particularly stressed the reduction of skipped di-, tri-, and tetraynes. However, isolated alkynes in polyenic substrates and enynes were also taken into account in order to point out the selectivities. Terminal alkynes were not considered. However, we judged it interesting to discuss the case of TMS-protected alkynes. Effectively, their reduction may provide useful vinylsilane precursors while their absence of reactivity is an elegant means to temporarily mask a terminal alkyne, with the latter being an important key intermediate in organic synthesis (for instance, organometallic cross-coupling reaction, metathesis, and click-chemistry). Besides, 1-halide-alkynes have been deliberately placed outside the scope of this review.

Some authors compared different reducing systems for the same molecule. Thus, the last section is devoted to the presentation of their preferences for the conversion of alkyne(s) function(s).

Although we have tried to make this review as comprehensive as possible, an exhaustive survey of this area is impractical. The literature has been surveyed until the beginning of 2012 .

\section{MONOALKYNE PARTIAL REDUCTION}

We wish to provide an overview of the main existing methods for the semireduction of an isolated alkyne. For each method, we place particular stress on the advantages and/or drawbacks.

Some methods were discovered more than sixty years ago and are thus well-known by organic chemists. On the other hand, some methods were reported within the last few decades only.

\subsection{Hydrogenation Using Hydrogen Gas}

2.1.1. Heterogeneous Catalysts. 2.1.1.1. Palladium Catalysts. a. Lindlar Catalyst. Catalytic hydrogenation employing Lindlar catalyst is a popular method for preparing cis-alkenes from alkynes. ${ }^{33}$ This heterogeneous catalyst consists of palladium (5\%) deposited on calcium carbonate, treated with lead acetate. ${ }^{34}$ The hydrogenation is stereoselective, providing sometimes $5-10 \%$ of $(E)$-isomer. Lead acetate poisoning led to a morphological rearrangement of the catalyst, improving the cis/trans selectivity. ${ }^{35,36}$

The overall selectivity may be increased by addition of quinoline (0.05-1 molar equiv) to inhibit alkene surface interactions. ${ }^{37}$ This can be explained by morphological changes of the palladium particles. However, in some cases the overall yield decreased due to a competition with alkyne-palladium surface interactions. ${ }^{38}$

The catalyst is commercially available, stable, and easy to handle. The reaction is usually performed under atmospheric pressure of hydrogen gas but required sometimes higher pressure $\left(5 \mathrm{~atm}^{39}\right)$. The reaction may be performed in many common organic solvents. ${ }^{20,40}$ If the reaction is not complete, the reaction can be stopped without destroying the catalyst. Then, some catalyst may be added, and the reaction is readily launched again. It is much more convenient than the use of the P2-Ni system (see sections 2.1.1.2 and 3.3).

Several functionalities, such as free ${ }^{41-45}$ and protected ${ }^{44,46,47}$ alcohols, epoxide, ${ }^{48}$ ketones, $^{49,50}$ esters, ${ }^{45,46,51}$ allylic chloride, ${ }^{52}$ cyclopropane, ${ }^{53}$ and dihydrooxazole ${ }^{54}$ functions, are tolerated. In most cases, amines were protected beforehand, ${ }^{42,43,55}$ until Campos et al. reported the use of Lindlar catalyst with 1.2 equiv of ethylenediamine for the semihydrogenation of amino-alkynes with minimal $E / Z$-isomerization and over-reduction. ${ }^{56}$ The azide group is reduced to an amine group. ${ }^{57}$

In most chemists' minds, the reduction generally halts at the alkene stage. However, if more than 1 equiv of hydrogen is absorbed, over-reduction is sometimes a serious drawback. Some isomerization may occur to some extent. ${ }^{58}$ Nevertheless, as will be discussed in section 3.1, Lindlar catalyst is widely used in total syntheses (for instance Scheme $1^{59}$ ) including poly yne structures. $^{51,59,60}$

\section{Scheme 1}
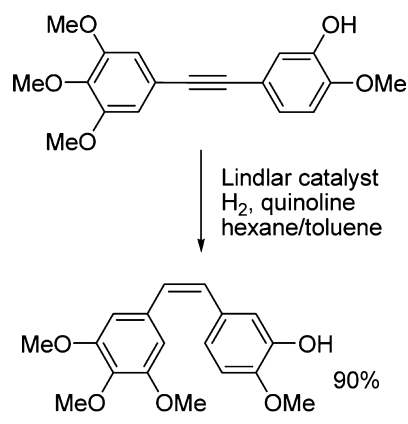

b. Modified Lindlar Catalysts. Instead of calcium carbonate in the Lindlar catalyst, the use of barium sulfate led to an efficient palladium catalyst, referred to as a "modified Lindlar catalyst" or Rosenmund catalyst.

This catalyst is usually employed in aromatic solvents (pyridine, benzene, toluene) ${ }^{47,61-63}$ or polar protic solvents $(\mathrm{MeOH}, \mathrm{EtOH}) .{ }^{64,65}$ Several groups, such as protected ${ }^{47,63,66}$ 
and free ${ }^{47,64}$ alcohols, epoxides, ${ }^{65}$ and heteroazines, ${ }^{67}$ are tolerated.

No chemoselectivity to excellent selectivity was obtained in TMS-protected alkynes. In propargylic alcohol structures, the TMS-protected alkyne function was completely hydrogenated into its corresponding TMS-alkane ${ }^{68}$ whereas only cis-hydrogenation of 2-(benzylamino)-2-(3-(trimethylsilyl)prop-2-ynyl)cyclohexanone was observed. ${ }^{69}$ It was shown that the selectivity was dramatically substrate dependent. ${ }^{70,71}$ For example, Compain et al. reported a 4/1 Z/E ratio for 2-hydroxy-2-(3trimethylsilyl)propynyl cyclohexanone hydrogenation (Scheme 2). ${ }^{72}$

Scheme 2

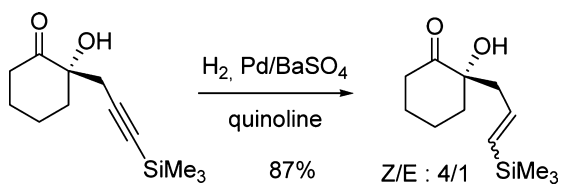

Some examples were reported with other supported palladium systems. For instance, $\mathrm{Pd} / \mathrm{CaCO}_{3}$ (without lead) in liquid polyethylene glycol, ${ }^{73} \mathrm{Pd} / \mathrm{Al}_{2} \mathrm{O}_{3},{ }^{74-76}$ or $\mathrm{Pd} / \mathrm{SiO}_{2}$ doped with $\mathrm{Hg},{ }^{77} \mathrm{Pd} / \mathrm{MCM}-41,{ }^{78,79} \mathrm{Pd}$ nanoparticles in hydrotalcite, ${ }^{80}$ and, more recently, $\mathrm{Pd} / \mathrm{Zn}$ alloy. ${ }^{81}$

c. Caubère Catalyst (Pdc). In 1984, Brunet et al. carried out the semihydrogenation of alkynes with a $\mathrm{NaH}-\mathrm{RONa}-$ $\mathrm{Pd}(\mathrm{OAc})_{2}$ mixture, entitled Pdc. ${ }^{82}$ This nonpyrophoric catalyst is employed under an atmospheric pressure of hydrogen gas at room temperature, with quinoline (quinoline/solvent ratio: 1:4 $\mathrm{v} / \mathrm{v}$ ). The authors recommend running the hydrogenation reaction in apolar solvents (hexane, octane) with terminal alkynes, and the use of ethanol, a hydrocarbon-ethanol mixture, or an ethanol-THF mixture with disubstituted alkynes.

Most examples were run with aliphatic alkynes, affording high stereoselectivities with less than $2 \%$ of over-reduced products. To date, it seems difficult to predict the application of this Pdc system to total synthesis, since only a few examples (without functionalized substrates) were investigated.

d. Palladium on Borohydride Exchange Resin (Pd-BER). The combination of borohydride exchange resin (BER, commercially available) with transition metal salts such as $\mathrm{Pd}(\mathrm{OAc})_{2}$ in $\mathrm{MeOH}$ was described in 1996 for the semihydrogenation of terminal and disubstituted alkynes in $95 \%$ ethanol. ${ }^{83}$ For unspecified reasons, CsI was poured into the hydrogenation mixture. Few nonfunctionalized alkynes, such as 2-hexyne, 3-hexyne, 1-phenyl-1-propyne, dimethyacetylene dicarboxylate, 1-hydroxy-1-phenyl-2-octyne, and 3-hydroxy-1phenyl-1-octyne, were stereoselectively hydrogenated in quantitative yield and without over-reduction. Surprisingly, this catalytic system was not further employed by chemists.

e. Palladium Nanoparticules-Tetrabutylammonium Borohydride System. In 2009, Hori et al. described a palladium nanoparticules-tetrabutylammonium borohydride system. ${ }^{84}$ Palladium nanoparticules are prepared by the reaction of $\mathrm{Pd}(\mathrm{OAc})_{2}$, oct-4-yne and $t$-BuOK in DMF. The hydrogenation reactions were performed under hydrogen gas pressure $(8 \mathrm{~atm})$ in THF. The addition of tetrabutylammonium borohydride, known as an effective stabilizer of nanoparticules, reduced the amount of isomerization as well as over-reduction. Stereoselectivities were in favor of the $(Z)$-alkenes $(>91 \%)$ with $1-$
$6 \%$ of $(E)$-alkene and/or $1-6 \%$ of alkane. In addition, it is interesting to note that the TMS-alkyne function is nicely reduced into its corresponding $(Z)$-alkene (ca. 6\% of $(E)$ isomer and $2 \%$ of alkane).

f. Montmorillonite Supported Palladium. The montmorrillonite supported bipyridinepalladium(II) acetate, bipyridinepalladium(II) choride $^{85}$ and diphenylphosphinepalladium(II) chloride ${ }^{86}$ (Scheme 3 ) were found to be efficient catalysts for the hydrogenation of alkynes, with excellent yields and without over-reduction. ${ }^{87}$

\section{Scheme 3}

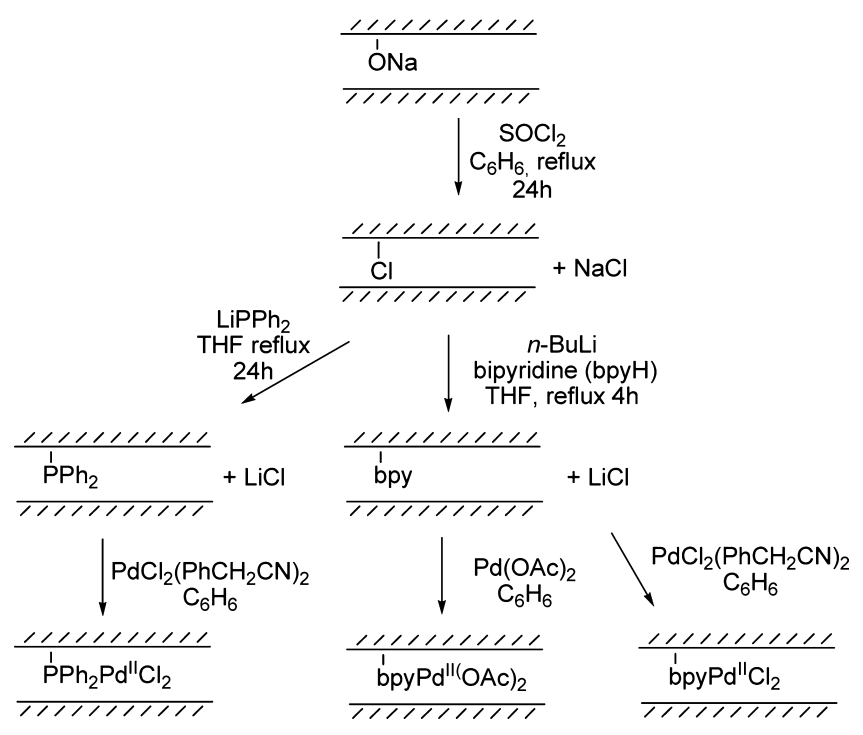

The reactions were performed in THF with hydrogen gas at rt under atmospheric pressure. However, only few examples were reported (for instance, 2-butyne-1,4-diol, diphenylacetylene, methyl phenylpropiolate, dimethyl butanedioate, ...). Therefore, prediction of the reactivity and selectivities with more functionalized substrates seems difficult.

g. Palladium on Pumice. Semihydrogenation of homopropargylic alcohols was investigated by Gruttadauria et al. as a tool for the synthesis of homoallylic alcohols, precursors for tetrahydrofuran syntheses. ${ }^{88}$ They developed a palladium on pumice catalyst, ${ }^{89}$ using 3 wt \% loading of palladium, in absolute ethanol under atmospheric pressure of hydrogen. All examples were compared to Lindlar or $\mathrm{Pd} / \mathrm{C}$ catalysts. Palladium on pumice catalyst showed better selectivities (6094\%) and a much faster reactivity. However, over-reduced products were detected.

Using a metal loading of $0.5 \%$ or $1.5 \%,{ }^{90}$ and a base (pyridine, triethylamine, ethylenediamine, 2.5-20\% with respect of the substrate), a good stereoselectivity was obtained (in absolute ethanol or THF), albeit with $4-37 \%$ of overreduction. This notable drawback may explain why nobody used this catalytic system in organic synthesis. The authors stated that the activity and selectivity of palladium on pumice may be due to the presence of sodium ions in the pumice structure. $^{90}$ In the meantime, Guczi et al. proved that containing copper systems are more active and selective toward phenylacetylene. $^{91}$

h. Palladium-Polyethyleneimine Complex. $\mathrm{Pd} / \mathrm{C}-$ ethylenediamine complex was largely studied for the stability of $O$ benzyl or $\mathrm{N}-\mathrm{CBz}$ groups, ${ }^{92-94}$ reduction of aromatic carbonyl 
Scheme 4

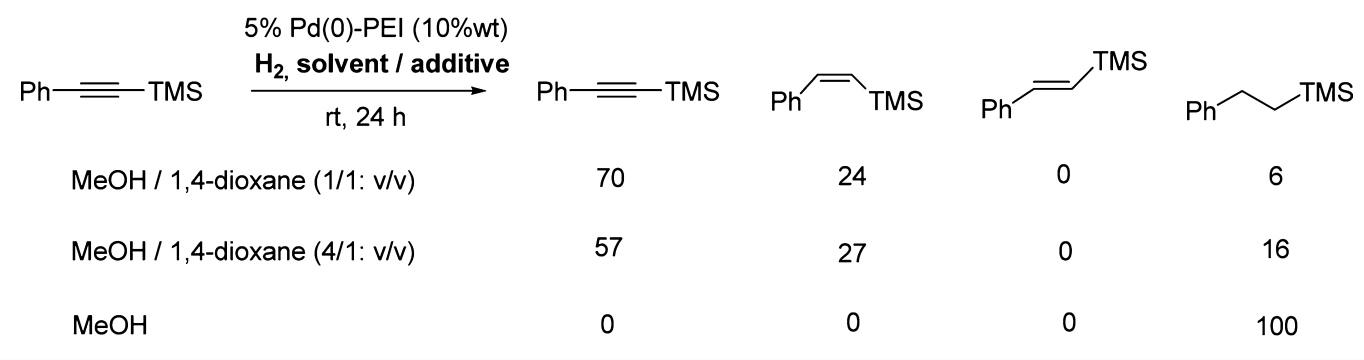

to benzyl alcohols, ${ }^{95}$ hydrogenolysis of terminal epoxides, ${ }^{96}$ reduction of nitro ${ }^{97}$ or azido groups, ${ }^{98,99}$ as well as alkene reduction ${ }^{98-100}$ and, recently, for the semihydrogenation of alkynes. ${ }^{101}$ It is noteworthy that an internal alkyne was fully reduced to an alkane. ${ }^{102}$

The above-mentioned complex was modified to a novel $\operatorname{Pd}(0)$-polyethyleneimine catalyst, prepared by the introduction of palladium acetate, polyethyleneimine (PEI) branched polymer, in $\mathrm{MeOH} / 1$,4-dioxane under hydrogen atmosphere. Good to excellent stereoselectivities were obtained ( $>94 \%$, only 6 examples with few traces of isomerization or over-reduction byproducts). No trace of over-reduction was observed with a styryl methyl ketone, albeit a poor selectivity $(36: 58 \mathrm{Z} / \mathrm{E})$. Besides, 1-phenyl-2-trimethylsilylacetylene provided a bad chemoselectivity together with over-reduction (Scheme 4). ${ }^{103}$ It must be mentioned that TBS-protected or Bn-protected alcohols tolerate these reaction conditions.

A postulated mechanistic image was described by the authors to rationalize the chemoselectivity of the reducing system. The complex formation, between $\operatorname{Pd}(0)$ metal and nitrogen atoms of PEI produced a $\mathrm{Pd}(0)-\mathrm{PEI}$ complex possessing a gentle wire-gauze-like equilibrium structure. Thanks to this PEI-gauze complex, alkenes are kept away whereas alkynes can easily access the catalytic site (Figure 1).

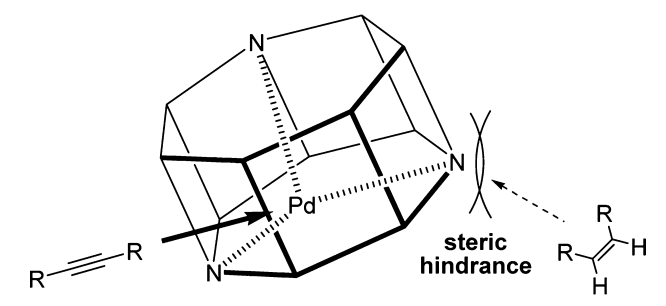

Figure 1.

i. Other Palladium Catalysts. Other palladium catalytic systems were described in the literature more recently. To date, only the semihydrogenation reaction of terminal alkynes ${ }^{104-109}$ was investigated. Thus, it may be interesting to apply such catalytic systems to internal alkyne semireduction.

2.1.1.2. Nickel Catalysts. a. P2-Ni Catalyst (Brown Catalyst). During World War II, H. C. Brown and H. I. Schlesinger noticed that sodium borohydride reacted with firstrow transition metal salts (cobalt, uranium, iron, nickel, or copper), leading to the formation of finely divided black precipitates. ${ }^{110}$ In aqueous media, a granular black material is formed from nickel acetate and sodium borohydride, named $\mathrm{P} 1-\mathrm{Ni},{ }^{111}$ as active as Raney nickel, for the reduction of double bonds. ${ }^{112}$ In the 1960s, it was found that the solvent played an important role for the formation of the catalyst, since replacement of water with ethanol led to a different reactivity for the two olefin functionalities in a same compound. ${ }^{113,114}$ This novel catalytic system was referred to as $\mathrm{P} 2-\mathrm{Ni}^{111}$

Different nickel salts were tested $\left(\mathrm{NiNO}_{3}, \mathrm{NiCl}_{2}, \mathrm{NiSO}_{4}\right.$, $\left.\mathrm{Ni}(\mathrm{OAc})_{2}\right)$. The best results were obtained with nickel acetate, which was thus chosen as the standard metal source. ${ }^{115}$ Investigation of a series of amines (quinoline, pyridine, ethylenediamine, piperidine, morpholine, triethylamine) as catalyst modifiers showed ethylenediamine (eda) to be the most effective for the production of pure $(Z)$-alkenes.

The P2-Ni catalyst is sensitive to oxygen. Thus, it has to be prepared before use and cannot be stored. One of its advantages is that the starting materials are rather inexpensive and common. Simple aliphatic alkynes gave semihydrogenation in good to excellent stereoselectivities (97:1 to 200:1 $\mathrm{Z} / \mathrm{E}$ ) with $2-4 \%$ of alkanes only. ${ }^{116}$

A typical preparation involved the addition of an ethanolic solution of $\mathrm{NaBH}_{4}\left(\mathrm{NaOH}\right.$ solution of $\mathrm{NaBH}_{4}$ could also be used $)^{117,118}$ into a suspension of $\mathrm{Ni}(\mathrm{OAc})_{2} \cdot 4 \mathrm{H}_{2} \mathrm{O}$ in ethanol under hydrogen atmosphere. A black suspension evolved, and then ethylenediamine was added followed by the addition of the alkyne.

Nowadays, with the Lindlar catalyst, the Brown catalyst is the most often used reducing system for the semihydrogenation of alkynes, tolerating alkenes, ${ }^{119}$ amides, ${ }^{120}$ acetal, ${ }^{121,122}$ oxazole, ${ }^{120}$ benzyl group, ${ }^{120}$ silylated ether, ${ }^{123,124}$ and free hydroxyl groups. ${ }^{123,124}$ Further comments on P2-Ni catalyzed hydrogenations are given in section 3.3.

b. Dispersed Nickel on Graphite. The in situ reduction of $\mathrm{NiBr}_{2} \cdot 2 \mathrm{DME}$ by potassium graphite afforded a highly nonpyrophoric dispersed nickel on graphite surface (Ni-Gr1). In the presence of ethylenediamine, Ni-Grl was used as a catalyst for the semihydrogenation of alkynes. ${ }^{125}$ However, few examples are reported with functionalized alkynes (propargylic ether and ester). Good to excellent selectivities (>94:6 Z/E ratio) and good yields (>86\%) were observed.

Treatment of Ni-Grl with water removed excess of the pyrophoric potassium graphite and provided a second generation catalyst (Ni-Gr2), which achieved good stereoselectivities in most cases. ${ }^{126}$ However, the major drawback of this reducing catalyst is the amount of over-reduction (10-50\% of alkane) and the high pressure which is required (30 atm).

c. Caubère Catalyst (Nic). The hydrogenation reactions with the $\mathrm{NaH}-\mathrm{RONa}-\mathrm{Ni}(\mathrm{OAc})_{2}$ system $^{127}$ (Nic) were performed in $\mathrm{EtOH}$ and allowed good to excellent regioselectivities in moderate to good yields. Nic is as efficient as $\mathrm{P} 2-\mathrm{Ni}$ catalyst. However, only butyn-1,4-diol was studied as a functionalized alkyne. This probably explains why this methodology has not been applied in total synthesis till now.

d. Nickel on Borohydride Exchange Resin ( $\left.\mathrm{Ni}_{2} \mathrm{~B}-\mathrm{BER}\right) . \mathrm{Ni}_{2} \mathrm{~B}-$ $B E R$ was prepared from $\mathrm{Ni}(\mathrm{OAc})_{2}$ and borohydride exchange resin (BER) in $\mathrm{MeOH} .{ }^{128}$ Reduction of nitro compounds, ${ }^{128}$ alkyl halides, ${ }^{129}$ and azides ${ }^{130}$ was realized using this $\mathrm{Ni}_{2} \mathrm{~B}-\mathrm{BER}$ 
system, avoiding thereby the presence of such functional groups for the semihydrogenation of alkynes. Seven examples of internal alkynes were reported by Choi et al. ${ }^{131}$ For example, propargylic alcohols were reduced with excellent stereoselectivity and without traces of alkanes. A protected TMSalkyne function was selectively semihydrogenated into its corresponding TMS-alkene although with a moderate yield only (43\%, Scheme 5$).^{71}$ In this latter paper, $\mathrm{Ni}_{2} \mathrm{~B}-\mathrm{BER}$ was more efficient than the $\mathrm{Pd}-\mathrm{BaSO}_{4} /$ quinoline and $\mathrm{Pd}-\mathrm{CO}_{3} /$ $\mathrm{PbO}$ catalyst. To date, no comparison with $\mathrm{P} 2-\mathrm{Ni}$ was given.

\section{Scheme 5}

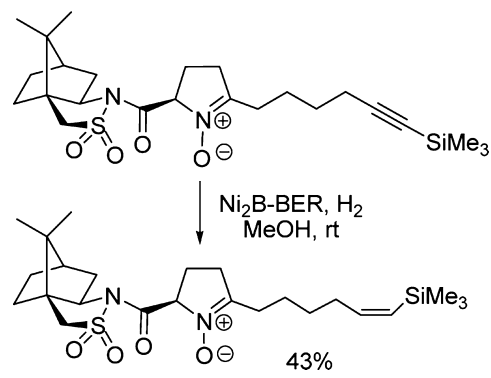

2.1.2. Homogeneous Catalysts. Besides heterogeneous transition metal catalysis, many homogeneous transition metal complexes were reported. To date, reaction mechanisms remain obscure.

This section focuses on homogeneous catalytic systems using molecular hydrogen for the semihydrogenation of alkynes. ${ }^{24}$

2.1.2.1. Using Palladium. Zero-valent palladium catalysts bearing bidentate nitrogen ligand were reported to form $\mathrm{C}-\mathrm{C}$ bonds $^{132}$ or to hydrogenate alkynes ${ }^{133-135}$ under mild conditions ( 1 atm of $\mathrm{H}_{2}, 20{ }^{\circ} \mathrm{C}$, in THF) with high stereoselectivity. Aliphatic alkynes and a homopropargylic alcohol were reduced with an excellent $(Z)$-selectivity and without over-reduction. Aromatic alkynes showed good selectivities, albeit with $3-13 \%$ of alkane. ${ }^{24,132,135}$

In the mechanism proposed by van Laren et al. ${ }^{135}$ in 1999 (Scheme 6), complex I, containing a rigid bidendate nitrogen ligand, was considered as a precatalyst. This precatalyst was isolated with an electron-deficient alkene as ancillary ligand. Then, the proposed catalytic cycle expected that the electrondeficient alkene can be substituted by the alkyne. After hydrogenation of the alkyne, a subsequent substitution on complex III is performed by an excess of alkyne.

In 2005, after kinetics and spectroscopic studies on 4-octyne hydrogenation, using $\left[\mathrm{Pd}\left(\left(\left(\mathrm{CF}_{3}\right)_{2} \mathrm{C}_{6} \mathrm{H}_{3}\right)-\mathrm{Bian}\right)(\mathrm{MA})\right]$ catalyst $($ Bian $=$ bis (aryl)acenaphtalenequinonediimine), Kluwer et al. proposed another catalytic cycle. ${ }^{134}$ Their study showed that the hydrogenation proceeds by pairwise addition of the two hydrogen atoms to the substrate, which may involve a palladium(alkyne)-hydride species B (Scheme 7). It should be mentioned that $(E)$-alkene signals were not detected, suggesting that the percentage of $(E)$-alkene obtained experimentally could not come from direct hydrogenation.

Pelizzi and co-workers described a palladium(II) complex using different hydrazinic ligands, ${ }^{136-138}$ and they studied their activity mainly on alkenes and terminal alkynes. Only two examples of disubstituted alkynes were reported by the authors. While reduction of 4-octyne showed a nice stereoselectivity, the reduction of diphenylacetylene afforded $40 \%$ of the alkane byproduct. $^{139}$

\section{Scheme 6}

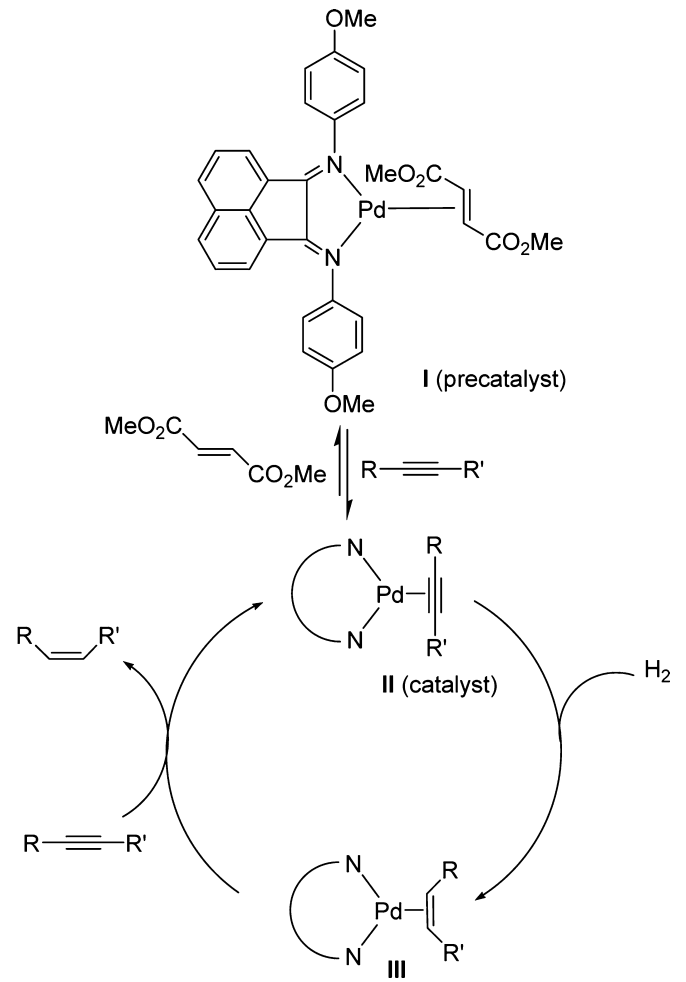

Scheme 7

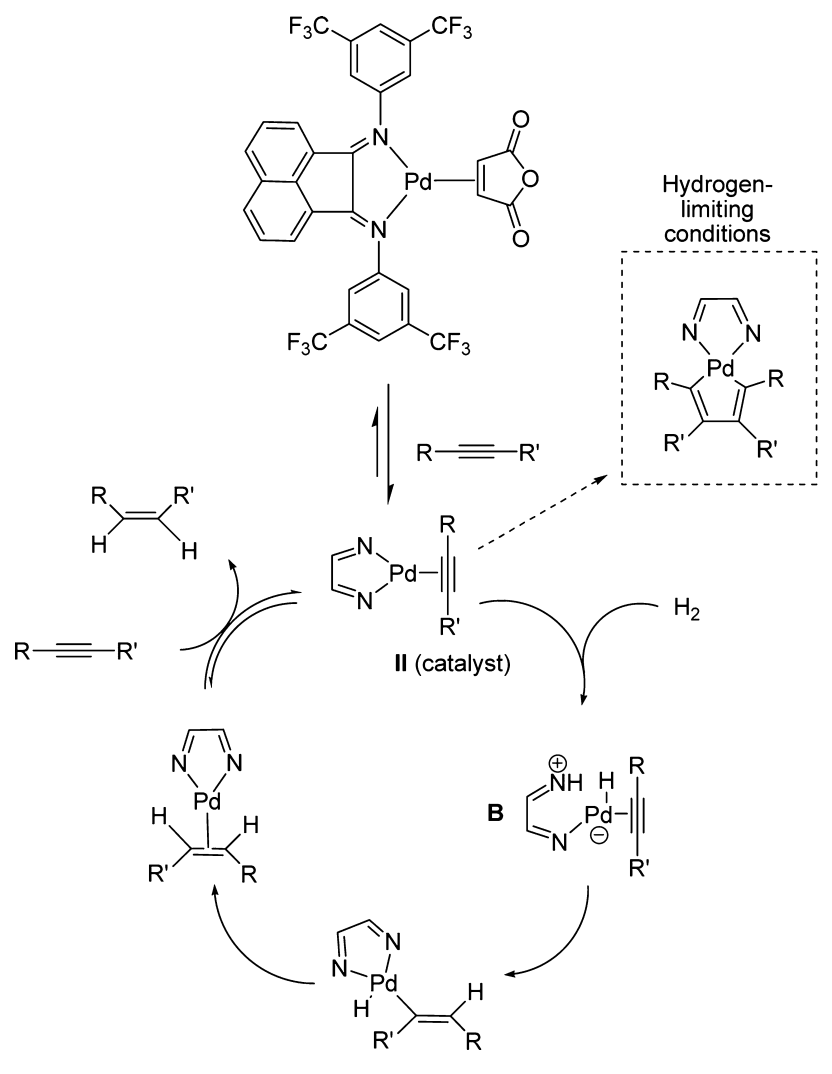

Sagar et al. described palladium(II) complexes of s-triazines and anthranilic acid for the reduction of aromatic nitro compounds, benzaldehyde, alkenes, and alkynes. ${ }^{140}$ Concerning alkyne reduction, diphenylacetylene was reduced using $\left[\mathrm{Pd}_{2}(\mathrm{OEt}-\mathrm{T})_{2} \mathrm{Cl}_{2}\right]$ catalyst $(T=2$-o-hydroxyphenyl-4-amino- 
6-alkoxy-1,3,5-triazine), in DMF, at $25{ }^{\circ} \mathrm{C}$ and using $1 \mathrm{~atm}$ of hydrogen gas. However, poor selectivity was obtained. Indeed, after $40 \mathrm{~min}$, diphenylacetylene was fully reduced into a mixture of cis-stilbene (around 85\%) and 1,2-diphenylethane (around $15 \%)$.

2.1.2.2. Using Ruthenium. $\left[\mathrm{RuH}\left(\mathrm{PMe}_{2} \mathrm{Ph}\right)_{5}\right] \mathrm{PF}_{6}$, an homogeneous cationic ruthenium(II) complex, selectively reduced alkynes without hydrogenating the intermediate alkenes. ${ }^{141}$ Unfortunately, only one example of disubstituted alkyne (2butyne) was studied. An excellent stereoselectivity was obtained in methanol without over-reduction, even after complete consumption of the alkyne. ${ }^{141}$ This $\left[\mathrm{RuH}\left(\mathrm{PMe}_{2} \mathrm{Ph}\right)_{5}\right] \mathrm{PF}_{6}$ catalyst is not commercially available yet. Its preparation was reported by Ashworth et al. ${ }^{142}$

A phosphine-deficient analog of the $\left[\mathrm{RuH}\left(\mathrm{PMe}_{2} \mathrm{Ph}\right)_{5}\right] \mathrm{PF}_{6}$ catalyst such as $\left[\mathrm{RuH}(\mathrm{COD})\left(\mathrm{PMe}_{2} \mathrm{Ph}\right)_{3}\right] \mathrm{PF}_{6}(\mathrm{COD}=1,5-$ cyclooctadiene) was also studied by the authors. ${ }^{143}$ The $\left[\mathrm{RuH}(\mathrm{COD})\left(\mathrm{PMe}_{2} \mathrm{Ph}\right)_{3}\right] \mathrm{PF}_{6}$ catalyst is not commercially available yet. It was prepared according to the procedure described by Ashworth et al. ${ }^{144}$

This catalyst hydrogenated both alkenes and alkynes under mild conditions $\left(1 \mathrm{~atm}, 25{ }^{\circ} \mathrm{C}\right.$ ). However, the addition of 2 equiv of $\mathrm{PMe}_{2} \mathrm{Ph}$ inhibits alkene hydrogenations. Thus, reduction of alkynes chemoselectively halts at the stage of the pure $(Z)$-alkenes, without contamination by over-reduction byproducts.

Lough et al. suggested that $\left[\mathrm{RuH}\left(\mathrm{PMe}_{2} \mathrm{Ph}\right)_{3}\right]^{+}$species was active for the hydrogenation of both alkenes and alkynes, whereas a $\left[\mathrm{RuH}\left(\mathrm{PMe}_{2} \mathrm{Ph}\right)_{4}\right]^{+}$species only hydrogenates alkynes. $\left[\mathrm{RuH}\left(\mathrm{PMe}_{2} \mathrm{Ph}\right)_{5}\right] \mathrm{PF}_{6}$ needs to lose $\mathrm{PMe}_{2} \mathrm{Ph}$ to generate the active species. ${ }^{145}$

The mechanism described in Scheme 8 proposed for a terminal alkyne might be useful to understand the catalytic chemistry of ruthenium. ${ }^{145}$

\section{Scheme 8}

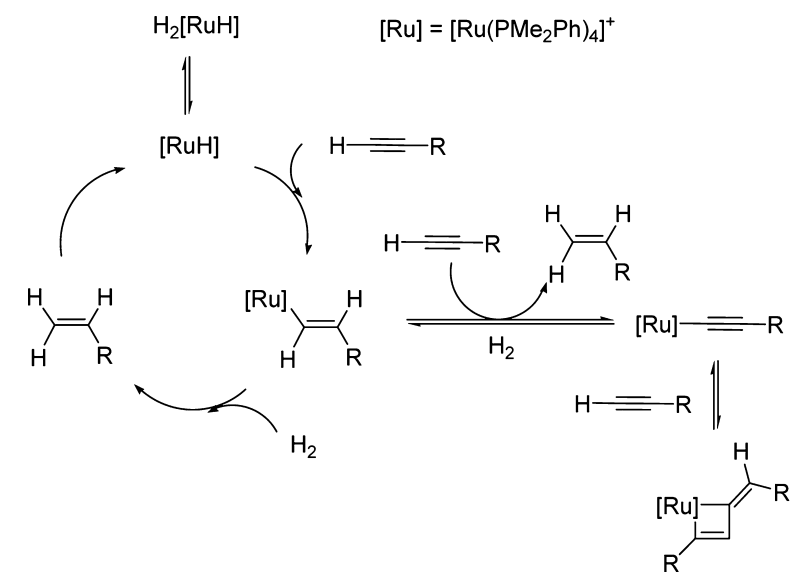

2.1.2.3. Using chromium. Chromium tricarbonyl catalysts with the generic formula $\left[\mathrm{Cr}(\mathrm{CO})_{3}(\right.$ arene $\left.)\right]$ were developed for alkynes semihydrogenation by Sodeoka et al. ${ }^{146}$ The use of methylbenzoate or naphthalene as arene source allowed the cishydrogenation of some aliphatic alkynes, aromatic alkynes, and propargylic alcohols with excellent stereoselectivity. Neither over-reduction nor isomerization were observed, even at high hydrogen pressure $\left(20-70 \mathrm{~kg} / \mathrm{cm}^{2}\right)$.

However, it is noteworthy that conjuguated dienes and $\alpha, \beta$ unsaturated compounds were readily hydrogenated using the same chromium catalysts. ${ }^{147,148}$ As an example, chromium catalyzed hydrogenation was performed in the synthesis of the principal pheromone of the Douglas fir tussock moth (Scheme 9). The $(Z)$-olefin was obtained stereospecifically (100\% stereoselectivity) in $88 \%$ yield. ${ }^{146}$

Scheme 9

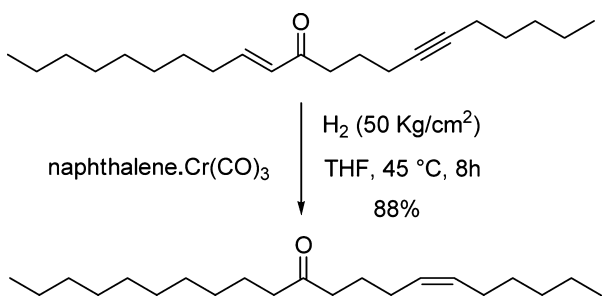

In addition, although commercially available, a serious drawback of hydrogenations using these chromium tricarbonyl catalysts stems from the need for high pressures $(20-70 \mathrm{~kg} /$ $\mathrm{cm}^{2}$ ) at high temperatures $\left(45-120{ }^{\circ} \mathrm{C}\right)$.

2.1.2.4. Using Rhodium. The Schrock-Osborn cationic rhodium catalyst ${ }^{24}\left[\mathrm{Rh}(\mathrm{ndb}) \mathrm{L}_{n}\right]^{+} \mathrm{X}^{-}$, wherein $\mathrm{ndb}$ is norbornadiene, $\mathrm{L}$ is a phosphine ligand, and $\mathrm{X}^{-}$is a weakly coordinating anion, is the most reported rhodium catalyst for the semihydrogenation of internal alkynes, with good stereoselectivities (around 95\%). ${ }^{149}$

The $[\mathrm{Rh}(\mathrm{ndb})(\mathrm{dppb})] \mathrm{BF}_{4}$ was successfully employed in the synthesis of sarcodictyins (Scheme 10). ${ }^{150}$ Isolated alkene, TIPS, and free hydroxyl groups were resistant. ${ }^{151}$

\section{Scheme 10}
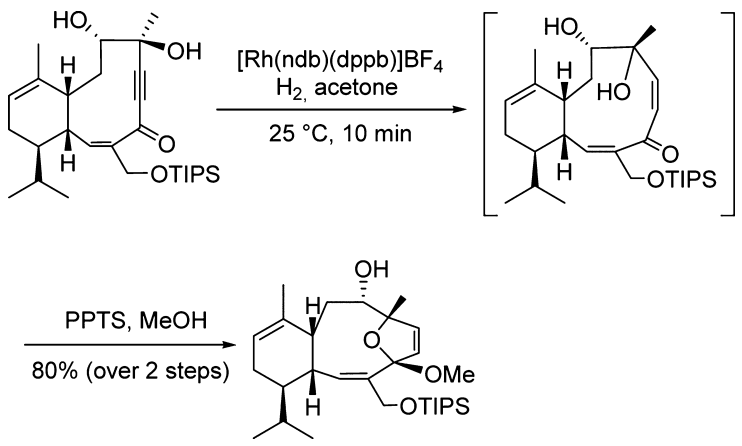

The mechanism for olefin hydrogenations proposed by Schrock et al. may be extended to alkyne hydrogenation. ${ }^{149}$

More recently, Sagar et al. ${ }^{152}$ described rhodium(I) complexes for the reduction of aromatic nitro compounds, benzaldehyde, alkenes, and alkynes. Concerning alkyne reduction, diphenylacetylene was reduced using $\left[\mathrm{Rh}(\mathrm{Az}) \mathrm{CO}_{2}\right]$ catalyst $(\mathrm{Az}=$ azobenzene $)$, in $\mathrm{DMF}$, at $25{ }^{\circ} \mathrm{C}$ and using $1 \mathrm{~atm}$ of hydrogen gas. However, poor selectivity was obtained. Indeed, diphenylacetylene was first reduced to cis-stilbene and then to 1,2-diphenylethane, leading to a complex mixture of diphenylacetylene/cis-stilbene/1,2-diphenylethane.

Other rhodium complexes were described and essentially tested on alkenes ${ }^{153}$ and terminal alkynes. ${ }^{154-156}$ Until now, the only example ${ }^{155}$ of internal alkyne yielded the corresponding fully hydrogenated substrate.

2.1.2.5. Using Niobium. Very recently, the use of a niobium(III) imido-complex for the (Z)-selective hydrogenation of 1-phenylprop-1-yne was described. ${ }^{157}$ The hydro- 
genation was performed using 1 equiv of molecular hydrogen, an excess of $\mathrm{CO}$ (12 equiv), and $20 \mathrm{~mol} \%$ of (BDI) Nb( $\mathrm{N} t$ $\mathrm{Bu})(\mathrm{CO})_{2}$ catalyst $\left(\mathrm{BDI}=N, N^{\prime}\right.$-bis $(2,6$-diisopropylphenyl $)-\beta$ diketimidate), in benzene to afford (Z)- $\beta$-methylstyrene in $75 \%$ yield. The mechanism proposed by the authors, supported by DFT calculations, involves the coordination of an alkyne to form a metalacyclopropene $\mathrm{Nb}(\mathrm{V})$ complex, followed by $\sigma$ bond metathesis with hydrogen gas and subsequent reductive elimination. Excess $\mathrm{CO}$ is required for the catalyst stability.

2.1.2.6. Using Vanadium. Toste recently reported a cationic vanadium bisimido-complex $\left[\mathrm{V}(\mathrm{N} t-\mathrm{Bu})_{2}(\mathrm{PMe})_{3}\right]\left[\mathrm{Al}(\mathrm{PFTB})_{4}\right]$ $(\mathrm{PFTB}=$ perfluoro-tert-butoxide $) .{ }^{158}$ Moderate to excellent yields were obtained, excepted for TMS-alkynes (44\%). Two reasonable mechanisms were suggested, and further studies are ongoing. ${ }^{158}$

\subsection{Hydrogenation without Hydrogen Gas}

2.2.1. Noncatalytic Systems. 2.2.1.1. Via Hydrometalation. a. Using Boron. The hydroboration-protonolysis sequence affords $(Z)$-alkenes with good to excellent stereoselectivities and yields depending on the hydroborating reagent and reaction conditions.

Since no hydrogen gas is needed, the reaction conditions present the advantage to be less dangerous than the heterogeneous and homogeneous metal-catalyzed hydrogenation. However, extreme care must be taken to exclude moisture. $^{159}$

Since the impressive masterpiece of Brown et al. ${ }^{160-168}$ on the hydroboration reaction, considerable efforts are focused on the development of novel hydroborating agents or catalysts with the aim of improving the stereoselectivity, chemoselectivity, and handling (less moisture sensitive, etc). ${ }^{169}$

A second hydroboration can be prevented if a sterically hindered borane is used. Suitable for this purpose are diisopentylborane, dicyclohexylborane, and thexylhaloboranemethyl sulfide, ${ }^{170,171}$ for example.

Over the years, many metal-catalyzed hydroboration reactions were reported: ${ }^{172,173}$ rhodium, ${ }^{173}$ nickel, ${ }^{172,174}$ iridium, ${ }^{175}$ copper, ${ }^{176,177}$ zirconium, ${ }^{178,179}$ gold, ${ }^{180}$ and titanium. ${ }^{181}$ Some mechanistic considerations were reviewed for olefins, ${ }^{173}$ and a few studies were reported starting with alkynes. For instance, Scheme 11 illustrates a hydroboration mechanism using pinacolborane and catalyzed by a zirconium complex (Schwartz reagent). ${ }^{173}$

Protonolysis (or deuteriolysis) also appeared to be a critical step. Thus, protonolysis of alkenyldialkylborane used to be performed with a 7- to 9 -fold excess of acetic acid. ${ }^{162}$ Later on,

\section{Scheme 11}

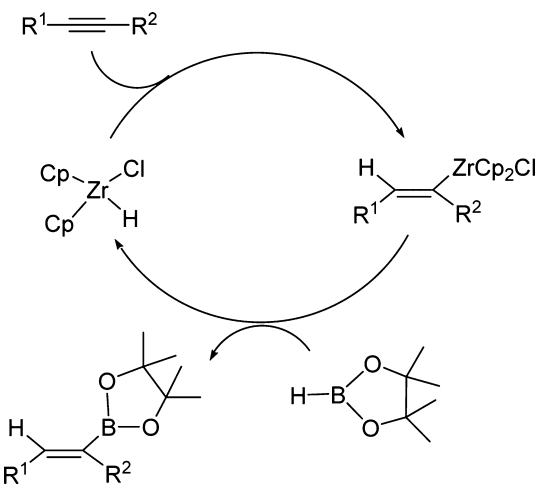

Brown et al. ${ }^{182}$ developed an improved procedure for acidsensitive derivatives using 1 equiv of methanol at $65{ }^{\circ} \mathrm{C}$. In the case of steric hindrance, addition of $1 \mathrm{~mol} \%$ pivalic acid in methanol nicely speeds up the protonolysis. ${ }^{162}$ It is noteworthy that alkenylboranes are resistant to basic conditions.

Since many functionalities are tolerated, the hydroborationprotonolysis process was widely used in total synthesis of natural products (see section 3.5). ${ }^{183-185}$ However, some chemoselectivities may differ when choosing the catalyzed or uncatalyzed hydroboration reaction conditions. For instance, ${ }^{186}$ a terminal double bond readily reacted in a rhodium-catalyzed hydroboration at $0{ }^{\circ} \mathrm{C}$ while it survived at $\mathrm{rt}$ without the rhodium catalyst (Scheme 12). ${ }^{186}$ By contrast, a ketone

\section{Scheme 12}

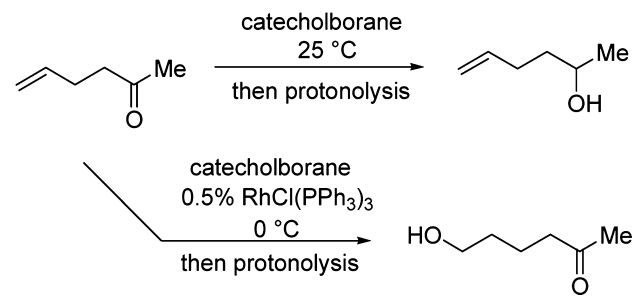

function was resistant using rhodium catalyst while it was reduced to alcohol without metal catalyst (Scheme 12). The resistance of double bonds seems substrate and reagent dependent. $^{173}$

In addition, the hydroboration reaction of terminal alkynes followed by the reaction of a Grignard reagent furnished $(Z)$ alkenes, allowing introduction of alkyl groups (moderate yields). ${ }^{187}$ Hydroboration of TMS-alkynes afforded ( $Z$ )-vinylsilanes in isomerically pure form. ${ }^{70,188}$

As will be discussed in section 3, the hydroborationprotonolysis sequence is widely used in total synthesis. An example of partial monoalkyne reduction is outlined in Scheme $13 .^{184}$

\section{Scheme 13}
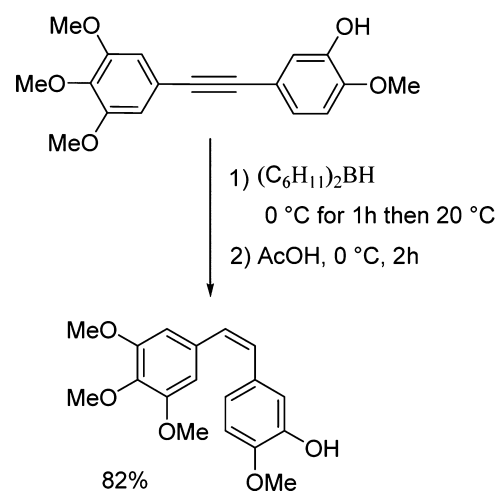

b. Using Copper. In the late 1970s, a few copper-containing hydride species such as $\left(\mathrm{KCuH}_{2}\right)_{n}{ }^{189}$ from in situ $\mathrm{KBu}_{2} \mathrm{BH}+$ $\mathrm{CuI}$ or $\mathrm{CuH}-\mathrm{HMgI}^{190}$ from in situ $\mathrm{MgH}_{2}+\mathrm{CuI}$ were reported as reducing agents. Whereas the $\left(\mathrm{KCuH}_{2}\right)_{n}$ system showed poor stereoselectivities, the $\mathrm{MgH}_{2}-\mathrm{CuI}$ system seems rather interesting (good yields, no over-reduction, excellent stereoselectivities since no $(E)$-isomer is detected). However, except alkyl groups, there was no sensitive group on the molecules. 
Thus, a large excess of magnesium dihydride may cause undesired side reactions with functionalized alkynes.

Besides, alkynes with electron-withdrawing substituents such as acetylenic sulfones were converted into their corresponding cis-vinylic sulfones at $\mathrm{rt}$ using the hydrosilane $\mathrm{Et}_{2} \mathrm{MeSiH}$ with the divalent copper salt $\mathrm{Cu}\left(\mathrm{BF}_{4}\right)_{2}$. The postulated active species is an in situ generated copper(II) hydride complex. ${ }^{191}$

The commercially available triphenylphosphinecopper hydride hexamer $\left[\left(\mathrm{Ph}_{3} \mathrm{P}\right) \mathrm{CuH}\right]_{6}$, also known as Stryker's reagent, is a source of hydride which chemoselectively hydrometalates both terminal and internal alkynes. ${ }^{192}$ The reaction proceeds in benzene with moderate to high yields. However, for some substrates, byproducts are observed resulting from overreduction (up to $16 \%$ ) or from the fragmentation of the starting material $(40-50 \%)$ in the case of unprotected propargyl alcohols. ${ }^{192}$

Many functions are well-tolerated. For instance OEE, OCOPh, OBn, and OAc ${ }^{192}$ but also organic halides, ${ }^{193}$ sulfonate groups, ${ }^{193}$ OTBS, ${ }^{194,195}$ COOEt, ${ }^{194,195}$ and nonconjugated ketones. ${ }^{194}$ Isolated double bonds in the presence of excess phosphine are not hydrogenated, even under forcing conditions. ${ }^{196-198}$ By contrast, activated alkenes such as enones are readily reduced, affording either the product of conjugated reduction or complete 1,4- and 1,2-reduction to the saturated ketone ${ }^{199}$ or alcohol, depending on the reaction conditions. ${ }^{193,196,197,200}$ Propargylic ketones suffer from a double Stryker-type reaction, ${ }^{201}$ leading to the saturated ketone in good yield (65\%, Scheme 14$)$.

\section{Scheme 14}

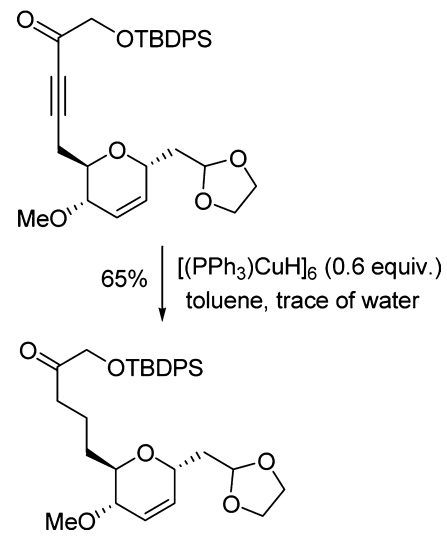

Terminal propargyl acetate undergoes a $\mathrm{S}_{\mathrm{N}} 2^{\prime}$-reduction to the corresponding allene rather than hydrocupration. ${ }^{192,202}$ Similarly, in situ generated ${ }^{203} \mathrm{Cu}-\mathrm{H}$ species converted propargyl oxiranes ${ }^{204}$ or carbonates into allenes.

The corresponding deuterated complex $\left[\left(\mathrm{Ph}_{3} \mathrm{P}\right) \mathrm{CuD}\right]_{6}$ was also readily synthesized. ${ }^{205}$ However, no example of reduction of alkyne functions was found in the literature.

In addition, NHC-carbene ligands have shown promising activities. ${ }^{204,206,207}$ Thanks to NHC-stabililized copper hydride species, Mankad et al. ${ }^{207}$ showed evidence of the hydrocupration step by isolating a vinylcuprate for the first time (Scheme 15).

c. Using Aluminum. Many aluminum hydrides were used as hydrometalating agents ${ }^{208}$ of terminal or internal alkynes (DIBAL-H, LiAlH $\left.4, \mathrm{HAl}\left[\mathrm{N}\left(\mathrm{CHMe}_{2}\right)_{2}\right]_{2}, \ldots\right)$. Subsequently, hydrolysis or deuterolysis of the intermediate alkenylalanates
Scheme 15

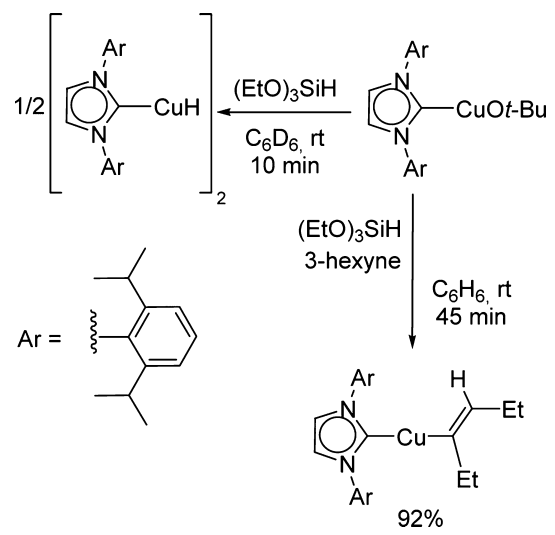

provides an efficient access to alkenes. However, alkenylalanates can dimerized to afford 1,3-butadienes. ${ }^{209}$

Interestingly, hydroalumination promoted by catalytic amounts of $\mathrm{Cp}_{2} \mathrm{TiCl}_{2}$ furnished ${ }^{210,211}$ the $(Z)$-isomer (THF, reflux) while in the absence of $\mathrm{Cp}_{2} \mathrm{TiCl}_{2}$ the hydroalumination of a $\gamma$-alkynol in diglyme at $150{ }^{\circ} \mathrm{C}$ (as preconized ${ }^{212}$ by Rossi et al.) led stereoselectively to the opposite $(E)$-alkene (Scheme 16). ${ }^{210,212}$

\section{Scheme 16}

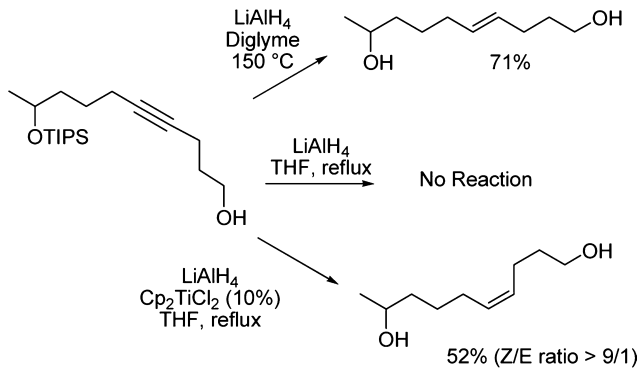

The titanium-mediated reaction with alkyl disubstituted acetylenes proceeds in high yield and high stereoselectivity $(>95 \%)$ at $\mathrm{rt}$ using a 1:1 molar ratio of alumino hydride complexes to alkyne. ${ }^{208,213,214}$ It is supposed to proceed via a hydrotitanation, followed by transmetalation to alkenylalane. ${ }^{208,210}$ It is noteworthy that the yields decrease with excess $\mathrm{Cp}_{2} \mathrm{TiCl}_{2}{ }^{213}$

Unfortunately, non-negligible amounts of the over-reduced product (up to $15 \%$ ) are sometimes observed. ${ }^{208,213}$ The same problem was encountered with the use of an equimolar ratio of $\mathrm{TiCl}_{4}-\mathrm{LiAlH}_{4}$ (1 equiv), even at low temperature $\left(-40^{\circ} \mathrm{C}\right) .^{215}$

A mechanism similar to the $\mathrm{Cp}_{2} \mathrm{TiCl}_{2}$-catalyzed hydromagnesiation $^{216}$ and hydrozincation ${ }^{217}$ was postulated by Campagne and co-workers (Scheme 17). ${ }^{210}$ This mechanism is adapted from the one described by Ashby et al. ${ }^{208}$ for hydroalumination of alkenes in the presence of $\mathrm{Cp}_{2} \mathrm{TiCl}_{2}$. $\mathrm{Cp}_{2} \mathrm{TiCl}_{2}$ is reduced in situ with $\mathrm{LiAlH}_{4}$ to titanium-hydride. This hydride undergoes hydrotitanation of the alkyne. The cisalkenyltitanium species then undergoes a transmetalation with $\mathrm{LiAlH}_{4}$ to generate the cis-alkenylaluminate product and regenerate titanium-hydride.

Application to semireduction of nonprotected $\beta$ - or $\gamma$ alkynols (4 equiv of $\mathrm{LiAlH}_{4}$, THF, reflux) showed moderated stereoselectivity (ca. 9/1) and moderate to good yields (54$82 \%){ }^{210}$ By contrast, the reduction of $\alpha$-alkynol was not 
Scheme 17

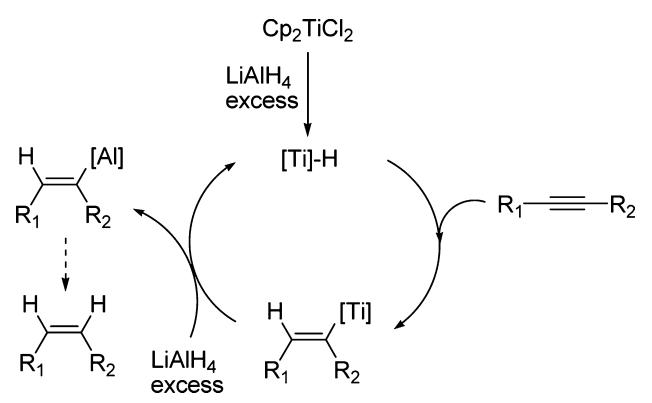

stereoselective $(Z / E 1 / 1,54 \%$ yield), certainly resulting from a competition between the catalyzed and noncatalyzed processes. $^{210}$ In addition, TMS-alkynes react slowly even at $60^{\circ} \mathrm{C}$, providing the expected $(Z)$-alkenes in $35 \%$ yield only, contaminated by their over-reduced analogs $(15 \%) .^{208}$

Free $\mathrm{OH}$, benzyl, thioacetal, and acetal groups are resistant to these harsh conditions. ${ }^{210}$ However, the excess of $\mathrm{LiAlH}_{4}$ prevents the presence of many functionalities, ${ }^{218}$ such as esters, ketones, lactones, carboxylic acid, epoxides, and aldehydes, but also amides, nitriles, halides, and nitro compounds. Depending on the reaction conditions, some silylated alcohol protections (at least OTIPS group) may also be cleaved at reflux. ${ }^{210}$ Alkenes, dienes, and ketones are readily reduced at $\mathrm{rt}^{219}$

Similarly, replacement of $\mathrm{LiAlH}_{4}$ with $\mathrm{Et}_{3} \mathrm{Al}$ was also reported as an alternative for obtaining a regio- and stereoselective hydroalumination of disubstituted acetylenes. ${ }^{220}$

$\mathrm{LiAlH}_{4}$ (0.5-1.0 equiv) was also used in combination with a stoichiometric or catalytic amount of low valent transition metal halides $\left(\mathrm{FeCl}_{3}, \mathrm{FeCl}_{2}, \mathrm{NiCl}_{2}, \mathrm{VCl}_{3}, \mathrm{CrCl}_{3}\right.$, or $\left.\mathrm{MnCl}_{2}\right)$. However, except for the cases of $\mathrm{FeCl}_{2}$ with phenylacetylene and of $\mathrm{NiCl}_{2}$ with nonfunctionalized aliphatic alkynes, no selectivity in the product distribution was observed. ${ }^{221}$

It is noteworthy that a slight isomerization to the more stable trans-isomer did occur with longer reaction times at $\mathrm{rt}^{221}$ In addition, the stereoselectivity and the chemoselectivity (observation of over-reduction side reactions) dramatically depend on the transition metal halide, the ratio of reagent to substrate, and the reaction conditions. ${ }^{221}$ Application to functionalized molecules is severely limited, since many functional groups may react with the metal hydride.

d. Hydrozincation. Gao et al. ${ }^{217}$ achieved $\mathrm{Cp}_{2} \mathrm{TiCl}_{2}-$ catalyzed hydrozincation of internal alkynes with zinc hydride reagents generated in situ from $\mathrm{ZnX}_{2}(\mathrm{X}=\mathrm{Cl}, \mathrm{Br}, \mathrm{I})$ and $\mathrm{MH}$ $(\mathrm{M}=\mathrm{Li}, \mathrm{Na}, \mathrm{K})$. The $\mathrm{ZnI}_{2}-\mathrm{LiH}(1: 2.2)$ in THF proved to be the most effective combination for obtaining the $(E)$ alkenylzinc. Quench with water or deuterium oxide provided $(Z)$-alkenes, albeit sometimes contaminated by the $(E)$-isomer (ca. 2-3\%). The role of the titanium catalyst is essential for the progress of the reaction.

As aforementioned for aluminum, the mechanism of hydrozincation follows the same cycle (Scheme 17). It is suggested ${ }^{217}$ that $\mathrm{Cp}_{2} \mathrm{TiCl}_{2}$ is reduced with $\mathrm{ZnHX}$ to yield a titanium hydride which undergoes hydrotitanation.

A TMS-alkyne was reduced to its corresponding ( $Z$ )vinylsilane with good yield (87\%) and good $(Z)$-stereoselectivity, although $2 \%$ of the $(E)$-isomer was also observed. ${ }^{217}$

The chemoselectivity of organozinc reagents is compatible with several functional groups, such as esters and even aldehydes. Surprisingly, a few functional groups, such as aryl bromide, ester, and isolated olefin, which may be reduced with hydride species and/or a low-valent titanium, survived the reaction conditions ( $\mathrm{THF}, \mathrm{rt}, \mathrm{ZnI}_{2} / \mathrm{LiH} / \mathrm{Cp}_{2} \mathrm{TiCl}_{2}=1: 2.2: 0.1$ ). However, while terminal acetylene failed ${ }^{217}$ to undergo the hydrozincation, 1,3-dienes ${ }^{222}$ readily react, providing allylzinc reagents.

Note that caution must be paid when launching the reactions, since the $\mathrm{LiH}$ powder (used in excess herein) ignites spontaneously in moist air and reacts vigorously with water. This may explain why such a methodology has not been elected by other chemists.

e. Hydromagnesiation. The direction and selectivity depend on the nature of the catalyst, the structure of the starting alkyne, and the hydromagnesiation reagent used.

Disubstituted acetylenes react with $i-\mathrm{BuMgBr}$ in the presence of a catalytic amount of $\mathrm{Cp}_{2} \mathrm{TiCl}_{2}\left(\mathrm{Et}_{2} \mathrm{O}, \mathrm{rt}\right)$ to produce $(E)$ alkenyl Grignard reagents ${ }^{223}$ in excellent yields, and thus, after hydrolysis, $(Z)$-olefins of high purity are obtained. ${ }^{224}$ This hydromagnesiation (via transmetalation of the alkenyltitanocene, as previously described for aluminum and zinc) ${ }^{216}$ also proceeds highly selectively with TMS-alkynes, leading to vinylsilanes. Alkenes and 1,3-dienes are also reduced. ${ }^{224}$ Hydromagnesiation of alkynylsilanes gave stereoselectively $(Z)-\alpha$-silyl Grignard reagents. ${ }^{216,225}$ In addition, the presence of functionalities that can react with Grignard reagents is obviously excluded. For instance, $\mathrm{EtMgBr}$ (ca. 2 equiv) and catalytic $\mathrm{Cp}_{2} \mathrm{TiCl}_{2}$ readily reacts with aldehydes and ketones. ${ }^{226}$ The intermediate species may be trapped by $\mathrm{CO}_{2}$ or TMSCl, leading to lactones or silanes.

Besides, although an excess of $i$ - $\mathrm{BuMgCl}$ was employed, the formation of the vinylmagnesium from a conjugated $(E)$ enyne $^{227}$ showed an excellent chemoselectivity for the alkyne function, even in the presence of another isolated double bond. In addition, neither regio- nor stereoisomers were detected. Excellent chemoselectivity toward alkynes in the presence of other isolated olefins was also achieved by Kociensky. ${ }^{228}$

2.2.1.2. In Situ Generated Hydrogen Gas. Yus and coworkers described reducing agents synthesized from nickel(II) chloride dihydrate, an excess of lithium powder, and a catalytic amount of arene (naphthalene or 4,4'-di-tert-butylbiphenyl: DTTB). ${ }^{229-231}$ Aromatic hydrocarbon catalysts act as an electron carrier and undergo reduction by lithium to give monoanion radical or dianion proned for the reduction of $\mathrm{Ni}(\mathrm{II})$ to $\mathrm{Ni}(0)$. Moreover, hydrogen gas was formed in situ by reaction of lithium with the water present in the nickel salt. The use of $\mathrm{NiCl}_{2} \cdot 2 \mathrm{D}_{2} \mathrm{O}$ permitted the introduction of a deuterium atom. When $1.5-2.5$ equiv of nickel salts and $17 \mathrm{~mol} \%$ of naphthalene were used, alkenes, ${ }^{232}$ as well as alkynes, were reduced into alkanes. ${ }^{233}$ When the number of equivalents of nickel salt is decreased to 1.0 or 0.5 equiv, alkynes were partially hydrogenated, leading to $(Z)$-olefins with good yield. ${ }^{233}$ In 2005 , DTTB appears to form fine nickel(0) nanoparticules ${ }^{234}$ and was used instead of naphthalene for the semireduction of alkynes. $^{235}$ Propargylic and homopropargylic alcohols were reduced with moderate to good yields, and functionalities such as methyl ether, $O$-benzyl, or tertiary amines were tolerated. ${ }^{235}$

It must be underlined that excess lithium may be a serious drawback for sensitive substrates.

2.2.1.3. Hydrolysis of Metallacyclopropene Complexes. a. Using Zinc. Activated zinc in the presence of an alcoholic solvent reduces alkynes to cis-alkenes stereospecifically.

The zinc/copper couple is now commercially available, but freshly prepared zinc reagent is often more active. ${ }^{218}$ Activated zinc couple can also be prepared using $\mathrm{Zn}$ powder activated 
with 1,2-dibromoethane, lithium dibromocuprate, or chlorhydric acid. ${ }^{236}$ The advantage of $\mathrm{Zn}(\mathrm{Cu})$ couple over the zinc metal activated in situ by 1,2-dibromoethane is that it is a storable form of activated zinc which is easily prepared and handled. ${ }^{218}$

In addition, Boland et al. have developed a $\mathrm{Zn}(\mathrm{Cu} / \mathrm{Ag})$ system $^{237}$ which is the most used $\mathrm{Zn}(\mathrm{Cu})$ system nowadays, even if this catalyst is pyrophoric.

$(Z)$-Alkenes using a $\mathrm{Zn}(\mathrm{Cu})$ couple $^{238}$ were obtained in boiling $\mathrm{MeOH}$, and long reaction times were sometimes needed $(1-24 \mathrm{~h}) .^{239}$

Free hydroxyl groups, silylated protected alcohols, ${ }^{240-242}$ as well as one or more alkenes were tolerated. ${ }^{243-247}$ Terminal alkynes were protected from semireduction thanks to a TMS group. ${ }^{236}$ It is noteworthy that a $\mathrm{Zn}(\mathrm{Cu})$ couple was found to be a convenient and efficient reagent for reduction of epoxides to olefins and of $\alpha, \beta$-unsaturated ketones to satured ketones. ${ }^{248}$

Toward the synthesis of 4,5-dihydrooxoepins, White and coworkers found that Rieke zinc was a selective reducing agent of alkynes to cis-alkenes, which allowed the reduction of enynes, diynes, and propargylic alcohols. ${ }^{249}$ Rieke zinc is a highly reactive and finely divided zinc slurry prepared by reduction of zinc salts according to Rieke et al. ${ }^{250}$ Good stereoselectivities were observed ( $Z / E$ ratio: 19/1), with good yields and in a short reaction time (around $30 \mathrm{~min}$ ). ${ }^{249}$ The reactions were performed in a mixture THF/ $\mathrm{MeOH} / \mathrm{H}_{2} \mathrm{O}$ at $65{ }^{\circ} \mathrm{C}$. Overreduction was not detected.

These zinc-mediated reductions were widely used in total synthesis as discussed in section 3.4. ${ }^{251}$ It is noteworthy that, toward the synthesis of the phoslactomycins $\mathrm{C} 1-\mathrm{C} 11$ subunit, Cossy and co-workers used $\mathrm{Zn}$ powder activated with 1,2dibromoethane (Scheme 18). ${ }^{251}$

\section{Scheme 18}
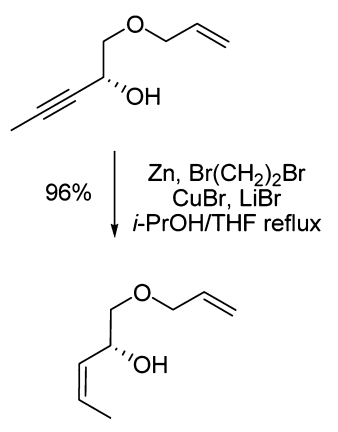

Little work has been done to determine the mechanism of this reduction reaction.

In Boland's mechanism, alkynes react with $\mathrm{Zn}(0)$ to form a zinc cyclopropene complex after two successive single electron tranfers (Scheme 19). ${ }^{243}$

b. Using Titanium. At $-78{ }^{\circ} \mathrm{C}, i-\mathrm{PrMgCl}$ (2.5 equiv) reacts with $\mathrm{Ti}(\mathrm{O} i-\mathrm{Pr})_{4}(1.0$ equiv) to form a low valent titanium alkoxide (as its alkenyl or propylene complex), which, upon addition of an alkyne ( 0.8 equiv), is converted to titanacyclopropene by a ligand exchange reaction (Scheme $20) .{ }^{252}$ Quench with water provides $(Z)$-alkenes in excellent yields and stereoselectivity $(Z / E>99 / 1)$ with no overreduction while quench with deuterium oxide allows the incorporation of two deuterium atoms. ${ }^{252}$ Hungerford et al. $^{253-256}$ applied this methodology to the formation of various
Scheme 19

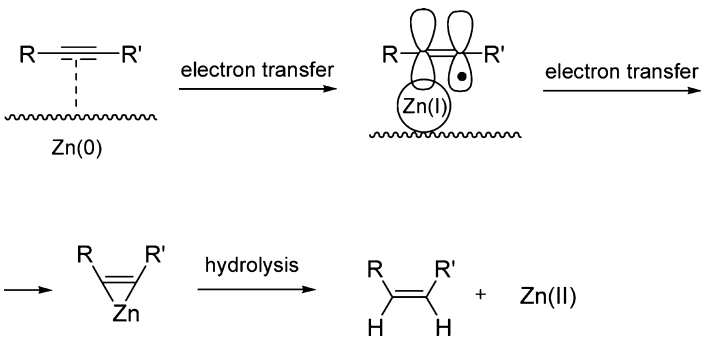

Scheme 20
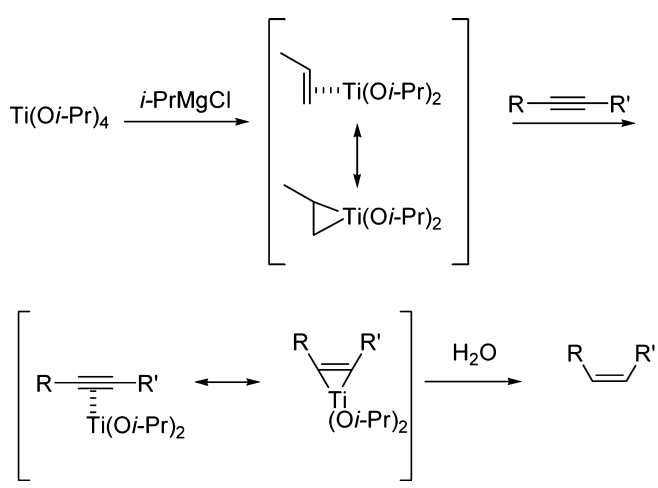

alkenes, and among them many are precursors of natural products (see section 3.6).

Excess Grignard reagent was used to ensure a complete reaction and may bother the presence of sensitive groups. Harada nicely utilized this drawback to synthesize allylic alcohols from alkynes in a one pot procedure (Scheme 21). ${ }^{252}$

Scheme 21

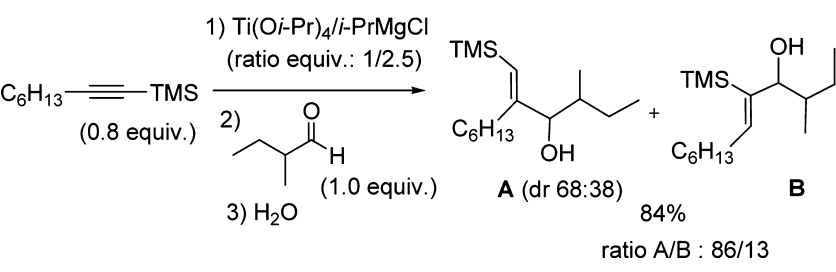

Recently, Dickson et al. described the reduction of solidsupported diaryl acetylenes ${ }^{257}$ using $\mathrm{Ti}(\mathrm{Oi}-\mathrm{Pr})_{4}(2$ equiv) and $n$-BuLi (4 equiv), in THF at $-78{ }^{\circ} \mathrm{C}$ to $\mathrm{rt}$.

2.2.1.4. Using Diimide. The reduction of multiple bonds by diimide was discovered at the beginning of the last century (1905). ${ }^{258,259}$ However, since diimide is unstable, it must be generated in situ. Many precursors and reaction conditions have been suggested. ${ }^{218,260-263}$ It has been widely used in organic synthesis.

Triple bonds are much more reactive than double bonds, but olefins and conjugated dienes readily react, leading to the saturated analogs. ${ }^{218}$ The reactivity of the double bonds depends on their strain and substitution. ${ }^{264,265}$ Thus, since many papers described an efficient reduction of alkenes, ${ }^{26,263,266-268}$ in some cases it may be difficult to predict chemoselectivity for the reduction of alkynes in the presence of alkenes, mainly if they are activated (conjugated or allylic position). In addition, polar bonds such as $\mathrm{C}=\mathrm{O}$ or $\mathrm{C}=\mathrm{N}$ are slowly reduced by diimide or do not react at all. ${ }^{258}$ Besides, in competition with the reduction of carbon-carbon $\pi$-systems, 
diimide undergoes a more facile disproportionation reaction to produce nitrogen and hydrazine. The use of considerable excess of diimide precursor is thus required and, unfortunately, favors over-reduction of alkynes (or 1-haloalkynes).

The substrate may contain many highly reactive functional groups, including $\mathrm{N}-\mathrm{O}$ or $\mathrm{O}-\mathrm{O}$ bonds, alkyl halides, phosphorus and sulfur containing systems, and carbonyl, hydroxyl, and benzyl groups. ${ }^{218}$

This methodology may also be applied to the semireduction of multiple bonds on solid-supported substrates, ${ }^{269,270}$ and for tritium labeling. ${ }^{271}$ In addition, it is noteworthy that the hydrogenation of bis(trimethylsilyl)acetylene, bis(trimethylsilyl)-1,3-diynes, and related silylalkynes using diimide prepared in situ from $p$-toluenesulfonyl hydrazide in diglyme provided the corresponding saturated compounds. ${ }^{272}$

Stereochemical studies on the reduction of alkenes have led to the suggestion that cis-diimide is a reactive hydrogen-transfer reagent. It proceeds through a concerted six-member transition state, to yield to cis-addition products (Scheme 22). ${ }^{273-275}$

Scheme 22

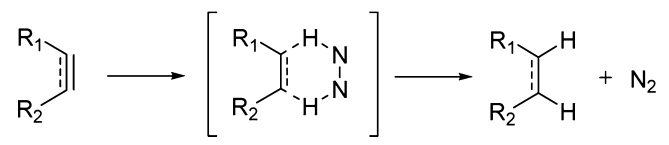

2.2.2. Catalytic Systems. Catalytic transfer hydrogenation of unsaturated compounds using organic hydrogen donors has been widely studied as an attractive method of reduction, owing to its operational simplicity and environmental friendly properties of the hydrogen donors. The reaction can be carried out under both homogeneous ${ }^{276}$ and heterogeneous ${ }^{275}$ metal catalyses, ${ }^{40}$ although both methods have been plagued by the formation of the $Z / E$ mixture and/or over-reduction.

Besides, during the selective reduction of the alkyne function, a benzyl ether group ${ }^{277}$ (even in seemingly vigorous conditions), a methyl ester, ${ }^{278-280} \mathrm{THP}^{281}$ and $\mathrm{TBS}^{278}$ ethers, nitriles, ${ }^{282}$ and nitro groups ${ }^{283}$ remained intact and free hydroxyl groups ${ }^{278-280}$ were well tolerated. However, depending on the reactions conditions, many functions may be reduced by metal-promoted hydrogen transfer $^{40,231,275}$ reactions: alkenes, carbonyl groups, nitriles, azides, halides, sulfides, and allyl ester. ${ }^{284}$ Thus, the choice of the catalyst, hydrogen source, and reactions conditions may be crucial for obtaining good chemoselectivity.

Catalyst evolution toward a "green" transfer process is a further topic of interest, ${ }^{285}$ with, for instance, (a) improvement of the handling and separation of the catalyst from the reaction products; (b) anchoring the catalyst onto a solid support, either inorganic or organic; and (c) water-soluble catalysts.

2.2.2.1. Using Zerovalent Group 10 Transition Metals. Several palladium catalysts and $\mathrm{H}$-donors have been reported.

Some mechanistic considerations were reviewed in 1985 for the heterogeneous catalytic transfer hydrogenation. ${ }^{275}$

A transfer reduction mechanism (Scheme 23) was proposed by Sato. ${ }^{278}$ It is closely related to the mechanism proposed by Trost et al. ${ }^{283}$ in 1989 using a $\mathrm{Pd}(0)-\mathrm{AcOH}-\mathrm{R}_{3} \mathrm{SiH}$ combination for alkyne and diyne reduction (Scheme 23).

a. $(\mathrm{dba})_{3} \mathrm{Pd}_{2} \cdot \mathrm{CHCl}_{3}$ or $\mathrm{Pd}\left(\mathrm{PPh}_{3}\right)_{4}$ Catalysts. In 1989, Trost $^{283}$ reported the first semihydrogenation of alkynes using a hydrogen donor in the presence of a homogeneous palladium catalyst. In the presence of $(\mathrm{dba})_{3} \mathrm{Pd}_{2} \cdot \mathrm{CHCl}_{3}(2.5$ $\mathrm{mol} \%)$ and tri-o-tolylphosphine (10 mol \%), hydrosilanes, and acetic acid as hydrogen donors in benzene, internal alkynes were quickly (60 s to $20 \mathrm{~min}$ ) reduced to $(Z)$-olefins in good yields $(65-95 \%)$, without any over-reduction or reduction of other doubles bonds in the molecule. Interestingly, esters, free hydroxyl, nitro, ODMPS, OPMB, and OTBS groups, phenylsulfides, ketone, but also isolated and conjugated double bonds did not interfere with this reduction process. ${ }^{283,286}$ Diethylphosphonate $^{287}$ and amide ${ }^{288}$ functions were well tolerated also. However, the high stereospecificity observed is dramatically spoiled, in some cases, by in an situ isomerization of the formed ( $Z$ )-double bond. ${ }^{283,287,288}(Z)$-Vinylsilanes obtained from TMS-alkynes also readily isomerized (moderate yield, $Z$ / $E$ ratio: $1 / 2$ to $1 / 3.7$; prolonged reaction times in comparison with internal alkynes were needed for full conversion).

The reaction can also be performed using hydrosilane immobilized on silica gel, showing the use of a homogeneous catalyst $\left[\mathrm{Pd}\left(\mathrm{PPh}_{3}\right)_{4}\right]$ in a heterogeneous reaction system. ${ }^{289}$

Using $(\mathrm{dba})_{3} \mathrm{Pd}_{2} \cdot \mathrm{CHCl}_{3}$ catalyst and tri( $n$-butyl)phosphine, Sato reported ${ }^{278} \mathrm{HCOOH}_{-\mathrm{NEt}_{3}}$ as an effective source of hydrogen in THF. Although these conditions ${ }^{278}$ are milder than the conditions developed by Trost, ${ }^{283}$ prolonged reaction times after completion of the reaction favor in situ isomerization.

\section{Scheme 23}

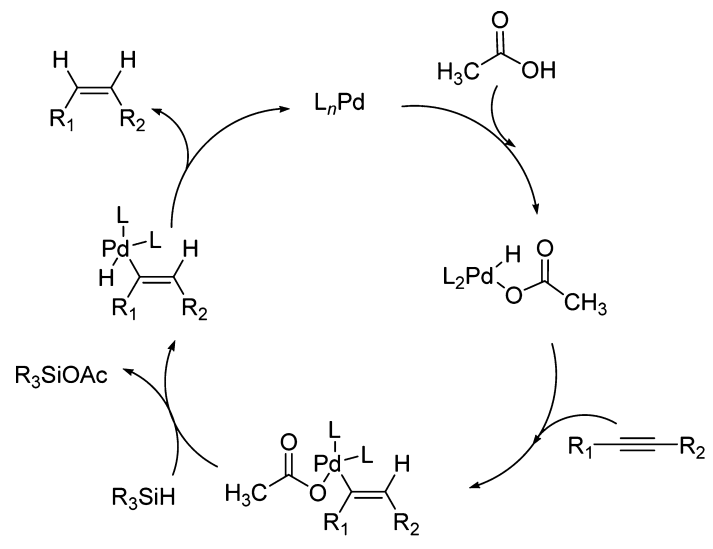

Trost's proposed mechanism

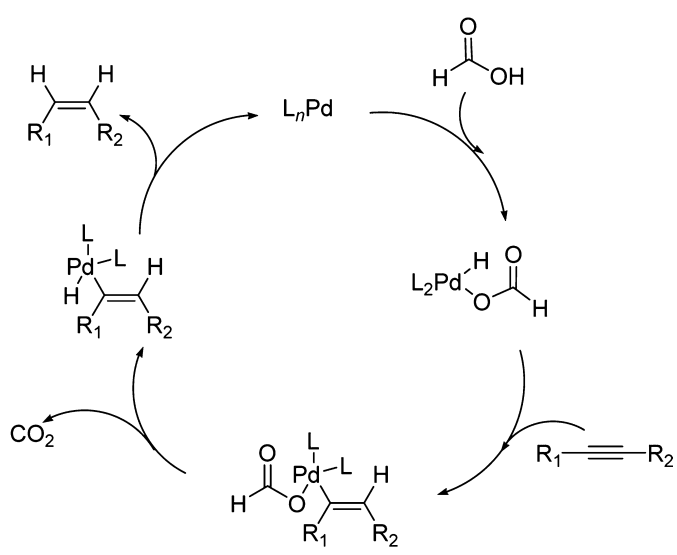

Sato's proposed mechanism 
Higher temperatures $\left(80^{\circ} \mathrm{C}\right.$ instead of $\left.40^{\circ} \mathrm{C}\right)$ were needed with $\mathrm{Pd}(\mathrm{acac})_{2} \cdot \mathrm{Bu}_{3} \mathrm{P}$ catalyst and ammonium formate $\mathrm{HCOONH}_{4}$ (4 equiv) in benzene. ${ }^{277}$

b. $P d / C$ Catalyst. Diphenylacetylene was partially reduced in moderate yield with triethylammonium formate as $\mathrm{H}$-donor, but the overhydrogenation reaction is a major issue under these conditions with many substrates. ${ }^{290}$

Recently, phenylalkynes were reduced to $(Z)$-styrene using a stoichiometric amount of Hantzsch ester 1,4-dihydropyridine as hydrogen source and $\mathrm{Pd} / \mathrm{C}$ as catalyst. ${ }^{291}$ The reactions were performed in ethanol at reflux. The Hantzsch ester 1,4dihydropyridine is commercially available, although rather expensive.

Good yields were obtained for $(Z)$-styrenes bearing electron donating groups (Scheme 24) such as methyl, methoxy, or hydroxyl groups. However, low yields were obtained in the presence of electron withdrawing groups (nitro, chloride, ...).

\section{Scheme 24}

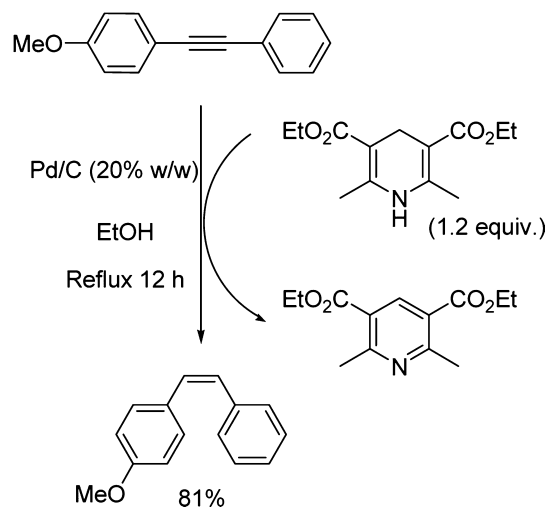

No $(E)$-regioisomer and only traces of alkanes were detected. However, only a few examples with functionalized internal alkynes were reported till now.

c. Pd(0)-Carbene Catalyst. The semihydrogenation of alkynes to $(Z)$-alkenes shows excellent chemo- and stereoselectivity when using a zerovalent palladium(NHC)(maleic anhydride) complex as precatalyst and triethylammonium formate as hydrogen donor. Thus, Hauwert et al. described $^{279,292}$ a system for semihydrogenation of alkynes that cleanly and reproducibly leads to $(Z)$-alkenes, using $\mathrm{Pd}^{0}$ (IMes)(MA) complex as precatalyst, which is prepared in situ from the standard $\mathrm{Pd}^{0}$-precursor $\mathrm{Pd}(\mathrm{tBuDAB})(\mathrm{MA}), 1,3-$ bis(mesityl)imidazolium chloride, and potassium tert-butoxide. The over-reduction to alkane is fully inhibited when formic acid is used as hydrogen donor in strongly coordinating solvents whereas the use of hydrogen gas still gives over-reduction.

In some cases, the stereoselectivity of the reaction is highly dependent on the nature of the solvent. For instance, reduction of 4-octyne in THF provides $99 \%$ of the corresponding $(E)$ alkene while the same reaction conducted in acetonitrile affords $93 \%$ of $(Z)$-alkene. ${ }^{279}$ Besides, prolonged reaction times tend to favor isomerization. Deactivation of the alkyne bond with some electron-withdrawing groups led to a poor selectivity. A novel air-stable series of $\mathrm{Pd}(0)$ complexes with heteroditopic NHCamine ligands ${ }^{293}$ or $\mathrm{N}$-pyridyl-NHC ligands, obtained by transmetalation $^{294}$ from their corresponding silver(I) complexes, also showed high stereoselectivities in semihydrogenation of alkynes.

Hauwert et al. suggested a mechanism in which a hydrogen transfer from coordinated formate anion to zerovalent palladium(NHC)(MA)(alkyne)-complex is followed by migratory insertion of hydride, after which the alkene is liberated by proton transfer from the triethylammonium cation. ${ }^{292}$ The equilibrium between precatalyst (IMes) $\mathrm{Pd}(\mathrm{MeCN})(\mathrm{MA})$ and substrates gives several potentially active species (Scheme 25). ${ }^{292}$

d. $P d(O A C)_{2}$ Catalyst. In 2003, Wei et al. reported ${ }^{282}$ the use of $\mathrm{Pd}(\mathrm{OAc})_{2}$ catalyst in the presence of sodium methanolate $(5$ equiv) and $\mathrm{PPh}_{3}(5 \mathrm{~mol} \%)$ as reductive agent. The reaction proceeds through a palladium methanolate complex followed by $\beta$-hydride elimination and reductive elimination (Scheme 26). ${ }^{282}$

This $\mathrm{Pd}(\mathrm{OAc})_{2}-\mathrm{NaOMe}$ catalytic system prepared in THF was studied with diarylalkynes, providing the corresponding alkenes in moderate to good yield with an excellent (Z)stereoselectivity. Replacement of THF by $\mathrm{MeOH}$ led, in most cases, to the fully saturated analogs.

Another variation suggested $\mathrm{DMF} / \mathrm{KOH}$ as an $\mathrm{HCOOH}$ in situ production (Scheme 27). ${ }^{295}$

This reducing system was successfully used for the synthesis of the Combrestatine A-4 analog (Scheme 28).

Furthermore, using $\mathrm{Bu}_{3} \mathrm{SnH}$ or $\mathrm{Ph}_{2} \mathrm{SiH}_{2}$ as hydride source, the reduction with $\mathrm{Pd}(\mathrm{OAc})_{2}$ catalyst in the presence of $N, N^{\prime}$ bis(benzylidene)ethylenediamine (BBEDA) and $\mathrm{AcOH}$ (2 equiv) in benzene or toluene at reflux resulted in the obtention of the (Z)-alkene, without affecting a cyclic double bond.

\section{Scheme 25}

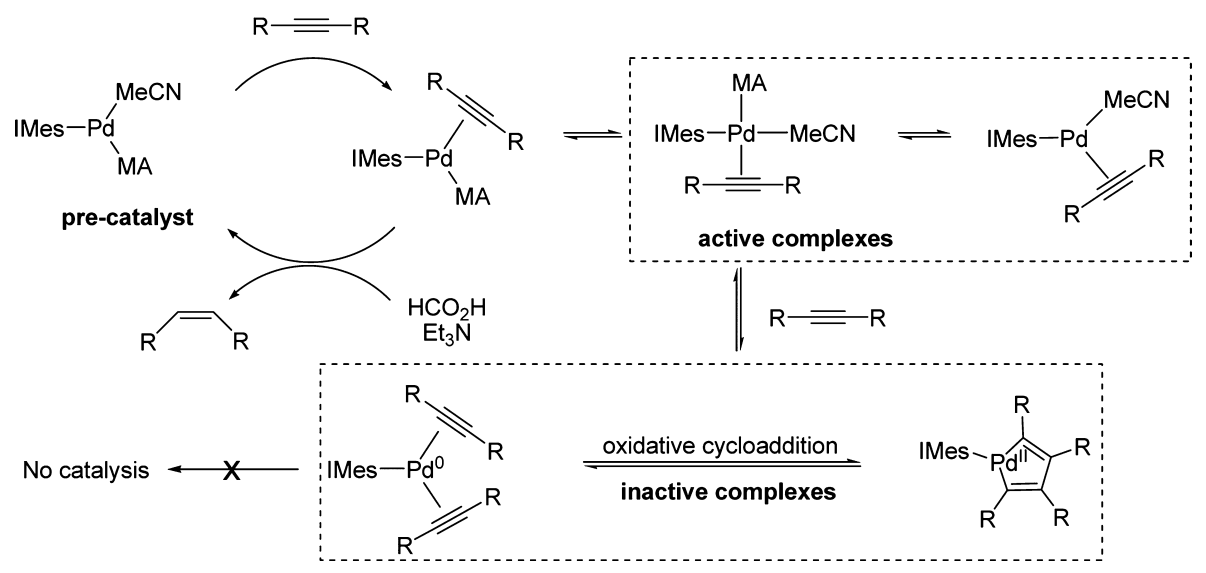


Scheme 26

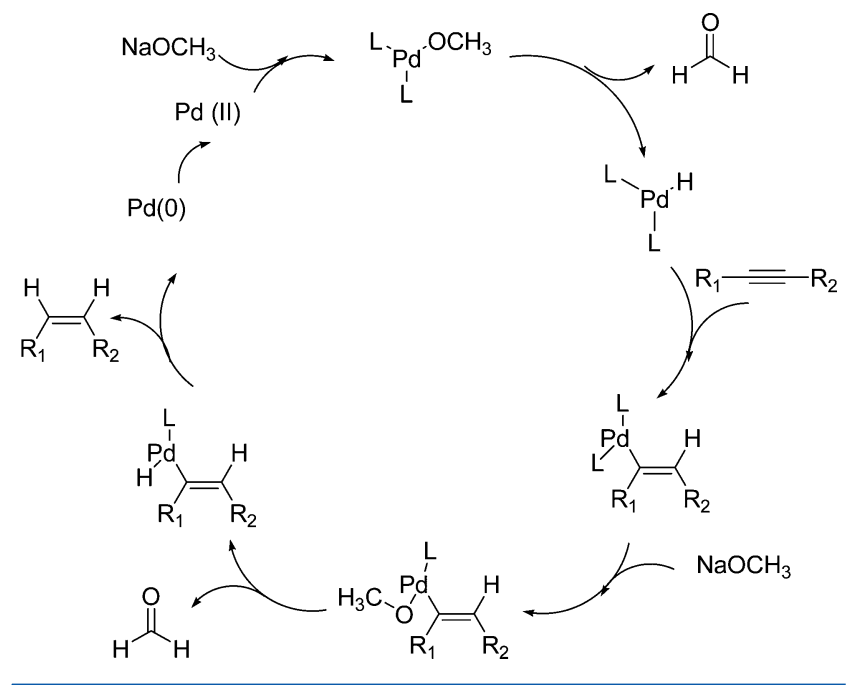

Scheme 27
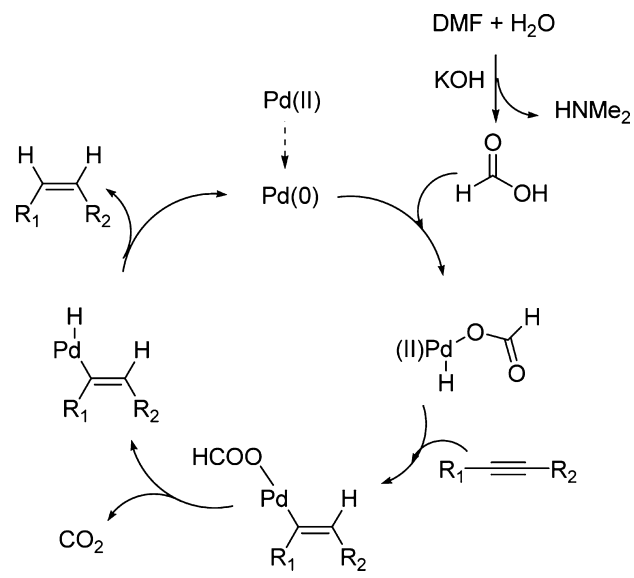

Scheme 28

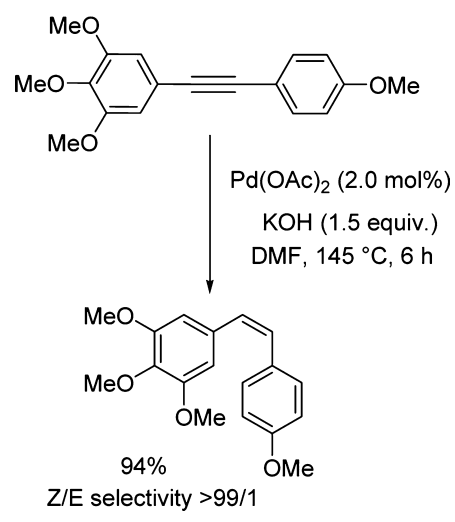

However, replacement of the hydride with PMHS favored the spontaneous competitive cyclization with the in situ formed Pdactivated olefin, leading to a bicyclic structure with an exocyclic (E)-olefin. ${ }^{296} \mathrm{NCBz}$ and free hydroxyl groups were tolerated. ${ }^{296}$

e. Other Group 10 Transition Metal Complexes. Recently, a facile regio- and stereoselective hydrometalation of alkynes was obtained on the basis of a combination of carboxylic acids and group 10 transition metal complexes $\mathrm{M}\left(\mathrm{PEt}_{3}\right)_{4}$ with $\mathrm{M}=\mathrm{Ni}$, $\mathrm{Pd}, \mathrm{Pt}^{297}$ Application to the reduction of alkynes showed that this process using formic acid as $\mathrm{H}$-donor can selectively produce the cis- or trans-alkenes or alkanes by slightly tuning the conditions (Scheme 29). ${ }^{297}$

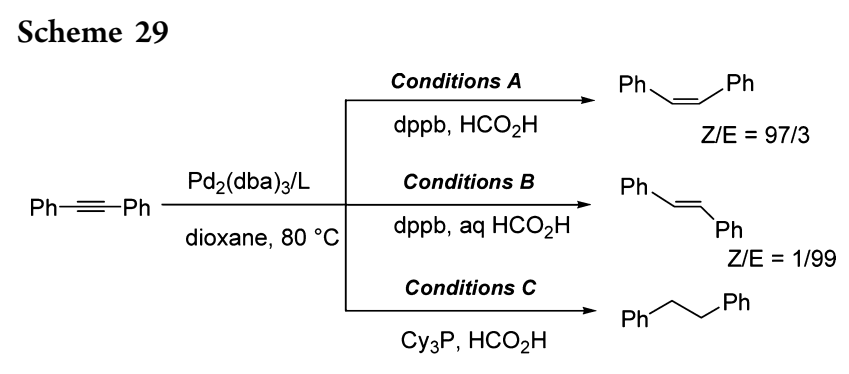

Yields and stereoselectivities are excellent. The nature of the phosphine plays a crucial role: the use of 1,4-bis(diphenylphosphino)butane (dppb) prevents over-reduction while the use of $\mathrm{Cy}_{3} \mathrm{P}$ turned alkynes to alkanes exclusively.

Most of the internal alkynes used as starting material were phenylacetylene derivatives. Except ester groups, ferrocene, phenol, and aniline, no other functional group was present in the molecules. Thus, it is difficult to predict the chemoselectivity of the reaction (dioxane, $80{ }^{\circ} \mathrm{C}, \mathrm{HCOOH} \mathrm{2-6} \mathrm{equiv,}$ 3-48 h), in the presence of other double bonds.

On the basis of previous proposed mechanisms, a catalytic cycle involving hydropalladation of the triple bond with formic acid, followed by decarboxylation and reductive elimination, is proposed to afford ( $Z$ )-alkenes (Scheme 30). ${ }^{297}$

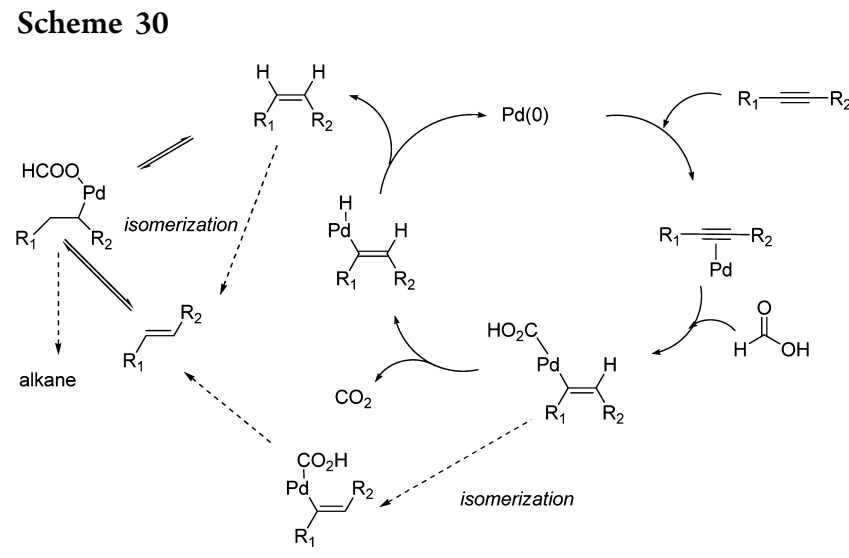

2.2.2.2. Using Ruthenium. Semireduction of alkynes using ruthenium catalysts is usually performed under hydrogen atmosphere. ${ }^{298-300}$ However, recently, Belger et al. ${ }^{298}$ reported the first ruthenium-catalyzed semireduction of alkynes to $(Z)$ alkenes under transfer hydrogenation conditions. No problem with over-reduction to the corresponding alkane (even after prolongated reaction times) and high yields (89-96\%) was observed. The stereoselectivity is dependent on the solvent, the concentration, the nature of the $\mathrm{H}$-donor, and temperature, with the best conditions being $1.25 \%$ mol $\mathrm{Ru}\left(\mathrm{PPh}_{3}\right)_{3} \mathrm{Cl}_{2}$, $\mathrm{DMF} / \mathrm{HCOOH}: 1 / 2\left(c=1.3 \mathrm{~mol} \cdot \mathrm{L}^{-1}\right)$, rt. Many functions, such as NHphtal, chlorine atoms, free hydroxyl groups, methyl esters, OBz, OTBS, and ketones, were compatible with the reductive conditions. A nitrile group, however, seriously disturbs the course of the reaction (10\% yield).

Comparison with semihydrogenation using the Lindlar catalyst showed slightly lower yields and stereoselectivities, but careful control of hydrogen gas uptake is not required to minimize the formation of over-reduced side-products. $\mathrm{Ru}$ - 
$\left(\mathrm{PPh}_{3}\right)_{3} \mathrm{Cl}_{2}$ is a convenient air-stable complex synthesized from the inexpensive $\left[\mathrm{RuCl}_{3}\right]$.

On the basis of their previous work, Belger et al. proposed a mechanism via a hydrometalation step (Scheme 31). ${ }^{298}$

\section{Scheme 31}

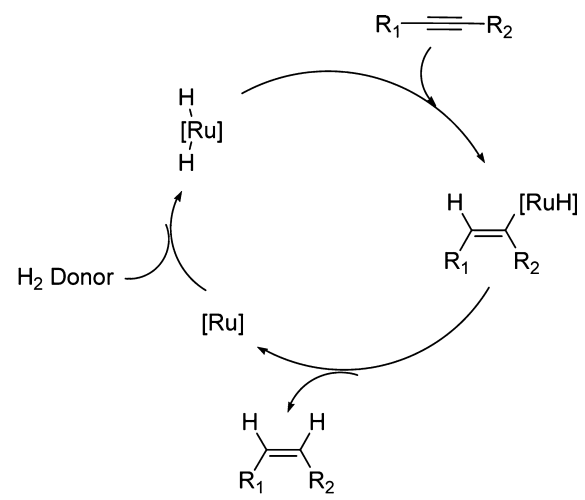

2.2.2.3. Using Diruthenium Complex. The diruthenium(0) $\left[\mathrm{Ru}_{2}(\mu-\mathrm{CO})(\mathrm{CO})_{4}(\mu-\mathrm{dppm})_{2}\right]$ complex $(\mathrm{dppm}=$ $\left.\mathrm{Ph}_{2} \mathrm{PCH}_{2} \mathrm{PPh}_{2}\right)$ is an efficient catalyst for reduction of internal alkynes by hydrogen transfer from formic acid. ${ }^{301}$ The presence of electron withdrawing groups (such as COOMe) seriously disturbs the course of the reaction.

2.2.2.4. Using Dodecacarbonyltriruthenium Complex $\left(R u_{3}(C O)_{12}\right) . \mathrm{Li}$ and Hua reported in 2011 a stereodivergent ruthenium-catalyzed transfer semihydrogenation of diaryl alkynes with DMF and water as hydrogen source affording cis- and trans-stilbenes. ${ }^{302}$ The cis or trans isomer can be selectively reached by tuning the reaction conditions using respectively acetic acid or trifluoroacetic acid as additive.

2.2.2.5. Using Titanium. In the presence of $\mathrm{Cp}_{2} \mathrm{TiCl}(2.5$ equiv), water (10 equiv) can be used as the $\mathrm{H}$-atom source for the reduction of alkynes with a substoichiometric ( 0.1 equiv) quantity of the Lindlar catalyst (moderate to good yield). ${ }^{280}$ Surprisingly, the authors did not compare the results with the hydrogenation reaction using the Lindlar catalyst and hydrogen gas.

2.2.2.6. Using Cobalt. In ethanol, the complex species prepared from cobalt(II) salt $(0.1 \mathrm{~mol} \%)$ and $\mathrm{NaBH}_{4}(0.2-0.5$ mol \%), assumed to be a cobalt hydride, readily reduced unsaturated compounds. However, even with a limited amount of $\mathrm{NaBH}_{4}$, this reductive system suffers from a bad chemoselectivity, leading to a large amount of alkanes. ${ }^{303}$

2.2.2.7. Using Iridium. Hydrido(methoxo)iridium(III) complexes serve as efficient catalysts for reduction of alkynes using methanol as a source of donor. However, they yielded the transalkenes as the major product. ${ }^{304}$

\section{APPLICATIONS IN TOTAL SYNTHESIS}

This part of the review intends to give hints for practitioners and help them to choose the most suitable reducing system for their polyfunctionalized substrate. We wondered whether the choice of the reducing system depends on the complexity of the alkyne substrate and the type and/or location of functional groups. Most importantly, we wish to highlight which reducing systems provide the best stereo-, regio-, and chemoselectivities (mainly in the presence of double bond(s)).

\subsection{The Lindlar Catalyst}

The Lindlar catalyst is widely employed in total synthesis, since the catalyst is stable and easy to handle, and good stereoselectivities were reported.

However, as we will discuss below, in many cases it happened to be more tricky and delicate than expected. In some cases, the authors gave up due to misunderstood problems of reproducibility $^{122,305}$ or inconsistent results. ${ }^{306}$ It must be stressed that the quality of the catalyst may fluctuate a lot from the different providers or from different batches from the same provider. In 1984, Adams et al. ${ }^{307}$ encountered difficulties and obtained very low yields with some substrates. They warmly advised purchase of the Lindlar catalyst from Strem Chemicals, since, in their hands, Lindlar catalysts from other providers gave worse results. It must be mentioned that Lindlar catalyst gave erratic results, as reported by Feutrill et al., ${ }^{308}$ and also Kluwer et al. $^{134}$

In addition, attention must be paid, since in the literature the 'Lindlar' term is confusing. Effectively, in some cases in the discussion section it is written "Lindlar" while the experimental part described a $\mathrm{Pd} / \mathrm{BaSO}_{4}{ }^{63,309-312}$ or $\mathrm{Pd} / \mathrm{CaSO}_{4}{ }^{150}$ reagent or the contrary. 313,64

Moreover, sometimes the authors simply quote "Lindlar" without mentioning whether they used a base additive or not. However, as discussed in section 2.1.1.1a, the nature of the reagent used to desactivate the surface of the catalyst metal, together with its quantity, are crucial for obtaining the desired target in high yield and excellent purity. In a polyfunctionalized substrate the reaction conditions are crucial.

Lindlar hydrogenation reactions were carried out in various solvents from nonpolar to protic polar. The solvents most commonly reported in the literature are benzene, ${ }^{314-318} n$ hexane, ${ }^{319-323}$ ethyl acetate, ${ }^{324,325}$ methanol ${ }^{326-331}$ or ethanol, $^{332,333}$ but also toluene, ${ }^{334}$ cyclohexane, ${ }^{335}$ heptane, ${ }^{336}$ petroleum ether, ${ }^{337}$ dichloromethane, ${ }^{338} \mathrm{Et}_{2} \mathrm{O},{ }^{339}$ EtOAc$\mathrm{EtOH}(2 / 1),{ }^{340}$ or $\mathrm{Et}_{2} \mathrm{O}-\mathrm{THF}^{341}$ or dry acetone $-\mathrm{THF}^{342}$ or a $\mathrm{CH}_{2} \mathrm{Cl}_{2}$-hexane $(1 / 1)$ mixture ${ }^{343}$ were also used.

The choice of the solvent seems based on the solubility of the substrate but also dramatically influences the course of the reaction. ${ }^{25,344}$

In polyunsaturated structures, the reactions were usually run at cold to chilly temperatures $\left(0^{\circ} \mathrm{C},{ }^{327,339,345} 5-8{ }^{\circ} \mathrm{C},{ }^{346,347} 10\right.$ ${ }^{\circ} \mathrm{C},{ }^{315-317,341,348}$ or around $\left.15^{\circ} \mathrm{C}^{349}\right)$, although some experiments were performed at $\mathrm{rt}^{320,334,350}$ A good example of the importance of the temperature is given by Kang et al. ${ }^{345}$ (Scheme 32). At $\mathrm{rt}$, the partial reduction reaction failed: only unidentified byproducts were obtained. On the contrary, the reaction proceeds in good yield (ca. 84\%) at $0{ }^{\circ} \mathrm{C}$.

The amount of palladium catalyst varies notably from one report to another. Most authors add about 5-10 wt \% catalyst, but the quantity is sometimes increased up to a $2 / 1$ : "catalyst" to substrate ratio. ${ }^{348,351}$ The experiment is sometimes stopped to be launched again with another batch of catalyst.

Most of the hydrogenation reactions over the Lindlar catalyst were carried out at atmospheric pressure.

A few examples are, however, performed under pressure in a Parr apparatus at $400 \mathrm{hPa}^{348,351}$ or $18 \mathrm{psi}^{352} 28 \mathrm{psi}^{353}$ However, these latter conditions are not appealing, since they yielded a mixture containing the desired diene together with the intermediate enyne and the starting diyne. Actually, the presence of a significant amount of under-reduced structures is all the more surprising, since the authors did not report the use of any reagent to poison the palladium surface. 
Scheme $32^{345}$

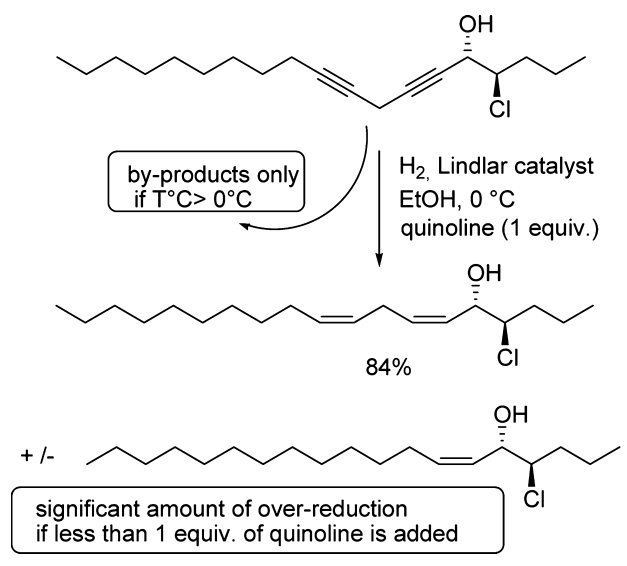

The reaction conditions are compatible with the presence of many functional groups, including methyl ester, $315,316,320,354,355$ conjugated ester, ${ }^{356,357}$ OBO ester, ${ }^{340,358}$ carboxylic acid function, ${ }^{324,359,360}$ free hydroxyl groups, ${ }^{314,315,317,319,322 \text {, }}$ $324,326,329,341$ epoxide, ${ }^{319,327,328,335,349}$ acetate, ${ }^{361}$ acetal, ${ }^{356}$ MOM, ${ }^{314}$ monomethoxytrityl, ${ }^{307} \mathrm{~N}$-tosyl, 340 $\mathrm{THP}^{318,325,343,362-364}$ benzoyl, ${ }^{351,365,366}$ and allylic benzoyl ${ }^{321}$ groups, but also sulfides, ${ }^{367}$ OTES, ${ }^{323}$ OTBDMS, ${ }^{320,335,368}$ OTBPS, ${ }^{307,332,340,343,355,361} \mathrm{PMB},{ }^{356,369}$ quinone moiety, ${ }^{324}$ chlorine atom, ${ }^{345}$ and 1,3-dioxolane, ${ }^{342} 1,2$-dioxolane, ${ }^{370}$ or 1,2-dithiolanes, ${ }^{360}$ thiazolidin-2-one $1,{ }^{356}$ and cyclopropane. ${ }^{371}$ The presence of $\eta^{5}$-1-substituted-pentadienyl(tricarbonyl)iron cations does not disturb the course of the reaction (99\% yield starting from a diyne). ${ }^{352}$

The Lindlar catalyst was successfully employed for the partial

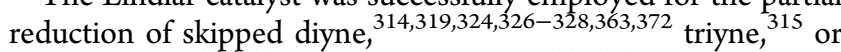
skipped triyne $e^{322,329}$ or skipped tetrayne. ${ }^{317,341}$

Thus, application to the synthesis of natural products was widely reported: for instance, pheromones (identification in female gametes of Analipus japonicus), ${ }^{329}$ PUFAs, ${ }^{373}$ hepoxilins, ${ }^{349}$ leukotriene $\mathrm{B5},{ }^{374}$ or Laurencenyne ${ }^{350}$ in the red alga Laurencia okamurai.

An example of the excellent selectivity of the partial hydrogenation of skipped tetrayne systems with the Lindlar catalyst is outlined in Scheme $33 .{ }^{339}$

\section{Scheme $33^{339}$}

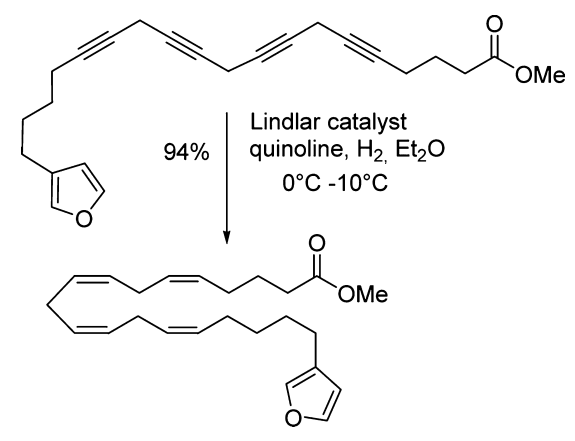

As advised by Lindlar, quinoline is usually added into the reaction mixture. ${ }^{37}$ However, its quantity is often omitted. ${ }^{325}$ It is however noteworthy that the rate and the chemoselectivity of the reaction, and thus the purity of the desired target, are strongly related to percentage of quinoline. In 2007, Kang et al. $^{345}$ pointed out (Scheme 32 ) that the use of less than one equivalent of quinoline led to partial hydrogenation of one of the two skipped alkenes in their chlorohydrin structures. In the absence of quinoline, alkenes are generally reduced to alkane in short reaction times. ${ }^{375}$

In contrast with the above-mentioned Kang's comments, ${ }^{345}$ Adlof et al. ${ }^{337}$ were surprised with the extremely slow reactivity of their diyne (flanked by a deuterated alkyl chain and a $\mathrm{CH}_{2} \mathrm{OH}$ group) in the presence of quinoline in petroleum ether. Even when no quinoline was used, an almost one to one ratio (w/w) of "catalyst" to substrate was necessary to reduce the diynol ( $96 \%$ yield with $1 \%$ of starting material and only $2.5 \%$ of further reduced material). Actually, unless omitted, it seems that many hydrogenation reactions of skipped diynes ${ }^{334,340,352,364}$ or skipped triynes ${ }^{322,329,368,370}$ were run with good to excellent yields without quinoline. Thus, it seems rather difficult to rationalize such opposite reaction requirements.

Some authors replaced quinoline with triethylamine $\mathrm{e}^{320,321,346}$ or pyridine ${ }^{323}$ (with different ranges of amount $=1 / 10,000,{ }^{376}$ $1 \%$ pyridine $\left.^{307}\right)$. Lellouche et al. $^{377,378}$ observed that the reaction mixture (hexane) must contain exactly $0.01 \%$ pyridine in volume. An excess of pyridine (0.02-0.05\%) inhibits hydrogen or deuterium absorption.

In 2005, instead of amines, Nakanishi added an excess of cyclohexene to prevent over-reduction of a skipped dieneepoxide. $^{335}$ Khrimian et al. ${ }^{328}$ also successfully used cyclohexene for chemoselective preparation (93\% yield, 95\% purity) of $(Z, Z)$-octadienyl-3-nonyloxirane, a pheromone of Pink Moth, Lymantria mathura.

Similarly, in 2007, Naidu et al. ${ }^{44}$ came against serious difficulties with "standard" conditions (application to a procedure reported by Nicolaou ${ }^{379}$ or Overman $\left.^{369}\right)$. The partial hydrogenation of a triple bond, conjugated to an $(E)$ olefin yielded the desired $(Z, E)$-diene, but it was systematically contaminated with overhydrogenated products whatever the quantities of quinoline (from substoichiometric quantities to several molar equivalents). The addition of 1-octene as a cosolvent along with EtOAc in the presence of pyridine (EtOAc/1-octene/pyridine: 10/1/1) nicely improved the course of the reaction, providing the expected pure conjugated $(Z, E)$-diene in $95 \%$ yield (Scheme 34$)$.

3.1.1. Chemoselectivity. Scheme 35 highlights the high chemoselectivity of the hydrogenation over Lindlar catalyst in the total synthesis of $7(S), 17(S)$-resolvin D5, a potent antiinflammatory docosanoid: Rodriguez et al. ${ }^{321}$ nicely reduced a skipped diyne in a structure which also contained four other double bonds (two (E)-allylic double bonds and two isolated (Z)-double bonds). However, this elegant result may not be easily reproduced, since the authors did not specify the exact reaction conditions.

Besides ( $Z$ )- or (E)-allylic functions, ${ }^{314,321,379,380}$ cyclic double bond, ${ }^{336}$ isolated $(E)-^{361,381}$ or $(Z){ }_{-}{ }^{332,356}$ double bond, conjugated $(E)$-olefin, ${ }^{333,357,372}$ or a terminal alkene, ${ }^{314}$ the semireduction of isolated alkynes and/or poly skipped diynes also showed a high chemoselectivity in the presence of $(Z, Z)$-bis-allylic systems, ${ }^{361,379}$ but it is also respectful of conjugated 1,3 -diene $\left((E, Z)\right.$-diene $e^{307,320,360}$ or $(E, E)$ dien $^{338,378}$ ) and conjugated (E,E,E)-triene. ${ }^{331}$ Thus, this methodology is widely used in the synthesis of natural products such as leukotriene B4, ${ }^{355,382}$ HETEs, ${ }^{199,323}$ lipoxins, ${ }^{383-385} 11$ hydroxy linoleic acid, ${ }^{386}$ and related eicosanoids, ${ }^{385}$ but also cyclic PUFA metabolites such as $15-\mathrm{E}_{2}$-isoprostane ${ }^{387}$ or 
Scheme $34^{44}$

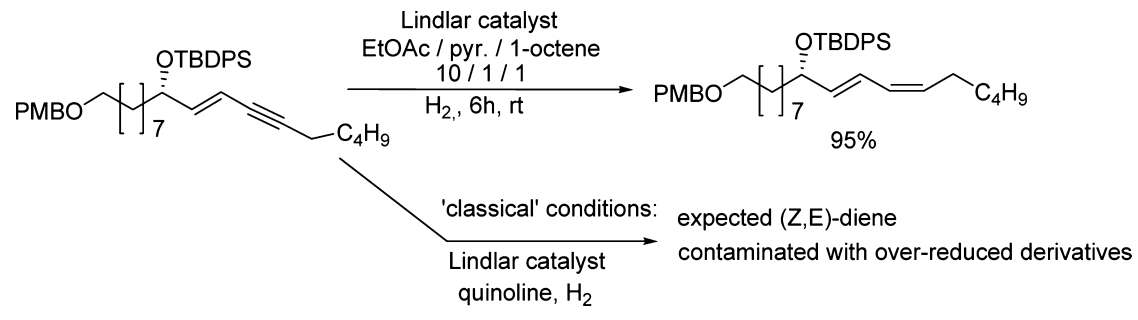

Scheme $35^{321}$
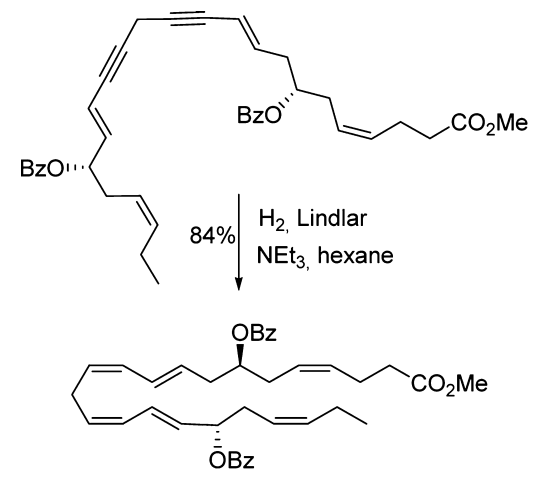

pheromones (identification in female gametes of Analipus japonicus), ${ }^{329}$ carotenoids. $^{388,389}$

An enone function is however affected. The hydrogenation of a skipped diyne system in a dienenone containing molecule was complicated by a competitive over-reduction of one and/or both double bonds of the dienone moiety. The double bonds survived the hydrogenation conditions if the ketone function is protected as a 1,2-dithiolane. ${ }^{360}$ In addition, Corey admitted that the Lindlar reduction of an epoxy-1,4-diyne was difficult to control. ${ }^{390}$

3.1.2. Regioselectivity-The Case of TMS-Protected Diyne. It appeared that protection of a terminal ethynyl group with trimethylsilyl makes the alkyne less reactive to Lindlar hydrogenation and permits high regio- and chemoselective reduction of internal triple bonds.

Application of this strategy to the total synthesis of a potent ant toxin is depicted in Scheme $36 .{ }^{391}$ The alkyne which is protected by the trimetylsilyl group is not reduced and is not deprotected. In addition, the $(Z)$-double bond of the starting material remained unchanged.

This process offers an elegant method to synthesize terminal alkynes substituted with polyunsaturated chains in a highly specific and stereoselective manner (and good yields) as useful precursors for Sonogashira or Suzuki cross-coupling reactions. This high selectivity was highlighted in the total synthesis of

Scheme $36^{391}$ several natural products, such as 12 -HETE, ${ }^{379}$ methyl $(5 Z, 8 Z, 10 E, 12 E, 14 Z)$-eicosapentaenoate, ${ }^{330}$ a trioxilin A3 precursor, ${ }^{392}$ and preclavulone A analogs, ${ }^{393}$ for instance.

Likewise, starting from a closely related TMS-diyne-ene substrate, Rowley et al. ${ }^{336}$ obtained a similarly good chemo-, regio-, and stereoselectivity. They observed that the semireduction of the protected terminal acetylene function also occurred to a varying extent from reaction to reaction. The triene side product was easily removed after desilylation of the terminal alkyne.

Due to different local environments, some triple bonds in poly yne structures may show important differences in the rate of their reactivity, leading to the expected fully semihydrogenated derivative together with some nonyet-hydrogenated alkynes.

For instance, despite six reloadings of the catalyst, the hydrogenation reaction with a 5,8,14-triyne could not go to completion $^{394}$ (Scheme 37). The expected triene is obtained in $37 \%$ yield only together with a nearly equal amount (34\%) of a diene-yne analog. However, once isolated and using the same hydrogenation conditions, this diene-yne intermediate could readily be converted to the desired triene ( $87 \%$ ). In this $5,8,14-$ triyne, the ease of hydrogenation of the triple bonds decreased in the order $\mathrm{C} 5-\mathrm{C} 6>\mathrm{C} 8-\mathrm{C} 9 \gg \mathrm{C} 14-\mathrm{C} 15$. This was explained by a hairpin conformation of the molecule. ${ }^{395}$

In 2000, Lapitskaya et al. ${ }^{395}$ also showed evidence of the reactivity of some alkene functions in the presence of Lindlar catalyst. They played with the difference of reactivity of the three olefinic functions in order to obtain the methyl ester trioxilins TrXB2. Thus, while the C14-C15 double bond is unaffected by the hydrogenation reaction conditions (benzene containing some pyridine $(0.2 \mathrm{v} / \mathrm{v})$ at $20{ }^{\circ} \mathrm{C}$ for $\left.5 \mathrm{~h}\right)$, the $\mathrm{C} 5-$ C6 or the C8-C9 double bond, or both of them, is reduced to alkane, providing a complex mixture.

Even without forcing up the conditions so far, some alkyne functions are sometimes not yet reduced to alkenes while some others are over-reduced to alkanes, leading to complex mixtures which are difficult to analyze and purify. Indeed, the control of the selectivity strongly depends on several factors: the percentage of quinoline, the amount of palladium (and its quality), the easy access to the surface of the palladium, the presence of some other double bonds, and competition with the access.

Thus, depending on the local environment of the alkynes and alkenes on the structure, but also the amount of quinoline and palladium catalyst introduced in the reaction mixture, the Lindlar hydrogenation conditions may result in the same sample in both over-reduced and under-reduced derivatives. Thus, the reactivity of alkynes or poly ynes in molecules containing other olefins is hard to foresee, and reaction conditions may be rather difficult to optimize. 
Scheme $37^{394}$

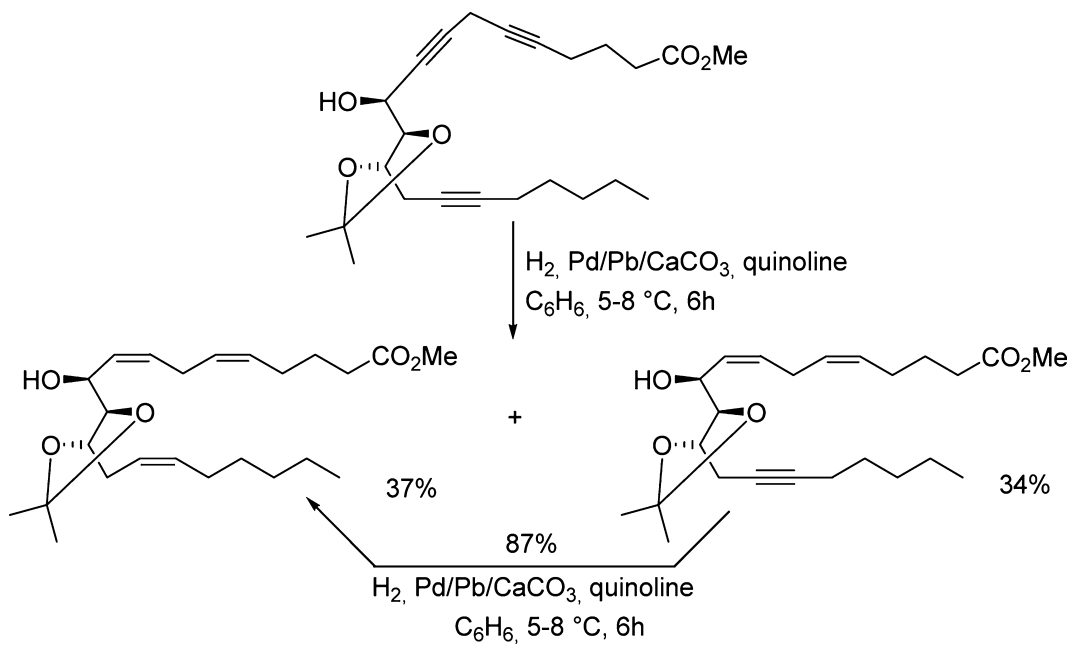

Scheme $38^{307}$
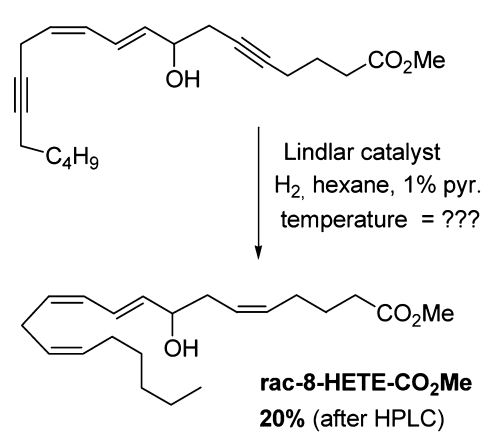

$+30-40 \%$ over-reduction
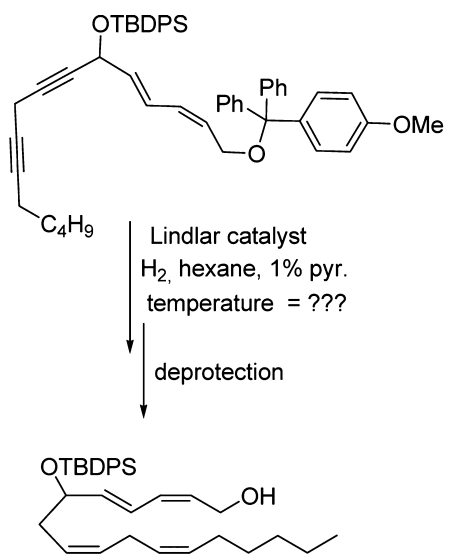

$80 \%$ (pure and isolated)

$+/$ - over-reduction less than $\mathbf{5 \%}$

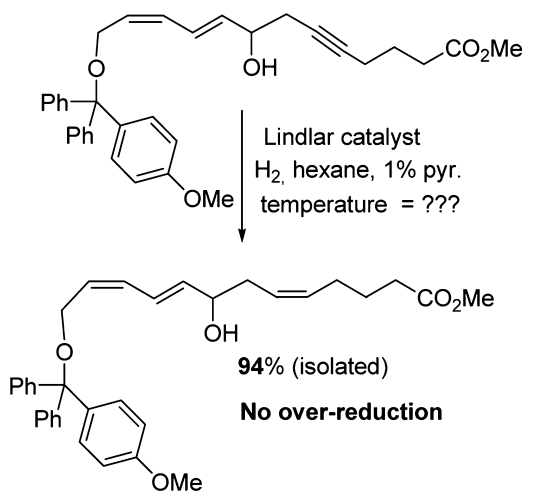

Scheme $39^{366,354, a}$
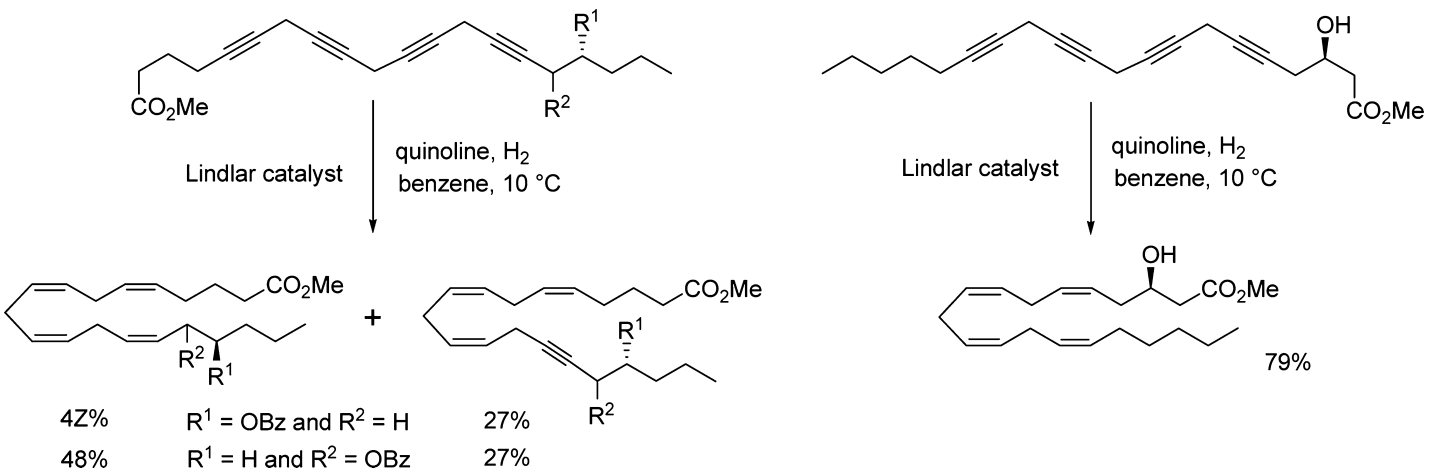

${ }^{a}$ Yields given after RP-HPLC.

Unfortunately, in some cases, the presence of byproducts, even in a small amount, dramatically hampered the purification step, lowering significantly the yield of the isolated pure compound. ${ }^{354,360}$

Gronquist et al. reported that the presence of about $15 \%$ of over-reduction products prevented the isolation of the pure expected 1,3-diene. ${ }^{340}$ Preparative HPLC is often required, even if the hydrogenation reaction proved to be nicely efficient. $316,338,347,354,365$

In addition, some authors also suggest to remove traces of over-reduced and under-reduced products by argentation chromatography ${ }^{396}\left(10-20 \% \mathrm{AgNO}_{3}\right.$ on silica gel). ${ }^{325,346,397}$

Below, a few examples illustrate the difficulty in controlling the selectivity of the hydrogenation reaction over Lindlar catalyst. 
Adams et al. ${ }^{307}$ suggested that steric hindrance may protect the double bond from reduction. Effectively, whereas a $(Z, E)$ diene function was competitively reduced (up to $30-40 \%$ ) along with alkyne functions in a diynic rac-8-HETE precursor, some other $(Z, E)$-dienes were not affected when they were surrounded by cumbersome groups.

Scheme 38 summarizes these results: despite many efforts, the desired methyl ester of rac-8-HETE could be obtained in $20 \%$ yield only. By contrast, the bulky methoxytrityl and silyl groups flanking both sides of the $(Z, E)$-diene may mask it from the catalyst surface and prevent over-reduction.

Similarly, in a skipped tetrayne structure (Scheme 39) the close neighborhood of a benzoate residue to an acetylene moiety impacted its hydrogenation efficiency, due to a steric hindrance that bothers the accessibility for the palladium catalyst. ${ }^{317,354}$ Hence, the hindered alkyne function reacted very slowly only while the three other triple bonds of the starting skipped tetrayne were readily semihydrogenated. By contrast, starting from a closely related skipped tetrayne in which the homopropargylic alcohol function was not protected, the expected skipped tetraene derivative was isolated in a much better yield (79\% instead of 47\%, Scheme 39).

As mentioned previously in this review, the Lindlar hydrogenation reaction is highly stereoselective and the presence of other olefins in the molecule is usually welltolerated.

However, in some base-sensitive systems, an unexpected isomerization of $(Z)$-double bonds was observed. For instance, Batista-Pereira et al. ${ }^{398}$ could synthesize a $(3 Z, 6 Z, 8 E)$ dodecatriene, the major component of sex pheromones of some alates, in good yield ( $70 \%$ yield, Scheme 40$)$ via a $(8 Z)$ -

\section{Scheme 40}

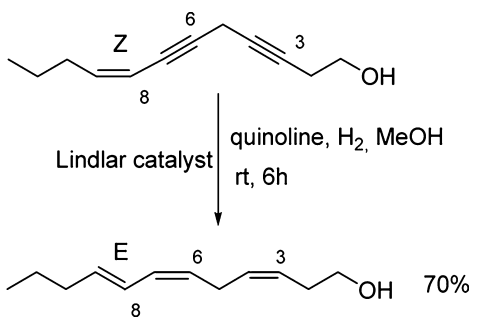

diynic precursor. The hydrogenation of the skipped diyne at the C3-C6 position furnished the expected skipped $(Z, Z)$-diene, but the resulted conjugated $(6 Z, 8 Z)$-diene readily rearranged to the more stable $(6 Z, 8 E)$-diene.

Scheme 40 also pointed out that some $(Z)$-allylic double bonds underwent cis-trans-isomerization in a significant amount (11\%) when Lindlar palladium catalyst is used under some harsh conditions, such as $0.2 \mathrm{v} / \mathrm{v}$ of pyridine at rt (overreduction desired in that case). ${ }^{394}$ On basis of examples ${ }^{58}$ using $\mathrm{Pd} / \mathrm{C}$, the authors concluded $^{395}$ that the extent of this isomerization is unpredictable and depends on the origin of the catalyst.

In the synthesis of lipoxin A4, Nicolaou et al. also reported ${ }^{338}$ the isomerization of the desired $(7 E, 9 E, 11 Z, 13 E)$-tetraene (flanked by two hydroxyl groups) to its more stable all-trans isomer during the hydrogenation over Lindlar catalyst $\left(\mathrm{CH}_{2} \mathrm{Cl}_{2}\right.$, $\mathrm{rt}$, quinoline). The "small" amount of this isomer is not quantified but hampered the purification of the target tetraene. Reversed phase HPLC was needed to provide a pure sample (53\% yield, Scheme 41 ).
Scheme $41^{338}$

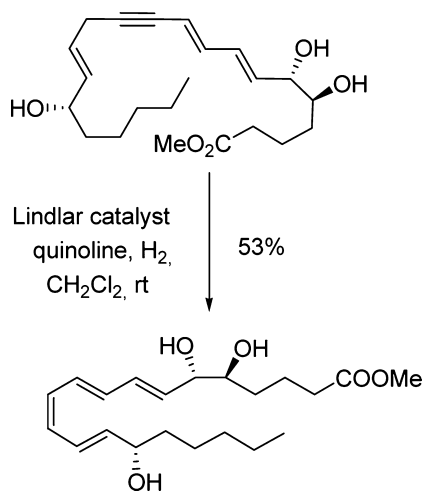

Lindlar hydrogenation conditions also converted many 1,3diynes into their corresponding conjugated $(Z, Z)$-dienes in poor to good yields. ${ }^{399-401}$ This strategy was applied to the synthesis of hexadecadienoic acid as tracer in the key step of the sex pheromone biosynthesis of the processionary moth. ${ }^{402}$ In addition, $\alpha, \beta$-unsaturated enamides were obtained, although traces of over-reduced byproducts could not be avoided. ${ }^{399}$

3.1.3. Application to the Preparation of Labeled Polyenes. The availability of deuterium and tritium-labeled polyunsaturated derivatives is essential for further investigation of their biological role. Deuterated compounds are useful internal standards in mass spectrometry analysis whereas tritium-labeled analogs facilitate studies of their metabolism and binding to receptors.

Labeled compounds were obtained by replacement of hydrogen gas with deuterium or tritium gas. Besides the problem of chemoselectivity, i.e optimization to avoid overreduction reactions, the main difficulty often stems from the unstability of the target polyenes. A HPLC purification is required to afford a high purity sample for biological assays, or quantification by mass spectrometry. Thus, the yields of the semihydrogenation reaction step are seldom given.

Skipped diyne systems were used as precursors for the incorporation of four tritium or deuterium atoms in the synthesis of labeled 5-oxo-ETEs. ${ }^{360}$ Application to the preparation of chemically sensitive structures such as leukotriene $\mathrm{d}_{4}$-LTA4 was also reported (Scheme 42 ). ${ }^{376-378}$ Over-reduction and decomposition of the epoxydiene during the purification step dramatically lowered the yield of the semireduction step.

Likewise, starting from a skipped triyne, $d_{6}$-linolenic acid was synthezised to investigate the biosynthesis of $\delta$-jasmin lactone in the yeast Sporobolomyces odorus using GC-MS method. ${ }^{359} \mathrm{~A}$ $d_{6}$-deuterated tetraenoic C19 fatty acid was prepared (>95\%

Scheme $42^{378}$

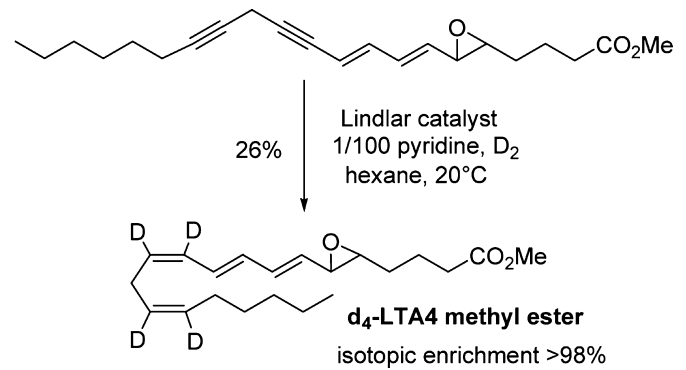


${ }^{2} \mathrm{H}_{6}, \quad 65 \%$ yield) for investigation on the biosynthesis of pheromones in female gametes of marine brown algae. ${ }^{342}$

Application of this process to tetrayne precursors led to the successful synthesis of 3-hydroxyoxylipins with tritium on all the double bonds, i.e $\left[{ }^{3} \mathrm{H}_{8}\right]-3(R)$-HETE (64\% after saponification) and $\left[{ }^{3} \mathrm{H}_{8}\right]-3(R), 18(R / S)$-DiHETE (56\%): the final tritiated products were obtained with specific activities ranging from 1.65 to $1.80 \mathrm{Ci} / \mathrm{mmol}^{351}$ The C19-hydroxy $\left[{ }^{3} \mathrm{H}_{8}\right]$ arachidonic acid analog was obtained in a high specific radioactivity $(160 \mathrm{Ci} / \mathrm{mmol})$ and a radiochemical purity of more than $95 \%{ }^{348}$ Similarly, the synthesis of labeled ${ }^{2} \mathrm{H}$ arachidonic acid was reported (no yield mentioned). ${ }^{403}$

In addition, a chemoselective reduction of one alkyne in the presence of a $(Z, E, E)$-triene flanked by two hydroxyl groups provided leukotriene B4 and its 20-hydroxy-analog with a specific activity of approximatively $40 \mathrm{Ci} / \mathrm{mmol}$. This procedure allowed their commercialization. ${ }^{404}$ Several other examples of tritium-labeled fatty acids and their oxylipin metabolites were reviewed by Shevchenko et al. in $1999 .^{405}$

\subsection{The Rosenmund Catayst}

The Rosenmund reducing system is often a good alternative to the hydrogenation over the Lindlar catalyst.

The hydrogenation reactions over $\mathrm{Pd} / \mathrm{BaSO}_{4}$ catalyst are generally run at $\mathrm{rt}$ at atmospheric pressure, and in methanol ${ }^{406-410}$ or ethanol. ${ }^{411}$ Some reactions were performed in ethyl acetate ${ }^{311,412-414}$ or nonpolar solvents such as pentane, ${ }^{313,415}$ hexane, ${ }^{309}$ or an ethyl acetate/hexane (1/1 v/ v) mixture. ${ }^{312}$ The successful hydrogenation of diyne and poly ynes was all carried out with addition of quinoline as palladium surface poison. In 1989, Saniere et al. ${ }^{414}$ had checked that, in the absence of quinoline, the hydrogenation led to the desired semireduced target in a very poor yield only.

Ketone $^{69,72}$ and epoxide ${ }^{48,65,415}$ functions, $\mathrm{THP}^{416,417}$ or $\mathrm{EE}^{412}$ groups, methyl esters, ${ }^{406,409,410,418,419}$ benzoate, ${ }^{414}$ acetate $^{65}$ acetonide, ${ }^{312,413,414}$ trityl, $^{312}$ or silylated protection such as OTBDMS, ${ }^{65,70,308}$ but also the terminal alkene ${ }^{407}$ moiety and vinylsilane group ${ }^{420}$ are not affected by the reaction conditions. The presence of a secondary amine, ${ }^{70}$ or free hydroxyl functions ${ }^{308,406,408,410,416,417}$ or a fluorine atom, ${ }^{417}$ is well tolerated.

The $\mathrm{Pd} / \mathrm{BaSO}_{4}$ catalyst was successfully employed for the partial reduction of skipped diyne, ${ }^{415,418}$ triyne, ${ }^{408,410,421}$ or tetrayne. $^{419}$

In addition, in the course of investigation on the production of defensive metabolites in Marine Diaton Skeletonema costatum), application of the $\mathrm{Pd} / \mathrm{BaSO}_{4}$ process with deuterium gas instead of hydrogen gas led to deuterated $(6 Z, 9 Z, 12 Z)$ hexadecatrienoic acid with a good yield $\left(77 \%, d_{6} 91 \%\right) .{ }^{409}$

Besides these good to excellent yields, many reactions proceeded with moderate yields only. The difficulty does not seem to be dependent on the number of triple bonds, since high yields were obtained from tetraynes ${ }^{419}$ and moderate yields from diynes ${ }^{406,407,414}$ and vice versa. ${ }^{413,418}$ The authors seldom discussed their results on that hydrogenation step in their total synthesis. It is however noteworthy that moderate yields probably mean a poor selectivity. Effectively, in several cases (diynes, ${ }^{417}$ triyne $^{311}$ ), a purification by column chromatography over $20 \% \mathrm{AgNO}_{3}$-silica gel was required to remove over-reduced or under-reduced products.

Scheme $43^{412}$ highlights the influence of the other functionalities present in the molecule on the success of the hydrogenation reaction. Mori et al. explained that the poor

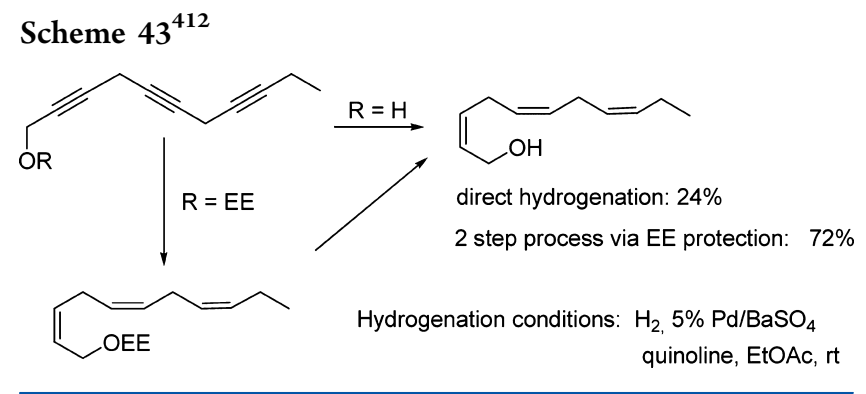

yield (24\%) obtained with the free alcohol is due to the instability of the starting triynol.

On the contrary, Scheme 44 shows the excellent stereo- and chemoselectivity of the hydrogenation reaction of a non-

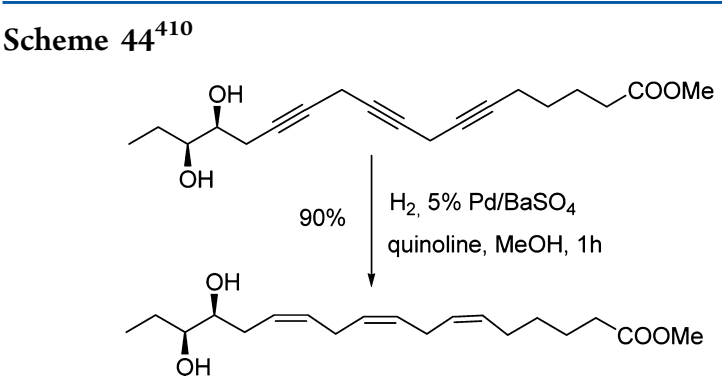

protected triyne-diol (90\% yield). ${ }^{410}$ Thus, comparison of the experiments performed in both Schemes 43 and 44 underlines the difficulty to predict the efficiency and selectivity of the semireduction of poly ynes.

Scheme 45 shows an example of moderate chemoselectivity in the presence of other double bonds. ${ }^{65}$

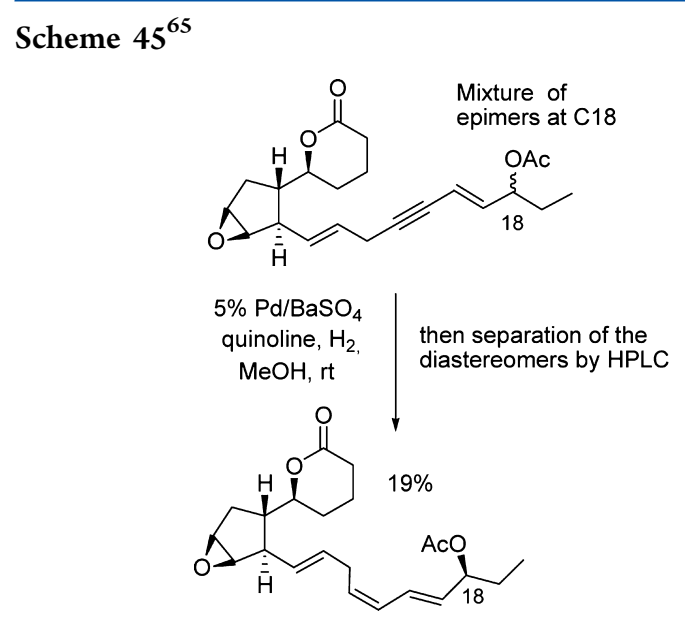

A good chemoselectivity was obtained in the presence of a conjugated $(E, E)$-diene and one isolated $(Z)$-double bond, providing the silylated leukotriene B4 in good yield (88\%). However, the ratio of quinoline to catalyst as well as the solvent system was crucial to ensure a high yield and avoid overreduction. ${ }^{344}$

3.2.1. Regioselectivity-The Case of TMS-Protected Diynes. Terminal alkynes which are protected with a trimethylsilyl group are readily reduced to trimethylsilyl alkenes. $^{70,72,312}$ Thus, by contrast with the Lindlar hydrogenation, the procedure using the $\mathrm{Pd} / \mathrm{BaSO}_{4}$ catalyst is not regioselective. 
In addition, another explanation for not using such a catalytic system probably stems from its poor stereoselectivity $(Z / E$ ratio between $4 / 1$ to $20 / 1) .^{70,72}$ Interestingly, the $Z / E$ selectivity of the reduction was found to be both concentration and protective group dependent. Moreover, in some cases, in the absence of quinoline, the TMS-alkyne is fully reduced to alkane. $^{422}$

However, very recently, in the course of the total synthesis of the protectin D1, Kobayashi selectively reduced an isolated internal alkyne in excellent yield (94\%, EtOAc, no base) without affecting a TMS-protected $(Z)$-enyne. ${ }^{423}$ In their hands, $\mathrm{Pd} / \mathrm{BaSO}_{4}$ desactivated with quinoline and Lindlar catalysts (one batch which was yet efficient in another step of their synthesis) were found to proceed quite slowly.

3.2.2. Application to the Preparation of Polyenes. Many natural products were prepared via a semihydrogenation reaction using the $\mathrm{Pd} / \mathrm{BaSO}_{4}$ system. ${ }^{25}$ For instance, Karrer et al. reported the synthesis of $\beta$-carotene ${ }^{424}$ and lycopins ${ }^{425}$ (Scheme 46$)$ in the 1950 s. Smith et al. ${ }^{426}$ accomplished a total

\section{Scheme 46}

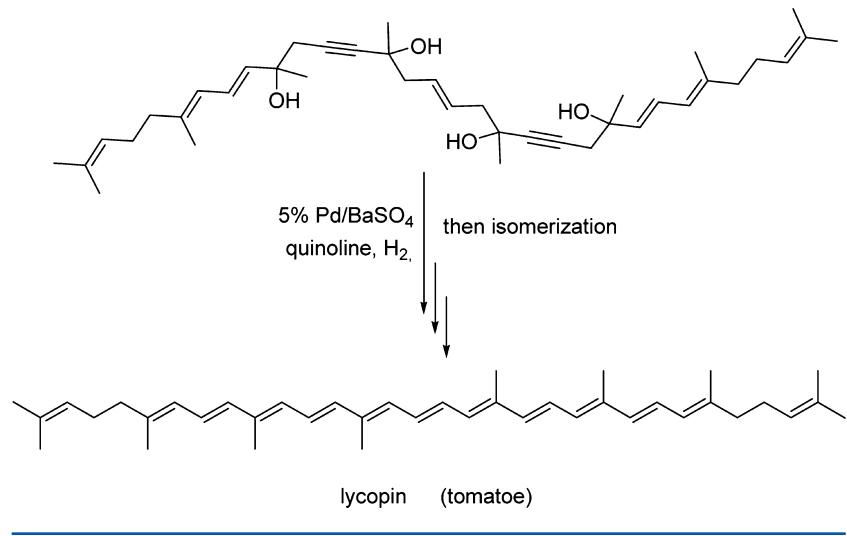

synthesis of (+)-hydroxyjatrophone A, an antileukemic diterpene macrocycle. More recently, the chemoselective hydrogenation reaction using the $\mathrm{Pd} / \mathrm{BaSO}_{4}$ system was a key step in the total synthesis of phenolic lipids, ${ }^{411}$ gemdifluoromethylenated analogs 427 of pironetin (an immunosuppressive activity), and resolvin E2 (an endogenous antiinflammatory lipid). ${ }^{428}$

Furthermore, starting from 1,3-diynes, the Rosenmund catalyst provided an efficient route to conjugated $(Z, Z)$-diene (96\% yield). ${ }^{429}$

The partial and chemoselective hydrogenation was also performed with polyfunctionalized $(Z)$-enyne on a solublepolymer support in the course of the total synthesis of the prostaglandin $\mathrm{E}_{2}$ methyl ester. ${ }^{66}$

\subsection{The Brown Catalyst}

In multistep synthesis, $\mathrm{P} 2-\mathrm{Ni}$ catalyst, also called the Brown catalyst, is probably the second most employed reducing reagent for semihydrogenation of alkyne, behind the Lindlar catalyst.

The reaction is usually run at $20{ }^{\circ} \mathrm{C}$, but cooler temperatures ${ }^{430,431}$ are also largely observed, which can account for the cutback of side products, mainly in highly functionalized molecules containing several other double bonds. The range of reaction times is relatively broad, from one hour to overnight, with the best being to monitor the hydrogen uptake. The reaction is typically run in ethanol, but examples in methanol ${ }^{118,432,433}$ were reported with no explanation for the reason behind it. The reaction conditions can be optimized by playing with the ratio of reagents. From a typical $1 / 1 / 2 / 10$ $\mathrm{Ni}(\mathrm{OAc})_{2} \cdot 4 \mathrm{H}_{2} \mathrm{O} / \mathrm{NaBH}_{4} /$ eda/alkyne ratio, the amount of eda may be increased to minimize the amount of over-reducted products. $^{430}$

The use of the Brown catalyst is compatible with almost all functionalities one chemist may think of, from free hydroxyl group, ${ }^{43-436}$ acid, $^{437}$ ester, ${ }^{119}$ acetal, $^{438-440}$ OTBDPS, ${ }^{441}$ OTBDMS, ${ }^{442}$ benzyl ether, ${ }^{443}$ propargylsilane into allylsilane, ${ }^{444}$ epoxide, ${ }^{434,445,446}$ vinyl bromide, ${ }^{328}$ vinyl ether, ${ }^{447}$ alkyl bromide, ${ }^{119,448}$ alkyl chloride, ${ }^{449}$ aromatic halides, ${ }^{450}$ pyridine derivatives, ${ }^{451}$ amine, ${ }^{450}$ to NHBOC. ${ }^{452}$ The dithiane protective group led to the formation of $2-5 \%$ of transcompound due to a free radical-induced isomerization from hydrogenolysis of the sulfur-carbone bond during the hydrogenation reaction (addition of 4-hydroxy TEMPO reduced substantially the amount of trans-isomer formed). ${ }^{453}$ A tosyl group did not show such a problem. ${ }^{454}$

Since Brown's publication in 1973 showing the potential in the semihydrogenation of alkynes, natural product chemists emphasized the effectiveness of $\mathrm{P} 2-\mathrm{Ni}$ catalyst for the hydrogenation of some very complex molecules. Nowadays it is widely used for achieving ${ }^{120}$ high stereo-, chemo-, and regioselectivity. Thus, as for the Lindlar catalyst, the Brown system has shown its appealing potential for the partial reduction of skipped dienes, ${ }^{117-119,434,438,443,455-460}$ trienes, $^{435,436,440,461-466}$ and tetraenes, ${ }^{430,467}$ mostly for PUFAs $^{124,436,439,455}$ and pheromone ${ }^{437}$ syntheses.

Good chemoselectivity was observed. A methylene exocylic ${ }^{468}$ or a terminal double bond, ${ }^{449}$ but also isolated $(E)$ olefins $^{443}$ and conjugated $(Z)$-enynes, ${ }^{120}$ are compatible with P2-Ni semihydrogenation of a triple bond.

Besides, it is interesting to recall that structure modifications can cause huge effects in terms of reactivity and selectivity. For instance, the synthesis of $13-\mathrm{F}_{4 \mathrm{t}}-\mathrm{NeuroP}$ by Taber et al. in 2008 showed that the semihydrogenation of a skipped triyne unit compared to its skipped yne-ene-yne analog behaves differently (Scheme 47). This example showed that, tuning the polyyne unit can overcome difficulties. ${ }^{441}$

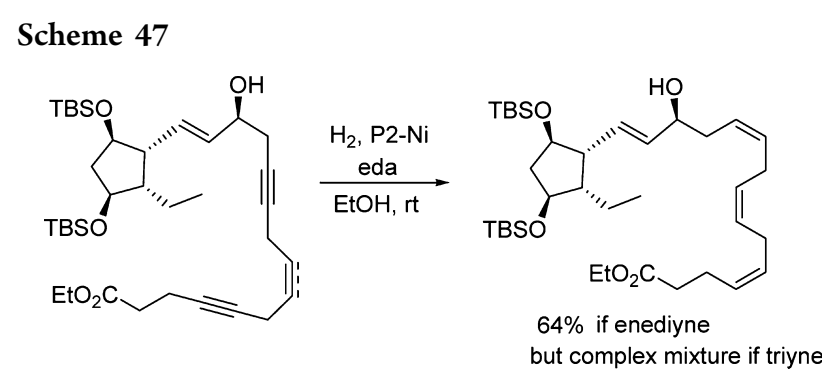

Partial reduction of a complex conjugated enyne is also a powerful way to access conjugated $(Z, Z)$ - or $(E, Z)$-natural products or key precursors. ${ }^{447,469}$ An interesting example is the synthesis of the C9-C29 fragment of ajudazol B by Ganame et al. This complex fragment could not be reduced using the Lindlar catalyst while a $55 \%$ yield was obtained with $\mathrm{P} 2-\mathrm{Ni}$ catalyst, along with a small amount of the C17-C18 fully saturated product (Scheme 48). ${ }^{120}$

Hulot et al. reported another remarkable partial reduction of a series of complex trienyne compounds leading in a one-pot operation to fenestradiene structures that could only be 
Scheme 48

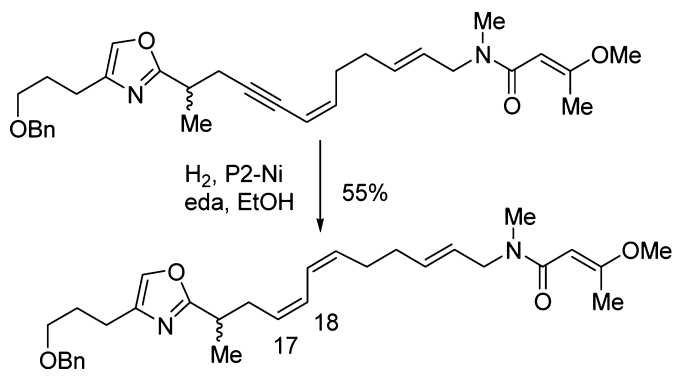

accomplished with P2-Ni (Scheme 49). ${ }^{470,471}$ The authors also showed that residual copper salts (copper being conveniently

\section{Scheme 49}

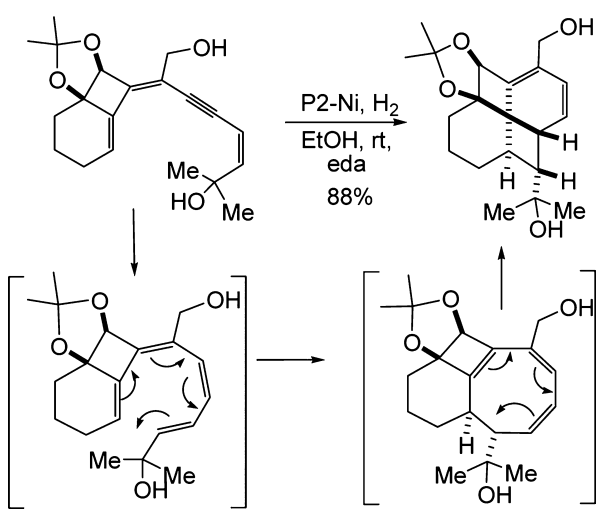

used to access the enyne structure via Sonogashira reaction) inhibited the reaction. Crude trienyne was therefore shaken vigorously with ethanolamine for $1 \mathrm{~h}$ to trap the copper salts through complexation.

As above-mentioned, chemoselectivity is very high with the Brown catalyst, but sometimes over-reduced products could not be avoided. It is therefore recommended to perform separation of compounds by chromatography on silver nitrate impregnated silica gel. ${ }^{443,458}$

3.3.1. The Case of TMS-Protected Alkynes. By contrast with the Lindlar catalyst, protection of a terminal alkyne with a TMS group does not systematically prevent the triple bond from a $\mathrm{P} 2-\mathrm{Ni}$ catalyzed reduction.

Indeed, in the presence of other internal alkyne functions, a nice regioselectivity was observed on a skipped triyne with a silylated-acetylenic end, affording the corresponding $(Z, Z)$ dienyne in $61 \%$ overall yield after subsequent desilylation with $\mathrm{AgNO}_{3}-\mathrm{KCN}$ (Scheme 50). ${ }^{472}$ On the contrary, Semeyn et al. showed that $(Z)$-vinylsilane derivatives were obtained in good yield from their corresponding TMS-alkynes. ${ }^{473}$ Besides, Chakraborty et al. observed that stereoselective partial

\section{Scheme 50}

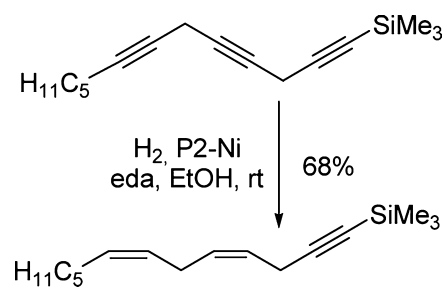

reduction of a TMS-alkyne function of a skipped diyne unit proved to be slower than the propargylic alcohol end. ${ }^{474}$ Nevertheless, when forcing the conditions, the desired diene was obtained in 53\% yield but, in that case, complete reduction of the propargylic alcohol was also observed to some extent.

Other examples were also reported, ${ }^{475}$ including a silylated derivative such as a $\mathrm{PhMe}_{2} \mathrm{Si}$-acetylenic compound which was semihydrogenated to its corresponding $(Z)$-vinylsilane in $74 \%$ yield $\left(24-48 \mathrm{~h}\right.$, on a large scale $(0.12 \mathrm{~mol}){ }^{476}$

3.3.2. Isomerization of (Z)-Double Bonds. Isomerization reactions are seldom reported. A few substrates were, however, prone to isomerization.

For instance, 3-aryl-2-propynols led to (Z)-3-aryl-2-propenols together with a nondescribed ratio of $(E)$-isomers. ${ }^{477}$ Similarly, stereoselective reduction of the triple bond of 4hexynylamine gave $(Z)$-4-hexenylamine with a small amount of the $(E)$-amine (13:1 ratio, respectively). ${ }^{478}$

3.3.3. Application to the Preparation of Labeled Polyenes. In 1981, Taber et al. prepared ${ }^{2} \mathrm{H}_{8}$-arachidonic acid via the partial hydrogenation of the corresponding skipped tetrayne precursor 5,8,11,14-eicosatetraynoic acid in 39\% yield. ${ }^{479}$ The authors used $\mathrm{NaBD}_{4}$ and EtOD as labeled reagent and solvent to achieve good deuterium incorporation $\left(58 \%\right.$ of $\left.d_{8}\right)$. Recently, Oger et al. showed that the enediyne unit could be deuterated without the used of such labeled reagents to address the synthesis of $d_{4}-4-\mathrm{F}_{4 \mathrm{t}}-\mathrm{NeuroP}$ with $98 \%$ of $d_{4}$ (Scheme 51$){ }^{431}$

\section{Scheme 51}
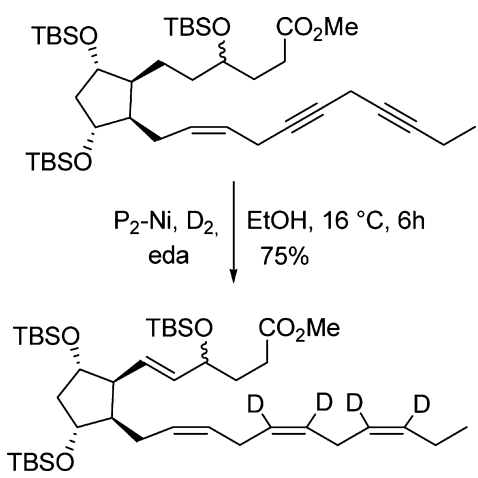

\subsection{Activated Zinc}

Of the many preparations described in the literature, ${ }^{218,480}$ the most often reported for the total synthesis of polyenes is the activation with 1,2-dibromoethane or activation as a zinccopper couple (or its variant zinc-copper-silver dust ${ }^{481}$ ). Some chemists prefer the use of the Rieke $\mathrm{Zn}^{249,250,482}$

The reduction using the $\mathrm{Zn}(\mathrm{Cu})$ couple is generally conducted in an aqueous methanolic mixture (1/ 1). ${ }^{237,331,481,483-485}$ Methanol may be replaced with dioxane ${ }^{485}$ or isopropanol. ${ }^{486}$ The presence of water (and its proportion) is, however, crucial. ${ }^{485}$ The reaction time ranges from a few hours $(5 \mathrm{~h})$ to overnight or $32 \mathrm{~h}^{281}$ or $48 \mathrm{~h}^{484}$ even, depending on the reaction temperatures (from $\mathrm{rt}^{281}$ to reflux ${ }^{237,485}$ ).

Various functional groups, such as methyl ester, ${ }^{330,482,487-489}$ triisopropyl ester, ${ }^{490}$ ether, $^{236}$ and ketones, ${ }^{236}$ OTBS, ${ }^{331,484}$ OTBDPS, 331,489 and OTBDMS, ${ }^{249}$ but also free hydroxyl groups, ${ }^{249,331,482,484}$ pyridine, ${ }^{484}$ and thiophene ${ }^{486}$ rings remain unaffected under the above conditions. The compounds containing several hydroxyl groups may be highly retained by 
the $\mathrm{Zn}(\mathrm{Cu})$ couple, thus lowering the yield of isolated compound. $^{239}$

However, as a limitation of this zinc methodology, attention should be paid to the fact that alkenes with powerful electronwithdrawing groups, such as esters, ketones, or nitriles, are reduced with a zinc-copper couple in refluxing methanol. ${ }^{491}$ Alkyl halides are also affected. ${ }^{492}$

Only a few examples were reported with a skipped diyne as the starting material (activation using 1,2-dibromoethane with or without copper bromide and lithium bromide). The reaction was incomplete in the case of a nonactivated diyne. ${ }^{493}$ The reduction was more efficient when one of the two alkynes is a propargyl function (nonprotected): the yields were usually high within short reaction times (purity GC and ${ }^{1} \mathrm{H}$ NMR generally $\geq 95 \%$ ). Side reactions were not observed. ${ }^{493}$ No example was found with neither skipped triynes nor tetraynes. Actually, the activated zinc system does not reduce nonactivated triple bonds. $^{237}$

Conversely, the partial reduction of 1,3-diyne is widely documented. Using an activated $\mathrm{Zn}(\mathrm{Cu})$ couple, the reaction is stereospecific and furnished the corresponding $(Z, Z)$-diene in good to excellent yields. ${ }^{331,484,487,494}$ This strategy was applied to the total synthesis of leukotriene $\mathrm{B} 4^{281}$ (a pro-inflammatory metabolite of arachidonic acid), (-)-(R)-haminol-1 (an alarm pheromone of a mediterranean mollusc) ${ }^{484}$ and amphidinol 3 (potent hemolytic properties, from marine dinoflagelates ${ }^{331}$ ).

The Rieke zinc reduction of conjugated enyols and diynols makes readily available a variety of $(E, Z)$ - and $(Z, Z)$-1,3-dienes in excellent yields, without any problem of over-reduction. ${ }^{249}$ This methodology (reflux in THF/ $\mathrm{MeOH} / \mathrm{H}_{2} \mathrm{O}: 7 / 5 / 1,2-4$ equiv, $30 \mathrm{~min}$ to $40 \mathrm{~h}$ ) proved to be highly cis-selective and provided convenient access to 3-hydroxyleukotriene $\mathrm{B} 4{ }^{482}$ Besides, isolated alkynes are much less reactive than alkyne activated by conjugation with a double bond, another triple bond, or an $\alpha$-alkoxy function, thus leading to a high chemoselective reaction when both types of alkynes are present in the same molecules (Scheme 52). ${ }^{249}$

\section{Scheme $52^{249}$}

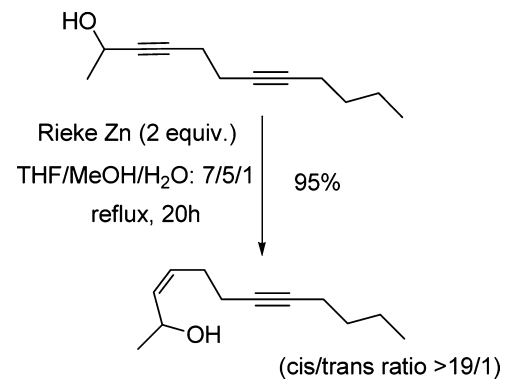

Activation with 1,2-dibromoethane in refluxing ethanol (referred as to $\mathrm{Zn}^{*}$ ) appeared to be not sufficiently reactive with 1,3-diyne: the reaction is often incomplete, leading to an enyne instead of the expected $(Z, Z)$-dienes. In some cases, this poor reactivity is turned to an advantage, allowing high regioselectivity. Effectively, steric hindrance (tert-butyl group at the $\alpha$-position or a TMS-alkyne protection) prevented the reduction while the presence of an alkoxy-function at an $\alpha$ position favored it, in detriment to the second triple bond. ${ }^{493}$

Unfortunatly, in some runs, besides the lack of reactivity of the nonactivated alkyne groups, the reaction was not stereoselective: Scheme 53 shows that, besides the high regioselectivity, the expected ( $Z$ )-allylic alcohol is obtained (80\%) together with $20 \%$ of its $(E)$-isomer. ${ }^{493}$

\section{Scheme $53^{493}$}

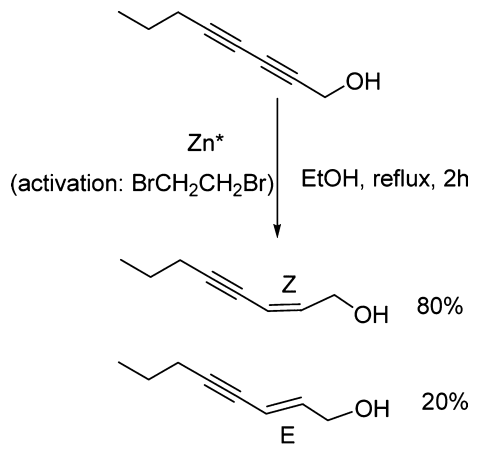

As illustrated in Scheme 54, the activated zinc methodology was successfully applied to conjugated 1,3,5-triynes, providing the desired $(Z, Z, Z)$-trienes in good yield. ${ }^{495}$

\section{Scheme $54^{495}$}

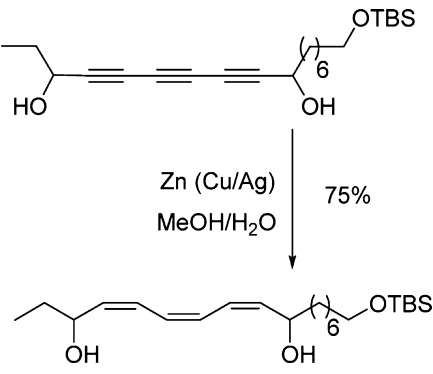

Besides an usually high stereoselectivity, ${ }^{486}$ the conditions of the reaction also led to a high chemoselectivity in the presence of other double bonds. (E)-Enynes are selectively converted to their corresponding $(E, Z)$-dienes. ${ }^{481}$ Interestingly, the reaction was very clean, and further purification was not necessary. ${ }^{485}$ It is noteworthy that $\alpha$-branched enynes are reduced with difficulties only. ${ }^{237}$ The selective reduction of enyne was a key step in the synthesis of dienol sex pheromones ${ }^{45}$ and 11cis-retinoids (Scheme 55). ${ }^{242}$ Likewise, an excellent chemoselectivity is observed in the presence of an isolated olefin $^{121,439,488}$ or terminal diene, ${ }^{486}$ a $(E, E)$-diene, ${ }^{433,488,490}$ a $(Z, E)$-diene, ${ }^{237,472}$ a $(E, E, E)$-triene, ${ }^{237}$ or a $(E, E, Z)$-triene. ${ }^{237}$

These selective reaction conditions were widely used in the synthesis of di- or trihydroxylated metabolites of PUFAs such as leukotriene $\mathrm{B} 4^{240,488,496}$ (Scheme 56 ) or even a ${ }^{13} \mathrm{C}$-labeled

\section{Scheme 55}

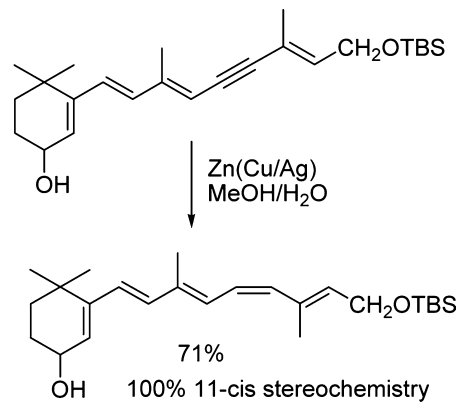


analog $^{489}$ diHETE, ${ }^{121}$ HETE, $^{439}$ or resolvin E $1^{490}$ (Scheme 57) but also recently resolvins E3, ${ }^{497} \mathrm{E} 2,{ }^{498} \mathrm{D} 6,{ }^{499}$ and neuroprotectin D1. ${ }^{500}$

\section{Scheme $56^{240}$}

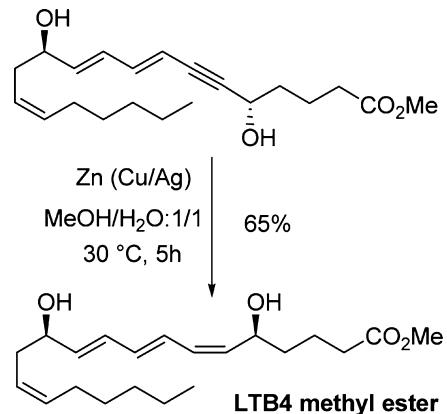

Scheme $57^{490}$
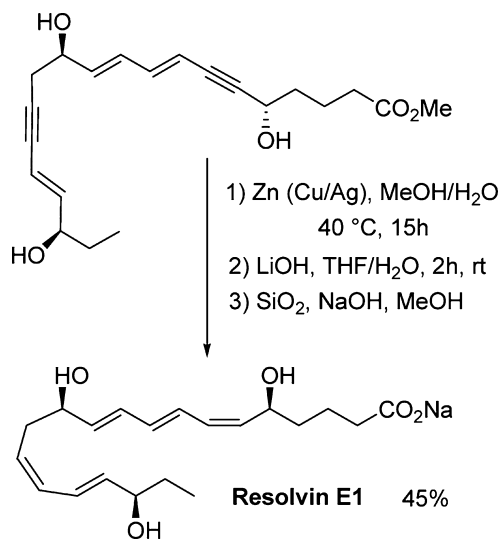

Recently, Mohamed et al. described ${ }^{330}$ the total synthesis of methyl bosseopentaenoate, a natural eicosapentanoate from red and green alga (Lithothamnion corallioides and Anadyomene stellata) (Scheme 58) and also pointed out the good selectivity

Scheme $58^{330}$

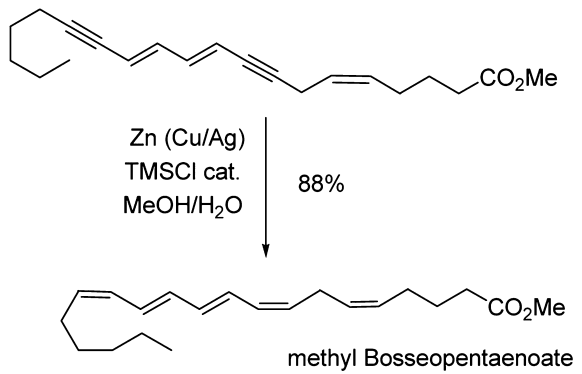

(isolated from red and green marine alga)

of these zinc-copper couple reaction conditions. Addition of a catalytic amount of TMSCl in aqueous methanol reduced the reaction time and improved the stereoselectivity. ${ }^{330}$

Interestingly, since the presence of free hydroxyl groups is well-tolerated, the reduction reaction is often conducted in a late stage of the strategy. This is a crucial criterion for enabling incorporation of radioactive atoms.

In 1994, Boland et al. suggested selective semideuteration reactions of conjugated alkynes using deuterated solvents and $\mathrm{Zn}(\mathrm{Cu} / \mathrm{Ag}){ }^{243}$ Unfortunately, the structures of the alkyne precursors were not clearly mentioned. Neither yields nor deuterium incorporation percentage were given. In addition, it seems that a complete deuteration was nevertheless difficult and some hydrogen/deuterium atom exchange was observed for the deuteration of terminal alkynes.

A few years later, Spur and co-workers successfully applied the $\mathrm{Zn}(\mathrm{Cu} / \mathrm{Ag})$ method in $\mathrm{CD}_{3} \mathrm{OD} / \mathrm{D}_{2} \mathrm{O}$ to the selective partial deuteration of an en-yne-diene structure, yielding the 11,12-dideuterio-15-epi-LXA4 methyl ester. ${ }^{246}$ The deuteration proceeds cleanly, provided the $\mathrm{Zn}(\mathrm{Cu} / \mathrm{Ag})$ alloy was previously activated by $2 \mathrm{~N} \mathrm{HCl}$.

Similarly, the Petasis team also prepared many deuterated metabolites of PUFAs, such as resolvin $\mathrm{E}^{501,502}$ and LXA4, ${ }^{501}$ for example. No deuterium scrambling was observed in the ${ }^{1} \mathrm{H}$ NMR spectrum of the 6,7,14,15-deuterated resolvin E1. ${ }^{501}$

Application to tritium labeling was performed by Petasis and co-workers using tritiated water. ${ }^{501,502}$ It is, however, not clearly specified whether the solvent must also be tritiated or whether the use of a nonlabeled solvent is sufficient to obtain enough radioactivity.

Unfortunately, no information regarding the percentage of labeled atom incorporation and the efficiency of the reaction (conversion, yield, byproducts) is given in the above-mentioned deuteration and tritiation reactions.

\subsection{Hydroboration Reaction}

This method, also called the Brown-Zweifel procedure, ${ }^{160}$ has been applied to the synthesis of labeled PUFAs for many years. ${ }^{159}$ However, only a few teams employed this methodology to the synthesis of polyenes structures.

The hydroboration reaction is usually carried out in anhydrous $\mathrm{THF}^{503-506}$ or diglyme. ${ }^{507-510}$ The alkenyl-boron intermediate is then quenched under strong basic conditions and then oxidized with $\mathrm{H}_{2} \mathrm{O}_{2} 30 \%$. Prior to protonolysis, some authors also quenched the excess of borane reagent with ethylene glycol. ${ }^{159,509,510}$

The reaction seems highly stereoselective, tolerating methyl esters, ${ }^{507-509,511}$ benzoyl esters, ${ }^{512}$ OTHP,${ }^{481,505,513}$ OTBS, ${ }^{512}$ acetal, $^{514}$ bromine atoms, ${ }^{503}$ acetonides, ${ }^{504}$ and deuterium ${ }^{508}$ atoms. Some examples are also reported with a free hydroxyl group $^{515,516}$ (which required a large excess of the borane reagent). Besides, it is important to recall that, under the standard conditions (THF, $0{ }^{\circ} \mathrm{C}$ ), dialkylboranes react with many functional groups: aldehyde, ketones, $\gamma$-butyrolactone, and isocyanate are reduced rapidly. ${ }^{517}$ Carboxylic acid and primary amide evolve gas without undergoing reduction whereas tertiary amides ${ }^{518}$ are cleanly converted to aldehydes. Nitrile and DMSO are somewhat more resistant. ${ }^{517}$ Esters are inert.

Several boranes were employed, mainly the catecholborane ${ }^{511}$ dicyclohexylborane-dimethylsulfide complex, ${ }^{503-506,515}$ disiamylborane $\left(\mathrm{Sia}_{2} \mathrm{BH},\right)^{159,507,509,510,512,519}$ but also the dibromoborane-dimethylsulfide complex. ${ }^{411}$ They are all prepared in situ at low temperatures by dropwise addition of the corresponding alkene to a solution of borane-sulfide complex in THF, or a mixture of boron trifluoride-diethyl ether and sodium borohydride.

Interestingly, excess of borane reagent may be added to ensure a complete reduction of the alkyne. ${ }^{511}$

In addition, in the papers that are reviewed herein it has not been mentioned what motivated the choice of one borane rather than another one. Thus, it is important to recall that boranes readily add to alkenes, with the rate of the reactivity 
being dramatically influenced by the steric or electronic effects and the type of borane employed. For instance, 9-BBN is sensitive to electronic effects that diisoamylborane ignores. Reversely, the reaction of $\mathrm{Sia}_{2} \mathrm{BH}$ is controlled primarily by steric effects whereas 9-BBN reduced hindered double bonds efficiently (THF, rt). ${ }^{520}$

In addition, the cis-alkenes undergo hydroboration with $\mathrm{Sia}_{2} \mathrm{BH}$ at a rate considerably faster than the one of the corresponding trans-alkenes. It is therefore fascinating to discover that the reverse is true for $9-\mathrm{BBN}^{520}$

Thus, the choice of the borane may be crucial for the selective reduction of alkynes in the presence of other olefins (or polyenes).

The Brown-Zweifel procedure was successfully employed for the partial reduction of skipped diynes ${ }^{159,507,511-513,521}$ and skipped triynes. ${ }^{159,503,504,508,512,515}$ Scheme 59 is given as an example. Sometimes the yields are, however, moderate $\left(54 \%{ }^{509} 56 \%{ }^{515} 65 \%{ }^{503}\right.$ or not reported $\left.{ }^{508,512}\right)$.

Scheme $59^{159}$

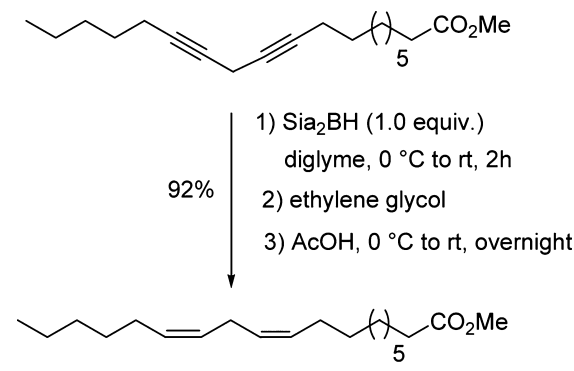

Interestingly, although double bonds react with boranes (for instance, $\mathrm{Sia}_{2} \mathrm{BH}^{218}$ ), a good chemoselectivity toward alkyne functions could be reached in the presence of alkene(s).

For instance, using $\mathrm{Sia}_{2} \mathrm{BH}$ in diglyme, Sgoutas et al. reported $^{159}$ the reduction of $(Z)$ - or $(E)$-enynes with excellent yields (81-94\%). Mori et al. also obtained good results with a $(Z)$-enyne. ${ }^{514}$ The reaction is also chemoselective in the presence of a silylated $(E)$-allylic alcohol. ${ }^{512}$

Thus, the hydroboration-hydrolysis procedure was applied to the synthesis of metabolites of PUFAs such as the $\left[14,14-{ }^{2} \mathrm{H}_{2}\right]$ linolenic acid ${ }^{509}$ (deuterated at a methylene bisallylic position) and the $5(\mathrm{~S})-\mathrm{HETE}^{512,521}$ (Scheme 60) or $12(\mathrm{~S})$-HETE. ${ }^{521}$ No skipped tetrayne reduction via hydroboration reaction was found in the literature.

Scheme $60^{521}$

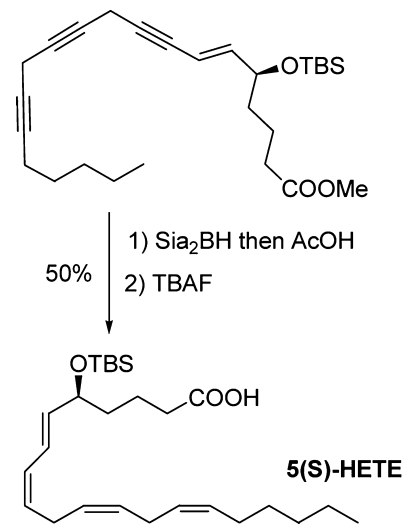

Actually, it has also been reported that the hydroboration rate for alkenes and alkynes is similar when catecholborane is used as hydroborating reagent under uncatalyzed thermal conditions. Consequently, low chemoselectivity is observed. ${ }^{180}$ Although a plethora of different boranes and catalysts for hydroboration reactions can be found in the literature, few examples circumvent the lack of selectivity for alkynes. ${ }^{180}$ Pelter et al. reported ${ }^{522}$ dimesitylborane as a selective hydroboration agent for alkynes, but this reagent has not been used in the chemoselective total synthesis of polyenes.

Hydroboration of 1,3-diynes furnished their corresponding conjugated $(Z, Z)$-dienes as useful precursors in the synthesis of pheromones. ${ }^{481,506,516}$ The effectiveness of this procedure is illustrated in Scheme 61.

\section{Scheme 61}
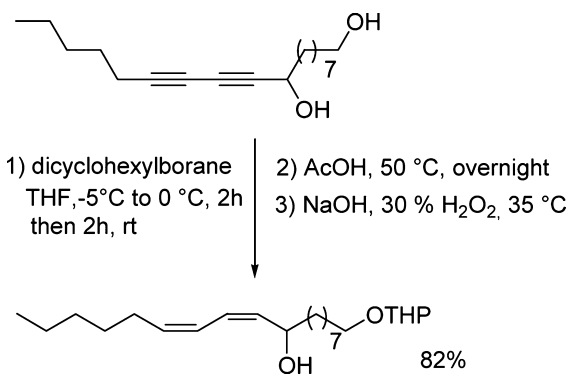

However, this reduction does not seem to be always stereospecific, since, in some cases, traces of the conjugated $(Z, E)$-diene contaminated the sample after a first column chromatography on silica gel (mixture with $2 \%$ of $(Z, E)$-diene, $30 \%$ of $(Z, Z)$-diene and $68 \%$ of cyclohexanol). Obtention of the expected pure $(Z, Z)$-diene (37\% yield) required a further purification step on $\mathrm{AgNO}_{3}$-impregnated silica gel. ${ }^{506}$

It is noteworthy that the addition of 1 equiv of $\mathrm{Sia}_{2} \mathrm{BH}$ to a symmetrical 1,3-diyne led to enynes in good yield (74$76 \%) .{ }^{519}$ However, the addition of a 2 nd equiv to the monohydroboration product derived from dodeca-5,7-diyne was very slow. This difficulty could be circumvented by using the sterically less-hindered dicyclohexylborane. ${ }^{519}$

Contrary to what is observed with most of the methods for obtaining ( $Z$ )-alkenes from alkyne, over-reduction does not occur, since the resulting alkenylborane intermediate is electron poor and sterically hindered. ${ }^{511}$ No trans-isomer was observed, and no under-reduced alkyne remains, since excess of hydroboration reagent may be added if necessary. ${ }^{511}$ These aspects of the Brown-Zweifel procedure are rather interesting, since separation of compounds that differ only in one element of unsaturation would require an HPLC separation step.

3.5.1. Application to the Preparation of Labeled Polyenes. Deuteroboration of skipped diynes or skipped triynes using bis(2-deuteriocyclohexyl)borane-B- $D_{1}$ followed by deuterolysis with $\mathrm{CH}_{3} \mathrm{CO}_{2} \mathrm{D}$ provided deuterium labeled polyunsaturated fatty acids: for instance, synthesis of methyl linoleate $(Z, Z)$-diene, $53 \%$ yield, $98 \% \mathrm{D}$ atom $)^{510}$ or preparation of a skipped $(Z, Z, Z)$-triene tetrahydropyranyl ether (66\% after THP deprotection, Scheme 62$)$ as the precursor of linolenic acid. ${ }^{505}$

Alternatively, Svatos et al. also prepared derivatives bearing only one deuterium atom on each double bond by using nondeuterated dicyclohexylborane and then $\mathrm{CH}_{3} \mathrm{CO}_{2} \mathrm{D}$. ${ }^{505}$ 
Scheme 62

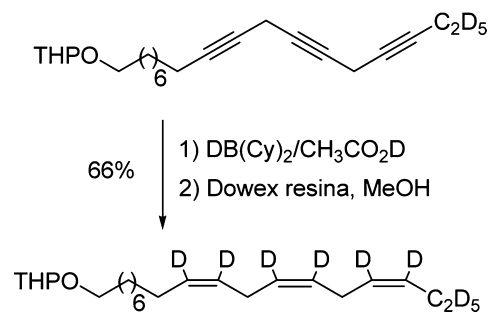

In order to achieve approximatively $4 \%$ labeling of a diyne, the deuterated acetic acid had to be present at $20 \%$ in the acetolysis mixture $\left(\mathrm{CH}_{3} \mathrm{CO}_{2} \mathrm{D} / \mathrm{CH}_{3} \mathrm{CO}_{2} \mathrm{H}\right) .{ }^{511}$

No deuterium scrambling was observed. That is a notable advantage of this deuteroboration over the other deuteration reactions. Effectively, deuterium scrambling is particularly troublesome in isotopic effects measured using scintillation counting and mass spectroscopy. ${ }^{505,511}$

Svatos et al. ${ }^{505}$ emphasized that the final products were virtually free $(<0.5 \%$ by GC) of trans-isomers and of over- or under-reduced material. Consequently, unlike other hydrogenation or reduction methodologies, no further purification steps were necessary. ${ }^{505}$

Tritiation of a skipped diyne was accomplished ${ }^{507}$ using 2methylbut-2-ene in diglyme and sodium borotritide $(150 \mu \mathrm{Ci}$, diluted with sodium borohydride). A tritiolysis (tritiated acetic acid, $200 \mu \mathrm{Ci}$, overnight) followed by a protonolysis (acetic acid, $8 \mathrm{~h}$ ) afforded the desired 1,4 -diene $\left({ }^{3} \mathrm{H}\right.$ specific activity $1026 \mu \mathrm{Ci} \mathrm{mmol}^{-1}$ ) although the yield was rather poor (34\% yield after HPLC).

Yields of the tritioboration reaction using tritiated borane derivatives were somewhat lower than those recorded with unlabeled reagents, and the yield seems to decrease with increasing specific activity of the tritiated sodium borohydride employed. $^{159}$

\subsection{Titanium-Mediated Reductions}

Reduction employing titanium/magnesium-based reagents is a much less popular method for preparing $(Z)$-alkenes than the hydrogenation via heterogeneous catalyst $(\mathrm{Ni}, \mathrm{Pd})$.

However, this titanium-mediated method for the $(Z)$ reduction of alkynes was applied by Kitching ${ }^{253,254}$ to a variety of conjugated or skipped diynes and skipped triynes.

The reaction is performed at low temperature $\left(-70{ }^{\circ} \mathrm{C}\right.$ then warmed up to $-30{ }^{\circ} \mathrm{C}$ ) and required anhydrous conditions $\left(\mathrm{Et}_{2} \mathrm{O}\right.$, substrate). It is more convenient (and less dangerous) than the use of hydrogen gas.

Attention must be paid to the functional groups of the starting substrate. Indeed, esters are affected by the reaction conditions. Kulinkovich ${ }^{523}$ showed that methyl ester are converted to 1-substituted cyclopropanol in the presence of EtMgBr (2 equiv) and a catalytic amount $(5-10 \% \mathrm{~mol})$ of titanium isopropoxide $(76-95 \%$ yields). Thus, the synthetic route to natural products containing an ester or acid function should envision its introduction after this titanium basedreduction reaction.

Similarly, carbonyl functions should be avoided. The alkoxytitanacyclopropene intermediate is reactive enough toward aldehydes and ketones to yield allyl alcohols. ${ }^{252}$ Moreover, due to the addition of alkylMgBr, the presence of free alcohol, amines, and thiols must be excluded. Protective groups such as $\mathrm{EE}^{252}$ and $\mathrm{OTHP}^{253,254}$ were suggested.
The reaction proved to be highly stereoselective, providing $(Z)$-olefins only. In addition, the reduction process does halt after complete consumption of the alkyne, even when a large excess of Ti-and Mg-reagents is added (Scheme 63). Knowing

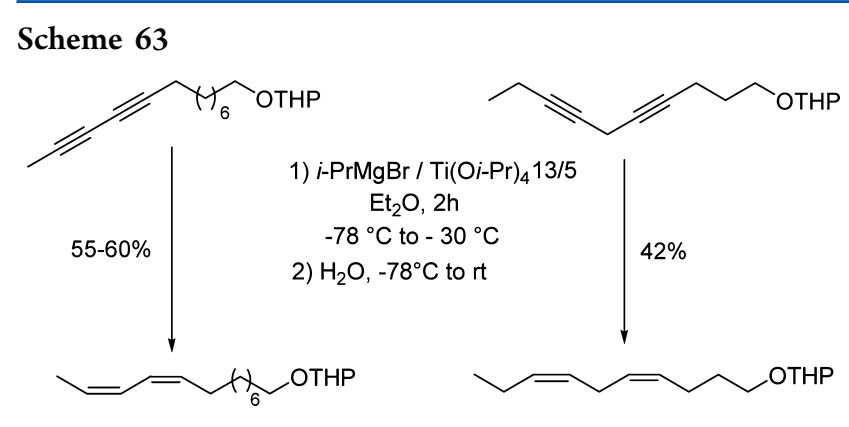

the difficulties to purify mixtures containing over- and underreduced byproducts, this absence of over-reduction makes a big difference with the other methodologies based on palladium or nickel catalysts.

On the whole, the yields are moderate only, even if sometimes rather low (25 to $42 \%$ ), despite an extremely large excess of both the $\mathrm{Ti}-$ and $\mathrm{Mg}$-reagents (the $\mathrm{Ti}(\mathrm{O} i \mathrm{Pr})_{4} / i$ $\mathrm{PrMgBr}$ ratio ranges from $11 / 4$ to $20 / 8$ per mole of starting alkynyl compound). Indeed, excess reagents were utilized to ensure completion of the reaction. ${ }^{254}$ Thus, obviously, many side-reactions occurred.

The reaction is also highly chemoselective with respect to terminal or $(E)$-double bonds. An example is depicted in Scheme 64.

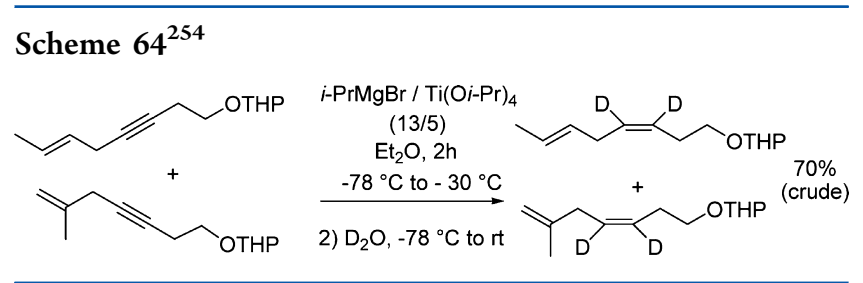

Starting from a TMS-diyne, the reduction showed no regioselectivity. Thus, both triple bonds were semireduced (Scheme 65), unlike the hydrogenation over the Lindlar catalyst, which was sensitive to the bulkiness of the silyl group (see section 3.1).

The titanium-mediated reduction of alkyne was a key step in the total synthesis of several natural products, such as linolenic acid, oleic acid, and (3E,8Z,11Z)-tetradeca-3-8-11-trienyl acetate, the sex pheromone of a tomato pest scrobipalpuloides absoluta.

\section{Scheme 65}

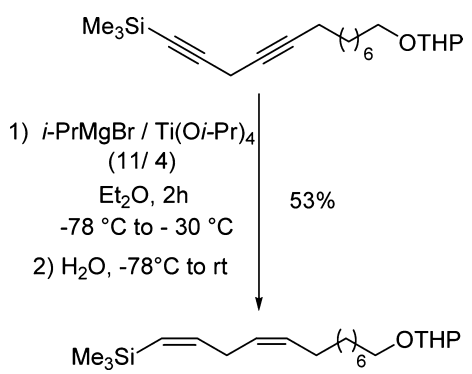


Replacement of water with deuterium oxide when quenching the reaction provided the corresponding deuterated analogs, although in much lower yields (Scheme 66). Interestingly, no scrambling of deuterium was detected by NMR or GC-MS analysis. $^{254}$

Scheme 66

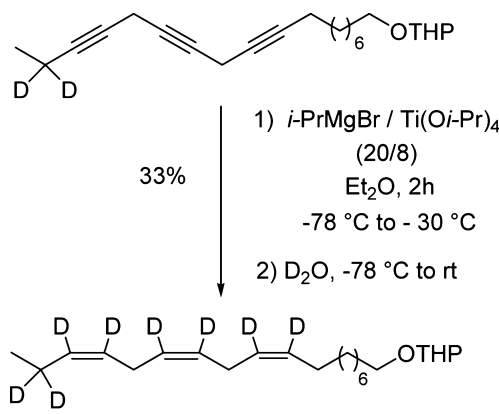

Actually, besides the moderate to poor yields, this process also suffers from a serious drawback, since a skipped diyne system turned into a 1,3-diene during the course of the reduction of an isolated alkyne. Unfortunately, the expected triene (Scheme 67) was contaminated with a significant amount of two nonseparable isomers (percentage not given). ${ }^{254}$

Scheme $67^{254}$

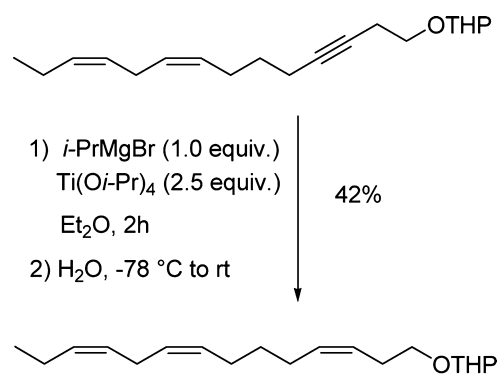

contaminated with 2 minor isomers lacking the 'methylene-skipped' diene system

Interestingly, in another approach using 2.0 equiv (instead of a catalytic amount) of titane(IV) alkoxide and $n$-BuLi (instead of organomagnesium bromide), Lara-Ochoa et al. ${ }^{524}$ reported the synthesis of anticancer drugs Combretastatins A4 and AVE$8062 \mathrm{~A}$ without protecting the aniline or phenolic functions. Minor amounts of the corresponding alkane were nevertheless observed. The methods also tolerated OMOM protection.

Actually, the addition of $n$ - BuLi is performed at $-78{ }^{\circ} \mathrm{C}$, but the temperature is then raised to $+50{ }^{\circ} \mathrm{C}$. This methodology is thus restricted to molecules which can resist such drastic conditions. In this reaction, $n$-BuLi acts as a reducing agent for the conversion of $\mathrm{Ti}(\mathrm{IV})$ to $\mathrm{Ti}(\mathrm{II})$ and its replacement with other titanium alkoxides or organomagnesium bromides failed (in that case, the intermediate titanium(II)-alkyne complex is not thermally stable). ${ }^{525}$

Examples of neither diyne reduction nor regio- nor chemoselectivity were found in the literature.

\subsection{Other Methods}

3.7.1. Hydroalumination. Hydroalumination reaction is mainly reported for obtaining alkenylalanes ${ }^{208,526,527}$ as useful precursors for subsequent metal-mediated cross-coupling reaction. In some cases, however, upon hydroalumination of internal alkynes with DIBAL-H, subsequent protiolytic workup affords $(Z)$-alkenes. Upon addition of the reagents at $0{ }^{\circ} \mathrm{C}$, the reaction takes place at $\mathrm{rt}$ in anhydrous heptane for $12-15 \mathrm{~h}$.

This method was applied in 1963 to the synthesis of positional isomers of linoleic acid. ${ }^{528}$ Neat DIBAL-H reagent reacted smoothly with skipped diynes to furnish the desired chloro-(1Z,4Z)-dienes in excellent yields (82-94\%). It is noteworthy that neither hydrogenation over $\mathrm{Pd} / \mathrm{CaCO}_{3}$ catalyst nor reduction with a zinc-copper alloy in alcohol proved suitable in that case.

In addition, if the substrate has both an alkyne and an alkene group, the hydroalumination step showed chemoselectivity in favor of the alkyne. ${ }^{526,529}$

However, despite the appealing above-mentioned yields and stereoselectivity and chemoselectivity toward alkenes, this process was applied to a relatively small extent in multistep syntheses.

Several drawbacks may explain why chemists are reluctant to use DIBAL-H as a reductive agent for obtaining $(Z)$-alkenes:

(i) DIBAL-H reacts with many functional groups, ${ }^{218}$ especially amide, nitriles, tosylates, epoxides, and esters, which are often encountered in natural products or bioactive molecules.

(ii) Any protic functions must be protected or a big excess of DIBAL-H must be added. ${ }^{530,531}$

(iii) Alkenylalanes tend to dimerize to afford a butadiene unit. $^{532}$

3.7.2. Tri(n-butyl)boron. (4Z,7Z)-Undecadiene was prepared from its corresponding skipped diyne and $n$-tributyl boron. ${ }^{533}$ The temperature is raised quickly from ca. 100 to 160 ${ }^{\circ} \mathrm{C}$. Gas evolution (butane) is evolved. After distillation of unreacted tri(n-butyl)boron and diyne, protonolysis is performed with acetic acid.

The extremely high temperatures required in this procedure have certainly dampened any motivation to apply it to the reduction of sensitive polyfunctionalized alkynes.

3.7.3. $\mathrm{H}$-Transfer Using $(\mathrm{dba})_{3} \mathrm{Pd}_{2} \cdot \mathrm{CHCl}_{3}$ Catalyst. A large number of homogeneous transition metal complexes have been reported as catalysts for the stereoselective hydrogenation of alkynes. ${ }^{24}$ However, this reducing method is seldom applied in multistep syntheses.

Trost et al. showed ${ }^{283}$ that the $\operatorname{Pd}(0)$-acetic acid catalytic system provides a mild and stereospecific method for alkyne reduction to pure $(Z)$-isomer olefin.

Application to a skipped diyne afforded its corresponding $(Z, Z)$-diene in $59 \%$ yield together with a nonidentified byproduct (Scheme 68).

Only one example of diyne reduction is given. It is thus difficult to estimate the effectiveness of this reduction process as a key step in the total synthesis of highly functionalized polyenes.

Furthermore, it is important to highlight that, starting from ynoates, 5-decyne, or TMS-protected alkynes, Trost et al. obtained $^{283}$ a non-negligible amount of the $(E)$-isomers $(Z / E$ ratios ranging from $6.8 / 1$ to $1 / 3.7$ )

Thus, besides the necessity of running the reaction in benzene, the possibility of $(Z / E)$-isomerization has probably dissuaded most chemists from using this Pd-mediated homogeneous reduction system in multistep syntheses of sensitive bioactive compounds. 


\section{Scheme 68}

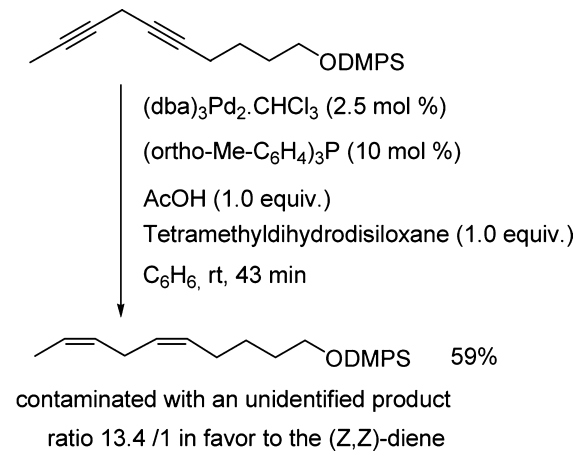

3.7.4. Diimide. The use of diimide as reducting agent for obtaining a $(Z)$-alkene from an alkyne is not often reported in the recent literature. In total synthesis, diimide is mainly employed for the conversion of 1-haloalkyne to (Z)-vinylhalides in good yields. ${ }^{534-537}$

In 2005, in the course toward the synthesis of fostriecin, a 2,4,6-tri-isopropylbenzenesulphonyl hydrazide as diimide precursor under mild basic conditions afforded a cis-vinylsilane from a silylated-protected alkyne whereas previous other attempts to effect this transformation were hampered by either low yield and/or over-reduction. ${ }^{538}$ A free hydroxyl group and O-silylated protecting groups were tolerated. A good stereoand chemoselectivity were observed in the presence of a $(E)$ double bond (Scheme 69), although the yield is moderate only (incomplete conversion).

\section{Scheme 69}

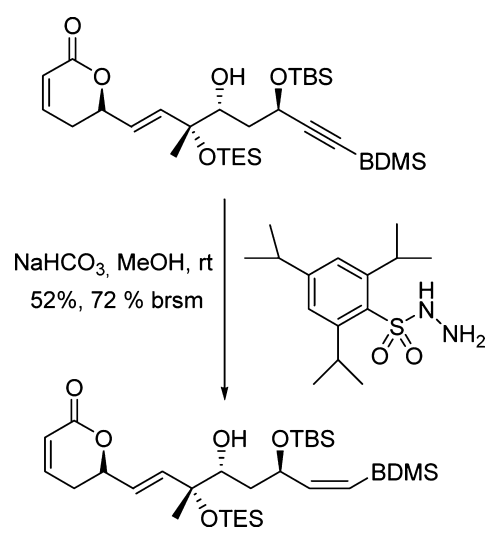

\section{COMPARISON OF REDUCING METHODS}

Problems of reactivity (over-reduction, under-reduction) or poor chemoselectivities led some authors to perform several methods prior to success in obtaining the desired pure target.

Thus, in this section, we review comparative studies, with the hope that it can help chemists in choosing the most suitable reductive process for their novel molecules.

\subsection{Preference for the Lindlar Catalyst}

Huang et al. ${ }^{539}$ highlighted the difficulty in finding the exact conditions to selectively reduce a skipped triyne to its corresponding $(Z, Z, Z)$-triene. While the authors successfully and routinely performed semihydrogenation of skipped diyne to diene using $\mathrm{P} 2-\mathrm{Ni}$ catalyst, extrapolation of their reaction conditions to a skipped triyne analog was disappointing, leading to a complex mixture containing at least four components in equal amounts.

The "modified" Lindlar catalysts such as $\mathrm{Pd} / \mathrm{BaSO}_{4}$ or Lindlar catalyst with triethylamine also gave poor results, comparable to those obtained under their $\mathrm{P} 2-\mathrm{Ni}$ conditions (details not reported).

For them, it turned out that the best results were obtained with the "classical" Lindlar catalyst (EtOH, two drops of quinoline in a $0.333 \mathrm{mmol}$ scale, temperature not mentioned). Thus, the desired skipped triene was isolated in $83 \%$ yield (after preparative TLC).

Lindlar catalyst is the most frequently used procedure, and thus, it seems to imply that it is the most suitable and efficient process for obtaining $(Z)$-alkene from alkyne.

However, according to the above-mentioned Huang et al. comments, ${ }^{539}$ Spinella et al. ${ }^{368}$ reproduced the same procedure to partially reduce a skipped triyne in a structure which also contains a TBSO-protected (Z)-allylic alcohol (Scheme 70).

Scheme $70^{368}$
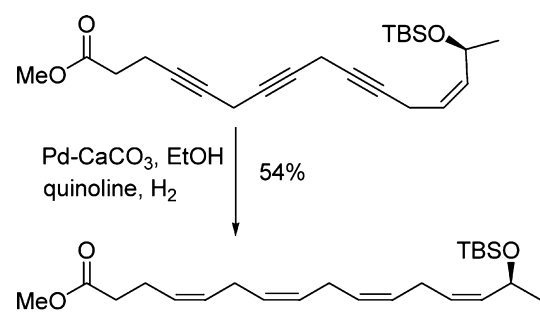

The desired target was effectively obtained, but in moderate yield only ( $54 \%$ yield), showing evidence that the chemoselectivity of the hydrogenation reaction is never guaranteed and may vary markedly with even small changes in the structure of the substrate.

Actually, while the hydrogenation reaction over Lindlar catalyst is the most famous and employed reaction for obtaining $(Z)$-alkenes from alkynes, very few chemists reported that they preferred this procedure in comparison with other methods. Thus, it is difficult to know whether most of the chemists who performed such a reduction do prefer this reaction process or whether they managed with it somehow and, thus, they probably did not try comparison with other reducing systems.

Indeed, the next paragraphs highlight that many chemists are disappointed with the results carried out under the Lindlar conditions.

\subsection{Preference for the Rosenmund Catalyst}

Saniere et al. ${ }^{414}$ found that the selective hydrogenation was more efficient (60\% yield) with the $\mathrm{Pd} / \mathrm{BaSO}_{4}$ system in the presence of quinoline than the Lindlar catalyst, which converted a starting "double" diyne into the corresponding bis-diene in very poor yield.

Tallman ${ }^{540}$ et al. considered the Lindlar catalyst as a mild catalyst in comparison with the $\mathrm{Pd} / \mathrm{BaSO}_{4}$ system, which is considered as a "more reactive catalyst". They employed one or the other depending on the starting substrate. Besides, in the presence of a TMS-protected ( $Z$ )-enyne, the semihydrogenation reaction of an isolated internal alkyne proceeded slowly using a $\mathrm{Pd} / \mathrm{BaSO}_{4}$ system desactivated with quinoline or the Lindlar catalyst whereas it was highly efficient ( $94 \%$ yield) but also chemo- and regioselective (TMS-protected $(Z)$-enyne not affected) using $\mathrm{Pd} / \mathrm{BaSO}_{4}$ in $\mathrm{EtOAc}^{423}$ 
Starting with a skipped triyne, Hnarnachari ${ }^{541}$ first carried out $\mathrm{P} 2-\mathrm{Ni}$ reaction conditions. The hydrogenation conditions were apparently not efficient enough, providing an underreduced intermediate, which was then reduced to the expected skipped $(Z, Z, Z)$-triene using the $\mathrm{Pd} / \mathrm{BaSO}_{4}$ hydrogenation process. The authors did not comment on this reaction sequence (Scheme 71), but it seems to imply that the NHBoc

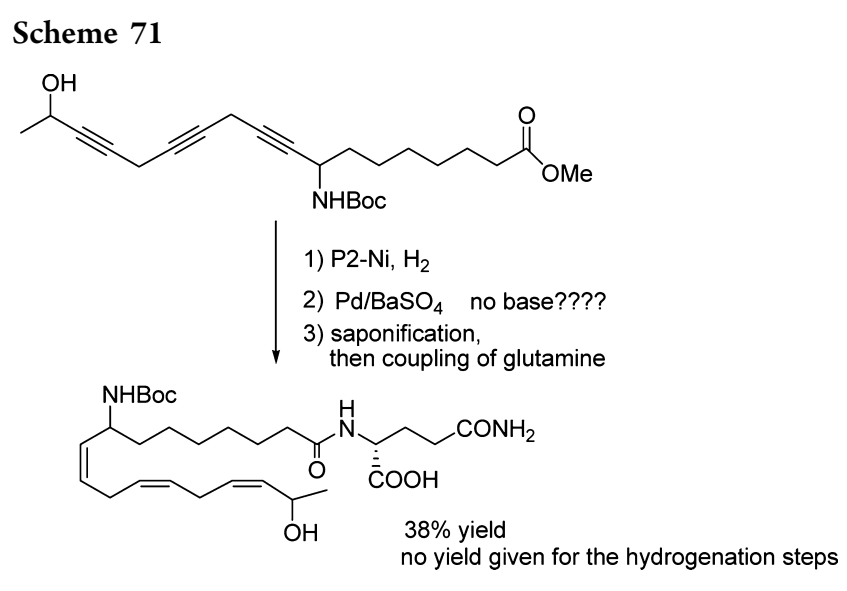

function disturbs the course of the reaction. Actually, in a previous paper, ${ }^{436}$ starting from a similar triyne which did not contain the NHBoc group, they reported a successful hydrogenation reaction using the same $\mathrm{P} 2-\mathrm{Ni}$ conditions.

\subsection{Preference for the Brown Catalyst}

Several authors concluded that partial hydrogenation with ethylenediamine poisoned $\mathrm{P} 2-\mathrm{Ni}$ catalyst was far superior than other reduction methods. ${ }^{116,308,416}$ For instance, starting from a diyne containing both $\mathrm{CH}_{2} \mathrm{OH}$ and $\mathrm{CH}_{2} \mathrm{OTBS}$ groups, Feutrill et al. $^{308}$ preferred the $\mathrm{P} 2-\mathrm{Ni}$ catalyst rather than the Lindlar catalyst, which gave erratic results.

In addition, using a $\mathrm{P} 2-\mathrm{Ni}$ procedure to reduce a skipped diyne, Dussault et al. ${ }^{416}$ obtained the expected diene (68\% yield), although it was contaminated with $5 \%$ of a nonidentified isomeric impurity. Attempts with $\mathrm{Pd} / \mathrm{CaCO}_{3}$ or $\mathrm{Pd} / \mathrm{BaSO}_{4}$ occur with significantly poorer selectivity.

Moreover, the Brown catalyst appeared to be more reactive and less sensitive to steric hindrance than the Rosenmund catalyst. Thus, starting from a skipped diyne substituted with a tert-butyl group, ${ }^{542} \mathrm{P} 2-\mathrm{Ni}$ catalyst poisoned with quinoline (ca. $1 \%)$ led to the expected tert-butyl substituted diene whereas only one of the two bonds was hydrogenated using the $\mathrm{Pd} /$ $\mathrm{BaSO}_{4}$ conditions. Although the conversion was complete, the desired diene was, however, collected in a poor yield only (38\%).

\subsection{Preference for a Zn-Mediated Procedure}

Using the Lindlar catalyst or the diimide reagent, Naf et al. failed $^{486}$ to obtain the $(1,3 Z, 5 Z)$-undecatriene from its corresponding 1,3( $Z$ )-diene-5-yne structure, owing to the lack of selectivity of these two reducing systems. Interestingly, the activated zinc powder gave the expected pure triene in good yield, although the reproducibility of this experiment proved poor (reaction incomplete sometimes). ${ }^{486}$

In contrast to Lindlar catalyst, the activated zinc system does not reduce simple, nonactivated triple bonds. On the other hand, with polyunsaturated structures, the activated zinc system works better than the other methods of hydrogenation. ${ }^{237,501}$ It was proved to be more reliable and more predictable. ${ }^{501}$
Avignon-Tropis dramatically improved ${ }^{483}$ the yield of the stereoselective reduction of a $(E, E)$-conjugated dienyne to its corresponding (E,E,Z)-conjugated triene (70\%) using activated $\mathrm{Zn}(\mathrm{Cu} / \mathrm{Ag})$ in methanol/water $(1 / 1)$ instead of the Lindlar hydrogenation, which afforded $30 \%$ only of the desired triene from a complex mixture. Attempts using hydrogenation over $\mathrm{Pd} / \mathrm{BaSO}_{4}$ or reduction with diisoamylborane were unsuccessful. $^{483}$

Chou et al. ${ }^{249}$ observed that catalytic hydrogenation with Lindlar catalyst or diimide reduction worked poorly with unprotected alcohol, and isolation of the expected target required tedious chromatography. In their hands, the Rieke zinc metal was found to be a superior reagent for effecting efficiently the partial reduction.

Bhatt et al. ${ }^{543}$ led to the same conclusion in their course to 3hydroxy leukotriene B4. They tried several reagents to convert a $(E, E)$-conjugated dienyne in the presence of an isolated $(Z)$ alkene to its corresponding (E,E,Z)-triene: whereas catalytic hydrogenation using P2-Ni or Lindlar catalyst was complicated by incomplete reaction and/or over-reduction, the semireduction using the Rieke zinc furnished the target $(E, E, Z)$ triene in $90 \%$ yield. In their hands, the zinc-copper couple as recommended by Avignon-Tropis ${ }^{483}$ was somewhat sluggish and never reached completion.

Interestingly, in the same synthetic route, Mohamed et al. ran a regioselective hydrogenation of a TMS-diyne with the Lindlar catalyst whereas the chemoselective hydrogenation of alkynes in the presence of other double bonds ( $E, E)$-diene and isolated $(Z)$-olefin, Scheme 58$)$ is achieved with the activated zinc reduction conditions. ${ }^{330}$

A range of reduction procedures were compared in terms of yields and stereoselectivity to determine the best reducing reagent for the preparation of 2-(Z)-alkenylthiophenes. ${ }^{544}$ Similar yields could be obtained using $\mathrm{Zn}(\mathrm{Cu} / \mathrm{Ag})$ reagent or Lindlar catalyst with slight deterioration in stereoselectivity for the latter $(Z / E$ ratio: $92 / 8$ and $91 / 3+6 \%$ over-reduction, respectively).

\subsection{Preference for Reduction via a Hydroboration Reaction}

Millar et al. ${ }^{503}$ found that reduction of skipped triynes with dicyclohexylborane gave better results than catalytic semihydrogenation, which is frequently beset with problems such as over-reduction, isomerization of the resulting double bonds, and catalytic poisoning with trace of impurities present in the starting substrate. Svatos report is in complete agreement with such comments, insisting on the good purity without tedious purification steps. $^{505}$

Similarly, Brudermüller et al. ${ }^{504}$ isolated an expected symmetrical skipped triene in $>94 \%$ isomeric purity $(72 \%$ yield), whereas they failed to produce a pure all-cis sample with the Lindlar catalyst system.

In addition, in the presence of a terminal alkene, selectivity was superior with hydroboration methodology rather than other reductions. ${ }^{411}$

Furthermore, it may be crucial in some cases to be sure that there is no $\mathrm{H}$-abstraction during the reduction or hydrogenation. While authors are confident with the hydroboration process, metal-mediated reduction tends to exchange hydrogens at the allylic position. ${ }^{511}$

The semireduction of a phenyldimethylsilyl-protected alkyne to its corresponding pure vinylsilane could be achieved with dicyclohexylborane (hydrolysis with $\mathrm{AcOH}$ ) only. ${ }^{305}$ Disappointing results were obtained with many other reducing 
systems, including DIBAL-H in THF at reflux (only $31 \%$ conversion), DIBAL-H (8/1: $Z / E$ and incomplete reaction), $\mathrm{Sia}_{2} \mathrm{BH}$ in THF (failed to provide the alkene), but also polymer-bound nickel boride (variable selectivities). In addition, hydrogenation over Lindlar or Rosenmund catalysts under various conditions also resulted in low and variable selectivities (2-5/1: $Z / E$ mixtures, over-reduction) and irreproductibility. $^{305}$

\subsection{Preference for a Ti-Mediated Reduction}

Lara-Ochoa et al. preferred the [titanium, $n$-BuLi] system, which gave more predictable yields and an easier purification method. $^{524}$ By contrast, partial hydrogenation provided a sample which is contaminated with a minor amount of the corresponding alkane, thus requiring tedious purification.

For deuterium incorporation, Kitching advised reduction of alkynes or poly ynes via the titanium isopropoxideisopropylmagnesium bromide system since, this methodology has the advantage to avoid any deuterium scrambling, unlike the heterogeneous catalysis. ${ }^{254}$ Working on deuteroboration of skipped diyne, Meyer et al. ${ }^{511}$ also pointed out that metalmediated reductions tend to exchange hydrogens at allylic positions. $^{511,545}$ This problem is particularly troublesome in isotope effects measured using scintillation and mass spectroscopy. $^{511}$

\subsection{Preferences for Tri(n-butyl)boron}

The use of tri(n-butyl)boron for the partial reduction of the symmetric undecadiyne was found to yield a purer sample than the one obtained with the Lindlar catalyst, or the diisoaamylborane. ${ }^{533}$

However, as mentioned in our above section (see section 3.7.2), the extremely high reaction temperature dramatically limits the application of this method to total synthesis of sensitive functionalized molecules.

\subsection{Preference for Rhodium Catalyst}

After several unfruitful Lindlar hydrogenations in different solvents, a bicyclic ynone was eventually converted to the expected $(Z)$-derivative using $\mathrm{Rh}(\mathrm{nbd})(\mathrm{dppb})] \mathrm{BF}_{4}$ in acetone. $^{150}$

\subsection{Preference for the $\mathrm{NiCl}_{2}-\mathrm{NaBH}_{4}$ System}

In order to overcome some problems of over-reduction and/or decomposition but also nonreproducibility with the Lindlar catalyst, the reducing system $\left[\mathrm{NiCl}_{2}, \mathrm{NaBH}_{4}\right]$ provided efficiently the desired $(Z)$-alkene based-modified nucleotides. ${ }^{122}$

\subsection{Preference for the Hydroalumination Reaction}

Meilert et al. ${ }^{530,546}$ encountered difficulties with the semireduction of a TMS-alkyne function. They obtained much better results with a hydroalumination reaction (DIBAL-H in ether, rt to reflux) than with semihydrogenations over Rosenmund catalyst (moderate E/Z-stereoselectivities and poor yields) or over Lindlar catalyst (variable selectivities). Marko et al. ${ }^{531}$ drew the same conclusion after many disappointing results with the Lindlar catalyst, which showed a moderate to bad $Z / E$ ratio $(8 / 1$ to $2 / 1)$.

\subsection{Preference for the Trost's Catalyst}

In the presence of a terminal alkene, the homogeneous hydrogen system using the catalyst $(\mathrm{dba})_{3} \mathrm{Pd}_{2} \cdot \mathrm{CHCl}_{3}(\mathrm{P}(o-$ toluyl $)_{3}, \mathrm{AcOH}$, hydrosilane) was shown to be more chemoselective $(52 \%,>20 / 1: Z / E)$ than the semihydrogenation over
Lindlar catalyst. The latter gave preferentially the reduction of the terminal double bond. ${ }^{286}$

\subsection{Sometimes Nothing Works}

Several reducing systems were studied by van Summeren et al. for the obtention of mycolactones $\mathrm{A}$ and $\mathrm{B} .{ }^{547}$ Lindlar and Brown catalysts, but also the $\mathrm{Zn} /(\mathrm{Cu} / \mathrm{Ag})$ couple, furnished over-reduced products only while no conversion was observed with the Elsevier's ${ }^{135}$ homogeneous catalyst (i.e., zerovalent palladium catalyst bearing bidentate nitrogen ligand). Their synthetic route remained unsuccessful.

\section{CONCLUSION}

In order to give hints to practitioners developing a retrosynthetic analysis, we first reported an overview of the different methods for alkyne semireductions, discussing their advantages and drawbacks (side reactions, stereoselectivity, function tolerance, commercial availability, convenience).

Many methods were only utilized in molecules which were poorly functionalized and/or did not contain any olefin.

The second part of this review focused on the methods applied in the total synthesis of natural or medicinal compounds, discussing their chemo-, regio-, and stereoselectivities.

Comparison of results obtained with different hydrogenation catalysts or reductive agents was seldom reported by authors. However, from the pieces of information that we could gather in section 4, it was shown that the Lindlar catalyst is the most frequently used catalyst, although it is also the most criticized one.

Most methods have been plagued by over-reduction or/and formation of $Z / E$-isomer mixtures (especially Lindlar, Rosenmund, Brown, and Trost methods) even though the desired $(Z)$-alkenes were usually obtained in a range of $>90 \%$ purity. Purification of such side-products was generally troublesome, requiring $\mathrm{AgNO}_{3}$-impregnated-silica gel or preparative HPLC. In some cases, the separation of the $Z, E$-mixture was unfortunately impossible.

Contrary to what is generally believed, none of the hydrogenation systems really halts at the stage of the generated $(Z)$-alkene. The rate of the reduction of alkenes and alkynes depends on their environment. Thus, in poly ynes, some alkyne functions may not react while some alkynes may be overreduced. If the conditions are not adapted (reaction time, temperature, equivalent of catalyst poisoning reagent), some double bonds (in situ generated or present in the starting material) may suffer from the hydrogenation reaction, whatever is the chosen catalyst.

The Ti-mediated process and hydroboration reaction together with the $\mathrm{Zn}$-activated system seem appealing, since they are much easier to set up than the hydrogenation reaction involving hydrogen gas. In addition, interestingly, no overreduction is observed. However, the Ti-mediated process and hydroboration reaction did not catch too much the chemists' attention, maybe because of the following: (a) the disappointing yields (rather weak in comparison with the ones obtained in Lindlar or Brown procedures; (b) the limitation of some functional groups' resistance; and (c) in the case of titanium(IV) isopropoxide, the significant risk of isomerization of the (Z)-skipped dienes. In addition, only a moderate chemoselectivity of the hydroboration reaction in the presence of other isolated or conjugated double bonds can be expected. 
Neither nonactivated skipped diyne nor any skipped poly yne was reported using the Zn-activated system. Actually, nonactivated triple bonds tend to react poorly only.

Incorporation of deuterium or tritium atoms was successfully attained with either the Lindlar, Rosenmund, or Brown catalysts but also the Zn-activated method, the Ti-mediated process, and the hydroboration reaction. Interestingly, the three latter showed no deuterium scrambling, and cheap reagents, including the convenient source of deuterium $\left(D_{2} \mathrm{O}\right)$.

Among all the herein reported methods, the Brown reaction procedure appeared to be the most tunable conditions to reach the best chemo-, regio-, and ( $Z$ )-stereoselectivities in polyfunctionalized bioactive molecules.

It appears difficult to predict which reducing system will be the more suitable for the desired target. Despite the impressive number of contributions and results obtained, it seems that it is always a challenge to succeed with alkyne reduction in the presence of other alkynes and/or alkenes.

\section{AUTHOR INFORMATION}

\section{Corresponding Author}

*E-mail: balas@univ-montp1.fr.

Notes

The authors declare no competing financial interest.

\section{Biographies}

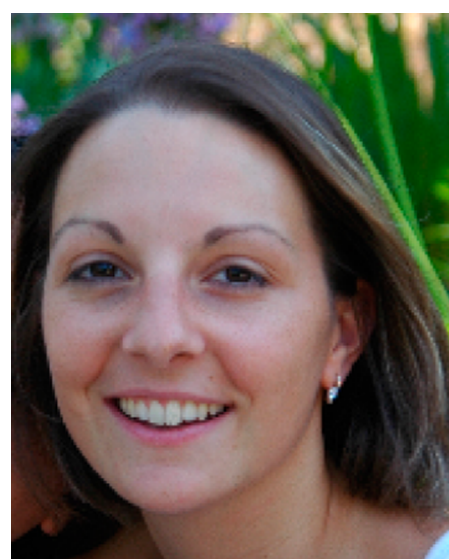

Camille Oger was born in Saint-Gaudens (France) in 1984. She studied chemistry at Paul Sabatier University in Toulouse and University of Paris VI. She then moved to Montpellier and received her Ph.D. in the field of total synthesis of neuroprostanes at the University of Montpellier II, in 2010, under the supervision of Dr. J.M. Galano and Dr. T. Durand. She did postdoctoral work with Pr. Ilan Marek at the Technion-Israel Institute of Technology on the development of new methodologies toward the synthesis of polypropionate units and was promoted as assistant professor at University of Montpellier I, in 2011. Her research interests include the development of new strategies toward the synthesis of bioactive lipids.

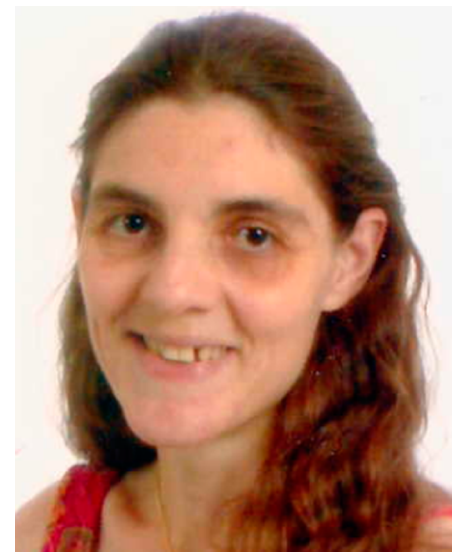

Laurence Balas studied chemistry at the University of Bordeaux and received her Ph.D. degree in 1992 from the University of Bordeaux under the direction of Prof. Joseph Vercauteren. After a postdoctoral fellowship on asymmetric radical chemistry under the supervision of Pr. Dennis P. Curran at the University of Pittsburgh in 1993, she joined the Centre National de la Recherche Scientifique (CNRS) in Nancy. She moved to the Laboratory of Bioactive Lipids at Institute of Biomolecules Max Mousseron (IBMM) in Montpellier in 1997. She is currently involved in the total synthesis of noncyclic metabolites of PUFAs (leukotrienes, endocannabinoids, ...) .

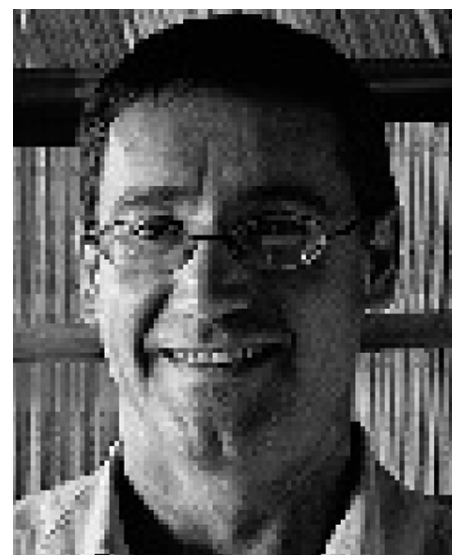

Thierry Durand studied chemistry at University of Paris VI and then moved to Montpellier and received his Ph.D. degree at University of Montpellier I in 1990. After postdoctoral training at Florida Institute of Technology in Melbourne, FL, USA, with Joshua Rokach, he became Chargé de Recherche CNRS at the University of Montpellier I in 1991. He finished his Habilitation in 1996 and became Directeur de Recherche CNRS in 2002. He is a group leader at the Institute of Biomolécules Max Mousseron. His research interests include the total synthesis of oxygenated cyclic and noncyclic metabolites of polyunsaturated fatty acids, mainly leukotrienes, iso-, phyto-, and neuroprostanes, as well as endocannabinoid analogs and the understanding of the role of such bioactive lipids by developing collaborations with chemists, biochemists, biologists, and clinicians over the word. 


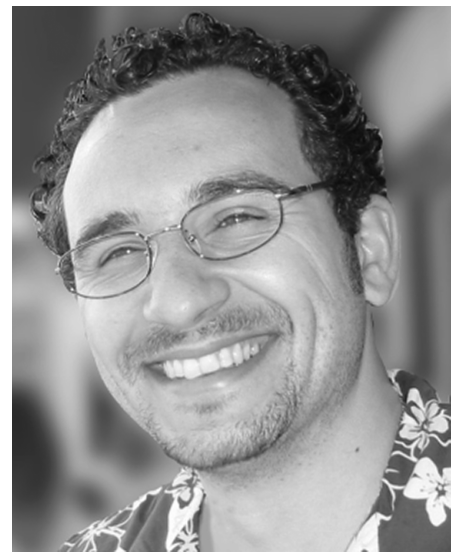

Jean-Marie Galano studied chemistry at Paul Cézanne Université Marseille and obtained his Ph.D. in the field of total synthesis under the supervision of Honore Monti in 2001. He then moved to the University of Oxford to pursue a postdoctoral fellowship with David $\mathrm{H}$. Hodgson on the development of new methods for the total synthesis of natural products. In October 2005 he joined the CNRS as a Chargé de Recherche at University of Montpellier I. His research focuses on new methods and strategies toward the total synthesis of natural products.

\section{ACKNOWLEDGMENTS}

We thank all the authors quoted in the references for their dedicated work in this field. We gratefully acknowledge the CNRS, the French Minister of Education and Research, the University of Montpellier I (Grants BQR 2008 and 2011), the PEPII INSB-INC, the Fondation pour la Recherche Médicale (DCM20111223047), and INRA (INRA-AlimH 2008-2010) for their financial support.

\section{LIST OF ABBREVIATIONS}

\begin{tabular}{|c|c|}
\hline BDMS & benzyldimethylsilyl \\
\hline brsm & based on ratio of starting material \\
\hline cy & cyclohexane \\
\hline $\mathrm{DAB}$ & diazabutadiene \\
\hline dba & dibenzylideneacetone \\
\hline DFT & density functional theory \\
\hline DMPS & dimethylphenylsilyl \\
\hline dppb & 1,4-bis(diphenylphosphino)butane \\
\hline eda & ethylenediamine \\
\hline $\mathrm{EE}$ & 1-ethoxyethyl \\
\hline equiv & equivalent \\
\hline IMes & 1,3-dimesitylimidazol-2-ylidene \\
\hline MA & maleic anhydride \\
\hline phtal & phthaloyl \\
\hline PMHS & polymethylhydrosiloxane \\
\hline PUFA & polyunsaturated fatty acid \\
\hline Pyr & pyridine \\
\hline rac & racemic \\
\hline rt & room temperature \\
\hline $\mathrm{Sia}_{2} \mathrm{BH}$ & diisoamylborane \\
\hline TBDPS & tert-butyldiphenylsilyl \\
\hline TEMPO & 2,2,6,6-tetramethylpiperidinoxyl \\
\hline TMS-alkyne & 1-(trimethylsilyl)-1-alkyne \\
\hline TMS-diyne & TMS-protected alkyne in a skipped diyne \\
\hline
\end{tabular}

\section{REFERENCES}

(1) Paust, J.; John, M. U.S. Patent US5689022, 1997.
(2) Nicolaou, K. C.; Daines, R. A.; Chakraborty, T. K.; Ogawa, Y. J. Am. Chem. Soc. 1987, 109, 2821.

(3) Semenov, V. V.; Kiselyov, A. S.; Titov, I. Y.; Sagamanova, I. K.; Ikizalp, N. N.; Chernysheva, N. B.; Tsyganov, D. V.; Konyushkin, L. D.; Firgang, S. I.; Semenov, R. V.; Karmanova, I. B.; Raihstat, M. M.; Semenova, M. N. J. Nat. Prod. 2010, 73, 1796.

(4) Chen, M.; Roush, W. R. Org. Lett. 2012, 14, 1880.

(5) Mori, K. In The total synthesis of natural products; ApSimon, J., Ed.; Wiley Interscience: New York, 1992; Vol. 9.

(6) Mori, K. Top. Curr. Chem. 2004, 239, 1.

(7) Odinokov, V. N. Chem. Nat. Compd. 2000, 36, 11.

(8) Fouché, M.; Rooney, L.; Barrett, A. G. M. J. Org. Chem. 2012, 77, 3060.

(9) Wittig, G.; Schollkopf, U. Chem. Ber. 1954, 87, 1318.

(10) Maryanoff, B. E.; Reitz, A. B. Chem. Rev. 1989, 89, 863.

(11) Wadsworth, W.; Emmons, W. D. J. Am. Chem. Soc. 1961, 83, 1733.

(12) Blakemore, P. R. J. Chem. Soc., Perkin Trans. 1 2002, 2563.

(13) Peterson, D. J. J. Org. Chem. 1968, 33, 780.

(14) Takai, K.; Nitta, K.; Utimoto, K. J. Am. Chem. Soc. 1986, 108, 7408.

(15) Keitz, B. K.; Endo, K.; Patel, P. R.; Herbert, M. B.; Grubbs, R. H. J. Am. Chem. Soc. 2012, 134, 693.

(16) Handbook of metathesis; Grubbs, R. H., Ed.; Wiley-CH: Weinheim, Germany, 2003.

(17) Trost, B. M.; Ball, Z. T.; Joge, T. J. Am. Chem. Soc. 2002, 124, 7922.

(18) Metal-catalyzed cross-coupling reactions; De Meijere, A.; Diederich, F., Eds.; Wiley-VCH: Weinheim, 2004.

(19) Furstner, A.; Radkowski, K. Chem. Commun. 2002, 2182.

(20) Munslow, I. In Modern reduction methods; Andersson, P. G., Munslow, I., Eds.; Wiley-VCH Verlag GmbH\&Co. KgaA: Weiheim, Germany, 2008; Chapter 15.

(21) March's advanced organic chemistry: reactions: mechanisms, and structure, 5th ed.; Smith, M. B., March, J., Eds.; Wiley-Interscience: New York, 2001.

(22) Organic chemistry; Ege, S., Ed.; D.C. Heath and Company: 1984.

(23) Handbook of heterogeneous catalytic hydrogenation for organic synthesis; Nishimura, S., Ed.; John Wiley and Sons: 2001.

(24) Kluwer, A. M.; Elsevier, C. J. In Handbook of homogeneous hydrogenation.; de Vries, J. G., Elsevier, C. J., Eds.; Wiley-VCH Verlag GmbH \& Co. KGaA: 2007; Chapter 14.

(25) Marvell, E. N.; Li, T. Synthesis 1973, 457.

(26) Molnar, A.; Sarkany, A.; Varga, M. J. Mol. Catal. A: Chem. 2001, 173,185 .

(27) Comprehensive organic synthesis. Selectivity, strategy and efficiency in Modern Organic Synthesis; Trost, B. M., Fleming, I., Eds.; Pergamon Press: Oxford, 1991; Vol. 8.

(28) Pacher, P.; Batkai, S.; Kunos, G. Pharmacol. Rev. 2006, 58, 389.

(29) Fride, E.; Gobshtis, N. Immunol., Endocr. Metab. Agents Med. Chem. 2007, 7, 157.

(30) Jahn, U.; Galano, J. M.; Durand, T. Angew. Chem., Int. Ed. Engl. 2008, 47, 5894.

(31) Roberts, L. J.; Fessel, J. P. Chem. Phys. Lipids 2004, 128, 173.

(32) Serhan, C. N.; Petasis, N. A. Chem. Rev. 2011, 111, 5922.

(33) Lindlar, H. Helv. Chim. Acta 1952, 35, 446.

(34) Lindlar, H.; Dubuis, R. Org. Synth. 1966, 46, 89.

(35) Ulan, J. G.; Kuo, E.; Maier, W. F.; Rai, R. S.; Thomas, G. J. Org. Chem. 1987, 52, 3126.

(36) Ulan, J. G.; Maier, W. F.; Smith, D. A. J. Org. Chem. 1987, 52, 3132.

(37) Steenhoe., A; Vanwijng., Bh; Pabon, H. J. J. Recl. Trav. Chim. Pays-Bas 1971, 90, 961.

(38) Garcia-Mota, M.; Gomez-Diaz, J.; Novell-Leruth, G.; VargasFuentes, C.; Bellarosa, L.; Bridier, B.; Perez-Ramirez, J.; Lopez, N. Theor. Chem. Acc. 2011, 128, 663.

(39) Verniest, G.; Boterberg, S.; Colpaert, J.; Van Thienen, T.; Stevens, C. V.; De Kimpe, N. Synlett 2004, 1273. 
(40) Handbook of organopalladium chemistry for organic chemistry; Negishi, E., Ed.; John Wiley \& Sons: New York, 2002; Vol. 2.

(41) McMurry, J. E.; Siemers, N. O. Tetrahedron Lett. 1994, 35, 4505.

(42) Hamprecht, D.; Josten, J.; Steglich, W. Tetrahedron 1996, 52, 10883.

(43) Knight, D. W.; Little, P. B. Tetrahedron Lett. 1998, 39, 5105.

(44) Naidu, S. V.; Gupta, P.; Kumar, P. Tetrahedron 2007, 63, 7624.

(45) Evans, D. A.; Fitch, D. M. Angew. Chem., Int. Ed. Engl. 2000, 39, 2536

(46) Rzasa, R. M.; Shea, H. A.; Romo, D. J. Am. Chem. Soc. 1998, $120,591$.

(47) Noyori, R.; Suzuki, M. Angew. Chem., Int. Ed. Engl. 1984, 23, 847.

(48) Zhang, Z. B.; Wang, Z. M.; Wang, Y. X.; Liu, H. Q.; Lei, G. X.; Shi, M. Tetrahedron: Asymmetry 1999, 10, 837.

(49) Guo, C.; Lu, X. Y. Tetrahedron Lett. 1991, 32, 7549.

(50) Nicolaou, K. C.; Xu, J. Y.; Kim, S.; Ohshima, T.; Hosokawa, S.; Pfefferkorn, J. J. Am. Chem. Soc. 1997, 119, 11353.

(51) Paterson, I.; Tudge, M. Tetrahedron 2003, 59, 6833.

(52) Gaudin, J. M.; Morel, C. Tetrahedron Lett. 1990, 31, 5749.

(53) Mcgaffin, G.; Demeijere, A. Synthesis 1994, 583.

(54) Wade, P. A.; DAmbrosio, S. G.; Rao, J. A.; ShahPatel, S.; Cole,

D. T.; Murray, J. K.; Carroll, P. J. J. Org. Chem. 1997, 62, 3671.

(55) Altenbach, H. J.; Himmeldirk, K. Tetrahedron: Asymmetry 1995, 6, 1077 .

(56) Campos, K. R.; Cai, D. W.; Journet, M.; Kowal, J. J.; Larsen, R. D.; Reider, P. J. J. Org. Chem. 2001, 66, 3634.

(57) Nowak, W.; Gerlach, H. Liebigs Ann. Chem. 1993, 153.

(58) Dobson, N. A.; Willis, R. G.; Eglinton, G.; Raphael, R. A.; Krishnamurthi, M. Tetrahedron 1961, 16, 16.

(59) Petrov, O. I.; Gerova, M. S.; Chanev, C. D.; Petrova, K. V. Synthesis 2011, 3711.

(60) Furst, R.; Zupko, I.; Berenyi, A.; Ecker, G. F.; Rinner, U. Bioorg. Med. Chem. Lett. 2009, 19, 6948.

(61) Hollingworth, G. J.; Richecoeur, A. M. E.; Sweeney, J. J. Chem. Soc., Perkin Trans. 1 1996, 2833.

(62) Zehnter, R.; Gerlach, H. Liebigs Ann. 1995, 2209.

(63) Srihari, P.; Bhasker, E. V.; Reddy, A. B.; Yadav, J. S. Tetrahedron Lett. 2009, 50, 2420.

(64) Zhou, W.-S.; Shen, Z.-W. J. Chem. Soc., Perkin Trans. 1 1991, 11, 2827.

(65) Miyaoka, H.; Hara, Y.; Shinohara, I.; Kurokawa, T.; Yamada, Y. Tetrahedron Lett. 2005, 46, 7945.

(66) Chen, S. Q.; Janda, K. D. J. Am. Chem. Soc. 1997, 119, 8724.

(67) Mangalagiu, I.; Benneche, T.; Undheim, K. Acta Chem. Scand. 1996, 50, 914.

(68) Bulman-page, P. C.; Ley, S. V. J. Chem. Soc., Perkin Trans. 1 1984, 1847.

(69) Compain, P.; Gore, J.; Vatele, J. M. Tetrahedron Lett. 1995, 36, 4063.

(70) Mcintosh, M. C.; Weinreb, S. M. J. Org. Chem. 1993, 58, 4823.

(71) Oppolzer, W.; Bochet, C. G. Tetrahedron: Asymmetry 2000, 11, 4761.

(72) Compain, P.; Gore, J.; Vatele, J. M. Tetrahedron 1996, 52, 6647.

(73) Chandrasekhar, S.; Narsihmulu, C.; Chandrashekar, G.; Shyamsunder, T. Tetrahedron Lett. 2004, 45, 2421.

(74) Sanders, T. C.; Hammond, G. B. J. Org. Chem. 1993, 58, 5598.

(75) Bennett, J. A.; Creamer, N. J.; Deplanche, K.; Macaskie, L. E.; Shannon, I. J.; Wood, J. Chem. Eng. Sci. 2010, 65, 282.

(76) Liprandi, D. A.; Cagnola, E. A.; Quiroga, M. E.; L’Argentiere, P. C. Catal. Lett. 2009, 128, 423.

(77) Nadkarni, D. V.; Fly, J. L. J. Chem. Soc., Chem. Commun 1993, 997.

(78) Marin-Astorga, N.; Pecchi, G.; Fierro, J. L. G.; Reyes, P. Catal. Lett. 2003, 91, 115.

(79) Choudary, B. M.; Kantam, M. L.; Reddy, N. M.; Rao, K. K.; Haritha, Y.; Bhaskar, V.; Figueras, F.; Tuel, A. Appl. Catal., A 1999, $181,139$.

(80) Mastalir, A.; Kiraly, Z. J. Catal. 2003, 220, 372.
(81) Tew, M. W.; Emerich, H.; van Bokhoven, J. A. J. Phys. Chem. C 2011, 115, 8457.

(82) Brunet, J. J.; Caubere, P. J. Org. Chem. 1984, 49, 4058.

(83) Yoon, N. M.; Park, K. B.; Lee, H. J.; Choi, J. Tetrahedron Lett. 1996, 37, 8527.

(84) Hori, J.; Murata, K.; Sugai, T.; Shinohara, H.; Noyori, R.; Arai, N.; Kurono, N.; Ohkuma, T. Adv. Synth. Catal. 2009, 351, 3143.

(85) Choudary, B. M.; Bharathi, P. J. Chem. Soc., Chem. Commun. 1987, 1505.

(86) Sharma, G. V. M; Choudary, B. M.; Sarma, M. R.; Rao, K. K. J. Org. Chem. 1989, 54, 2997.

(87) Choudary, B. M.; Vasantha, G.; Sharma, M.; Bharathi, P. Angew. Chem., Int. Ed. Engl. 1989, 28, 465.

(88) Gruttadauria, M.; Noto, R.; Deganello, G.; Liotta, L. F. Tetrahedron Lett. 1999, 40, 2857.

(89) Yermakov, Y. I. Catal. Rev. 1986, 13, 77.

(90) Gruttadauria, M.; Liotta, L. F.; Noto, R.; Deganello, G. Tetrahedron Lett. 2001, 42, 2015.

(91) Guczi, L.; Schay, Z.; Stefler, G.; Liotta, L. F.; Deganello, G.; Venezia, A. M. J. Catal. 1999, 182, 456.

(92) Sajiki, H.; Hattori, K.; Hirota, K. J. Org. Chem. 1998, 63, 7990.

(93) Kurosawa, K.; Nagase, T.; Chida, N. Chem. Commun. 2002, 1280.

(94) Kurosawa, K.; Matsuura, K.; Chida, N. Tetrahedron Lett. 2005, 46, 389.

(95) Sajiki, H.; Hattori, K.; Hirota, K. J. Chem. Soc., Perkin Trans. 1 1998, 4043.

(96) Sajiki, H.; Hattori, K.; Hirota, K. Chem. Commun. 1999, 1041.

(97) Tahirovic, Y. A.; Geballe, M.; Gruszecka-Kowalik, E.; Myers, S. J.; Lyuboslavsky, P.; Le, P.; French, A.; Irier, H.; Choi, W. B.; Easterling, K.; Yuan, H.; Wilson, L. J.; Kotloski, R.; McNamara, J. O.; Dingledine, R.; Liotta, D. C.; Traynelis, S. F.; Snyder, J. P. J. Med. Chem. 2008, 51, 5506.

(98) Sajiki, H.; Hattori, K.; Hirota, K. Chem.-Eur. J. 2000, 6, 2200. (99) Hattori, K.; Sajiki, H.; Hirota, K. Tetrahedron 2000, 56, 8433.

(100) Oozeki, H.; Tajima, R; Nihei, K. Bioorg. Med. Chem. Lett. 2008, 18, 5252 .

(101) Sajiki, H.; Mori, S.; Ohkubo, T.; Ikawa, T.; Kume, A.; Maegawa, T.; Monguchi, Y. Chem.-Eur. J. 2008, 14, 5109.

(102) Moroni, M.; Koksch, B.; Osipov, S. N.; Crucianelli, M.; Frigerio, M.; Pierfrancesco, B.; Burger, K. J. Org. Chem. 2001, 66, 130.

(103) Mori, S.; Ohkubo, T.; Ikawa, T.; Kume, A.; Maegawa, T.; Monguchi, Y.; Sajiki, H. J. Mol. Catal. A: Chem. 2009, 307, 77.

(104) Nijhuis, T. A.; vanKoten, G.; Moulijn, J. A. Appl. Catal., A 2003, 238, 259.

(105) Papp, A.; Molnar, A.; Mastalir, A. Appl. Catal., A 2005, 289, 256.

(106) Teschner, D.; Vass, E.; Havecker, M.; Zafeiratos, S.; Schnorch, P.; Sauer, H.; Knop-Gericke, A.; Schloegl, R.; Chamam, M.; Wootsch, A.; Canning, A. S.; Gamman, J. J.; Jackson, S. D.; McGregor, J.; Gladden, L. F. J. Catal. 2006, 242, 26.

(107) Belykh, L. B.; Goremyka, T. V.; Skripov, N. I.; Umanets, V. A.; Shmidt, F. K. Kinet. Catal. 2006, 47, 367.

(108) Semagina, N.; Grasemann, M.; Xanthopoulos, N.; Renken, A.; Kiwi-Minsker, L. J. Catal. 2007, 251, 213.

(109) Semagina, N.; Kiwi-Minsker, L. Catal. Lett. 2009, 127, 334.

(110) Schlesinger, H. I.; Brown, H. C.; Finholt, A. E.; Gilbreath, J. R.; Hoekstra, H. R.; Hyde, E. K. J. Am. Chem. Soc. 1953, 75, 215.

(111) P1 Ni and P2 Ni designations derived from Purdue University, where this work was initiated.

(112) Brown, C. A.; Brown, H. C. J. Am. Chem. Soc. 1963, 85, 1003.

(113) Brown, C. A. J. Chem. Soc., Chem. Commun. 1969, 952.

(114) Brown, H. C.; Brown, C. A. J. Am. Chem. Soc. 1963, 85, 1005.

(115) Brown, C. A. J. Org. Chem. 1970, 35, 1900.

(116) Brown, C. A.; Ahuja, V. K. J. Chem. Soc., Chem. Commun. 1973, 553.

(117) Peng, S.; McGinley, C. M.; van der Donk, W. A. Org. Lett. 2004, 6, 349. 
(118) Prakash, C.; Saleh, S.; Sweetman, B. J.; Taber, D. F.; Blair, I. A. J. Labelled Compd. Radiopharm. 1989, 27, 539.

(119) Hansen, T. V.; Stenstrom, Y. Tetrahedron: Asymmetry 2001, 12, 1407.

(120) Ganame, D.; Quach, T.; Poole, C.; Rizzacasa, M. A. Tetrahedron Lett. 2007, 48, 5841.

(121) Chemin, D.; Alami, M.; Linstrumelle, G. Tetrahedron Lett. 1992, 33, 2681.

(122) Lee, S. E.; Vyle, J. S.; Williams, D. M.; Grasby, J. A. Tetrahedron Lett. 2000, 41, 267.

(123) Feutrill, J. T.; Lilly, M. J.; White, J. M.; Rizzacasa, M. A. Tetrahedron 2008, 64, 4880.

(124) Han, L. N.; Razdan, R. K. Tetrahedron Lett. 1998, 39, 771.

(125) Savoia, D.; Tagliavini, E.; Trombini, C.; Umanironchi, A. J. Org. Chem. 1981, 46, 5340.

(126) Savoia, D.; Tagliavini, E.; Trombini, C.; Umanironchi, A. J. Org. Chem. 1981, 46, 5344.

(127) Brunet, J. J.; Gallois, P.; Caubere, P. Tetrahedron Lett. 1977, 3955.

(128) Yoon, N. M.; Choi, J. S. Synlett 1993, 135.

(129) Yoon, N. M.; Lee, H. J.; Ahn, J. H.; Choi, J. S. J. Org. Chem. 1994, 59, 4687.

(130) Yoon, N. M.; Choi, J. S.; Shon, Y. S. Synth. Commun. 1993, 23, 3047.

(131) Choi, J. S.; Yoon, N. M. Tetrahedron Lett. 1996, 37, 1057.

(132) Elsevier, C. J. Coord. Chem. Rev. 1999, 185-6, 809.

(133) van Laren, M. W.; Duin, M. A.; Klerk, C.; Naglia, M.; Rogolino, D.; Pelagatti, P.; Bacchi, A.; Pelizzi, C.; Elsevier, C. J. Organometallics 2002, 21, 1546.

(134) Kluwer, A. M.; Koblenz, T. S.; Jonischkeit, T.; Woelk, K.; Elsevier, C. J. J. Am. Chem. Soc. 2005, 127, 15470.

(135) van Laren, M. W.; Elsevier, C. J. Angew. Chem., Int. Ed. Engl. 1999, 38, 3715.

(136) Bacchi, A.; Carcelli, M.; Costa, M.; Pelagatti, P.; Pelizzi, C.; Pelizzi, G. J. Chem. Soc., Dalton Trans. 1996, 4239.

(137) Bacchi, A.; Carcelli, M.; Costa, M.; Leporati, A.; Leporati, E.; Pelagatti, P.; Pelizzi, C.; Pelizzi, G. J. Organomet. Chem. 1997, 535, 107.

(138) Pelagatti, P.; Venturini, A.; Leporati, A.; Carcelli, M.; Costa, M.; Bacchi, A.; Pelizzi, G.; Pelizzi, C. J. Chem. Soc., Dalton Trans. 1998, 2715.

(139) Costa, M.; Pelagatti, P.; Pelizzi, C.; Rogolino, D. J. Mol. Catal. A: Chem. 2002, 178, 21.

(140) Santra, P. K.; Sagar, P. J. Mol. Catal. A: Chem. 2003, 197, 37. (141) Albers, M. O.; Singleton, E.; Viney, M. M. J. Mol. Catal. 1985, 30, 213.

(142) Ashworth, T. V.; Nolte, M. J.; Singleton, E. J. Chem. Soc., Dalton Trans. 1977, 1816.

(143) Nkosi, B. S.; Coville, N. J.; Albers, M. O.; Singleton, E. J. Mol. Catal. 1987, 39, 313.

(144) Ashworth, T. V.; Chalmers, A. A.; Meintjies, E.; Oosthuizen, H. E.; Singleton, E. Organometallics 1984, 3, 1485.

(145) Lough, A. J.; Morris, R. H.; Ricciuto, L.; Schleis, T. Inorg. Chim. Acta 1998, 270, 238.

(146) Sodeoka, M.; Shibasaki, M. J. Org. Chem. 1985, 50, 1147.

(147) Sodeoka, M.; Shibasaki, M. Synthesis 1993, 643.

(148) Vasil'ev, A. A.; Serebryakov, E. P. Russ. Chem. Bull., Int. Ed. 2002, 51, 1341.

(149) Schrock, R. R.; Osborn, J. A. J. Am. Chem. Soc. 1976, 98, 2143.

(150) Nicolaou, K. C.; Xu, J. Y.; Kim, S.; Pfefferkorn, J.; Ohshima, T.; Vourloumis, D.; Hosokawa, S. J. Am. Chem. Soc. 1998, 120, 8661.

(151) Nicolaou, K. C.; Kim, S. H.; Pfefferkorn, J.; Xu, J. Y.; Ohshima, T.; Hosokawa, S.; Vourloumis, D.; Li, T. H. Angew. Chem., Int. Ed. Engl. 1998, 37, 1418.

(152) Sagar, P.; Rani, V. Synth. React. Inorg., Met.-Org., Nano-Met. Chem. 2010, 40, 754.

(153) de Wolf, E.; Spek, A. L.; Kuipers, B. W. M.; Philipse, A. P.; Meeldijk, J. D.; Bomans, P. H. H.; Frederik, P. M.; Deelman, B. J.; van Koten, G. Tetrahedron 2002, 58, 3911.
(154) Leitmannova, E.; Svoboda, J.; Sedlacek, J.; Vohlidal, J.; Kacer, P.; Cerveny, L. Appl. Catal., A 2010, 372, 34.

(155) Burk, M. J.; Gerlach, A.; Semmeril, D. J. Org. Chem. 2000, 65, 8933.

(156) Esteruelas, M. A.; Gonzalez, I.; Herrero, J.; Oro, L. A. J. Organomet. Chem. 1998, 551, 49.

(157) Gianetti, T. L.; Tomson, N. C.; Arnold, J.; Bergman, R. G. J. Am. Chem. Soc. 2011, 133, 14904.

(158) La Pierre, H. S.; Arnold, J.; Toste, F. D. Angew. Chem., Int. Ed. Engl. 2011, 50, 3900.

(159) Sgoutas, D. S.; Sanders, H.; Yang, E. M. J. Lipid Res. 1969, 10, 642.

(160) Brown, H. C.; Zweifel, G. J. Am. Chem. Soc. 1959, 81, 1512.

(161) Brown, H. C. Tetrahedron 1961, 12, 117.

(162) Brown, H. C.; Zweifel, G. J. Am. Chem. Soc. 1961, 83, 3834.

(163) Zweifel, G.; Brown, H. C. J. Am. Chem. Soc. 1963, 85, 2066.

(164) Brown, H. C.; Scouten, C. G.; Liotta, R. J. Am. Chem. Soc. 1979, 101, 96.

(165) Brown, H. C.; Campbell, J. B. J. Org. Chem. 1980, 45, 389.

(166) Brown, H. C.; Basavaiah, D.; Kulkarni, S. U. J. Organomet. Chem. 1982, 225, 63.

(167) Brown, H. C.; Basavaiah, D.; Kulkarni, S. U.; Bhat, N. G.; Prasad, J. V. N. V. J. Org. Chem. 1988, 53, 239.

(168) Brown, H. C.; Molander, G. A. J. Org. Chem. 1986, 51, 4512.

(169) Cha, J. S. Bull. Korean Chem. Soc. 2011, 32, 1808.

(170) Cha, J. S.; Min, S. J.; Kim, J. M.; Kwon, O. O. Tetrahedron Lett. 1994, 35, 2.

(171) Cha, J. S.; Min, S. J.; Kim, J. M.; Kwon, O. O. Tetrahedron Lett. 1993, 34, 5113.

(172) Pereira, S.; Srebnik, M. Tetrahedron Lett. 1996, 37, 3283.

(173) Beletskaya, I.; Pelter, A. Tetrahedron 1997, 53, 4957.

(174) Gridnev, I. D.; Miyaura, N.; Suzuki, A. Organometallics 1993, 12,589 .

(175) Iwadate, N.; Suginome, M. Org. Lett. 2009, 11, 1899.

(176) Konno, T.; Chae, J. H.; Tanaka, T.; Ishihara, T.; Yamanaka, H. J. Fluorine Chem. 2006, 127, 36.

(177) Jang, H.; Zhugralin, A. R.; Lee, Y.; Hoveyda, A. H. J. Am. Chem. Soc. 2011, 133, 7859.

(178) Wang, Y. N. D.; Kimball, G.; Prashad, A. S.; Wang, Y. Tetrahedron Lett. 2005, 46, 8777.

(179) Pereira, S.; Srebnik, M. Organometallics 1995, 14, 3127.

(180) Leyva, A.; Zhang, X.; Corma, A. Chem. Commun. 2009, 4947.

(181) Hartwig, J. F.; Muhoro, C. N. Organometallics 2000, 19, 30.

(182) Brown, H. C.; Wang, K. K. J. Org. Chem. 1986, 51, 4514.

(183) Bui, V. P.; Hudlicky, T.; Hansen, T. V.; Stenstrom, Y. Tetrahedron Lett. 2002, 43, 2839.

(184) Lawrence, N. J.; Ghani, F. A.; Hepworth, L. A.; Hadfield, J. A.; McGown, A. T.; Pritchard, R. G. Synthesis 1999, 1656.

(185) Odlo, K.; Klaveness, J.; Rongved, P.; Hansen, T. V. Tetrahedron Lett. 2006, 47, 1101.

(186) Mannig, D.; Noth, H. Angew. Chem., Int. Ed. Engl. 1985, 24, 878.

(187) Periasamy, M.; Prasad, A. S. B.; Suseela, Y. Tetrahedron 1995, 51, 2743.

(188) Soderquist, J. A.; Santiago, B. Tetrahedron Lett. 1990, 31, 5113.

(189) Yoshida, T.; Negishi, E. J. Chem. Soc., Chem. Commun. 1974, 762.

(190) Ashby, E. C.; Lin, J. J.; Goel, A. B. J. Org. Chem. 1978, 43, 757.

(191) Ryu, I.; Kusumoto, N.; Ogawa, A.; Kambe, N.; Sonoda, N. Organometallics 1989, 8, 2279.

(192) Daeuble, J. F.; Mcgettigan, C.; Stryker, J. M. Tetrahedron Lett. 1990, 31, 2397.

(193) Koenig, T. M.; Daeuble, J. F.; Brestensky, D. M.; Stryker, J. M. Tetrahedron Lett. 1990, 31, 3237.

(194) Miao, R.; Li, S. L.; Chiu, P. Tetrahedron 2007, 63, 6737.

(195) Leung, L. T.; Leung, S. K.; Chiu, P. Org. Lett. 2005, 7, 5249.

(196) Mahoney, W. S.; Stryker, J. M. J. Am. Chem. Soc. 1989, 111, 8818. 
(197) Mahoney, W. S.; Brestensky, D. M.; Stryker, J. M. J. Am. Chem. Soc. 1988, 110, 291

(198) Brestensky, D. M.; Stryker, J. M. Tetrahedron Lett. 1989, 30, 5677

(199) Tao, C. L.; Donaldson, W. A. J. Org. Chem. 1993, 58, 2134.

(200) Lipshutz, B. H.; Chrisman, W.; Noson, K.; Papa, P.; Sclafani, J. A.; Vivian, R. W.; Keith, J. M. Tetrahedron 2000, 56, 2779.

(201) Gallagher, B. M.; Zhao, H. J.; Pesant, M.; Fang, F. G. Tetrahedron Lett. 2005, 46, 923.

(202) Brummond, K. M.; Lu, J. L. J. Am. Chem. Soc. 1999, 121, 5087.

(203) Deutsch, C.; Krause, N. Chem. Rev. 2008, 108, 2916.

(204) Deutsch, C.; Lipshutz, B. H.; Krause, N. Angew. Chem., Int. Ed. Engl. 2007, 46, 1650.

(205) Chiu, P.; Li, Z. N.; Fung, K. C. M. Tetrahedron Lett. 2003, 44, 455.

(206) Diez-Gonzalez, S.; Nolan, S. P. Acc. Chem. Res. 2008, 41, 349.

(207) Mankad, N. P.; Laitar, D. S.; Sadighi, J. P. Organometallics 2004, 23, 3369.

(208) Ashby, E. C.; Noding, S. A. J. Org. Chem. 1980, 45, 1035.

(209) Eisch, J. J.; Kaska, W. C. J. Am. Chem. Soc. 1966, 88, 2213.

(210) Parenty, A.; Campagne, J. M. Tetrahedron Lett. 2002, 43, 1231.

(211) Petry, N.; Parenty, A.; Campagne, J. M. Tetrahedron: Asymmetry 2004, 15, 1199.

(212) Rossi, R.; Carpita, A. Synthesis 1977, 561.

(213) Lee, H. S. Bull. Korean Chem. Soc. 1987, 8, 484.

(214) Ashby, E. C.; Noding, S. R. J. Organomet. Chem. 1979, 177, 117.

(215) Chum, P. W.; Wilson, S. E. Tetrahedron Lett. 1976, 15.

(216) Gao, Y.; Sato, F. J. Chem. Soc., Chem. Commun. 1995, 659.

(217) Gao, Y.; Harada, K.; Hata, T.; Urabe, H.; Sato, F. J. Org. Chem. 1995, 60, 290.

(218) Handbook of reagents for organic synthesis-Oxidizing and reducing agents; Burke, S. D., Danheiser, R. L., Eds.; John Willey and Sons Ltd: 2000.

(219) Ashby, E. C.; Noding, S. A. J. Org. Chem. 1979, 44, 4364.

(220) Ibragimov, A. G.; Ramazanov, I. R.; Khalilov, L. M.; Sultanov, R. M.; Dzhemilev, U. M. Mendeleev Commun. 1996, 231.

(221) Ashby, E. C.; Lin, J. J. J. Org. Chem. 1978, 43, 2567.

(222) Gao, Y. A.; Urabe, H.; Sato, F. J. Org. Chem. 1994, 59, 5521.

(223) Sato, F.; Ishikawa, H.; Sato, M. Tetrahedron Lett. 1981, $22,85$.

(224) Sato, F. J. Organomet. Chem. 1985, 285, 53.

(225) Zhao, H.; Hao, W. Y.; Cai, M. Z.; Zhou, Z. J. Chem. Res., Synop. 2003, 780 .

(226) Eisch, J. J.; Galle, J. E. J. Organomet. Chem. 1978, 160, C8.

(227) Tanaka, K.; Kamatani, M.; Mori, H.; Fujii, S.; Ikeda, K.; Hisada, M.; Itagaki, Y.; Katsumura, S. Tetrahedron 1999, 55, 1657.

(228) Kocienski, P. J.; Love, C. J.; Whitby, R. J.; Costello, G.; Roberts, D. A. Tetrahedron 1989, 45, 3839.

(229) Alonso, F.; Radivoy, G.; Yus, M. Russ. Chem. Bull., Int. Ed. 2003, 52, 2563.

(230) Alonso, F.; Yus, M. Chem. Soc. Rev. 2004, 33, 284.

(231) Alonso, F.; Riente, P.; Yus, M. Acc. Chem. Res. 2011, 44, 379.

(232) Alonso, F.; Yus, M. Tetrahedron Lett. 1996, 37, 6925.

(233) Alonso, F.; Yus, M. Tetrahedron Lett. 1997, 38, 149.

(234) Alonso, F.; Calvino, J. J.; Osante, I.; Yus, M. Chem. Lett. 2005, $34,1262$.

(235) Alonso, F.; Osante, M.; Yus, M. Adv. Synth. Catal. 2006, 348, 305.

(236) Aerssens, M. H. P. J.; Brandsma, L. J. Chem. Soc., Chem. Commun. 1984, 735.

(237) Boland, W.; Sieler, C.; Feigel, M. Helv. Chim. Acta 1987, 70, 1025.

(238) Smith, R. D.; Simmons, H. E. Org. Synth. 1961, 41, 72.

(239) Sondengam, B. L.; Charles, G.; Akam, T. M. Tetrahedron Lett. 1980, 21, 1069.

(240) Treilhou, M.; Fauve, A.; Pougny, J. R.; Prome, J. C.; Veschambre, H. J. Org. Chem. 1992, 57, 3203.

(241) Solladie, G.; Stone, G. B.; Rubio, A. Tetrahedron Lett. 1993, 34, 1803.
(242) Borhan, B.; Souto, M. L.; Um, J. M.; Zhou, B. S.; Nakanishi, K. Chem.-Eur. J. 1999, 5, 1172.

(243) Boland, W.; Pantke, S. J. Prakt. Chem./Chem.-Ztg. 1994, 336, 714.

(244) Chemin, D.; Linstrumelle, G. Synthesis 1993, 377.

(245) Alami, M.; Crousse, B.; Linstrumelle, G.; Mambu, L.; Larcheveque, M. Tetrahedron: Asymmetry 1997, 8, 2949.

(246) Rodriguez, A. R.; Spur, B. W. Tetrahedron Lett. 2001, 42, 6057.

(247) Quesada, E.; Acuna, A. U.; Amat-Guerri, F. Eur. J. Org. Chem. 2003, 1308.

(248) Ekong, D. E. U.; Okogun, J. I.; Sondengam, B. L. J. Chem. Soc., Perkin Trans. 1 1975, 2118.

(249) Chou, W. N.; Clark, D. L.; White, J. B. Tetrahedron Lett. 1991, $32,299$.

(250) Rieke, R. D.; Hanson, M. V. Tetrahedron 1997, 53, 1925.

(251) Druais, V.; Hall, M. J.; Corsi, C.; Wendeborn, S. V.; Meyer, C.; Cossy, J. Org. Lett. 2009, 11, 935.

(252) Harada, K.; Urabe, H.; Sato, F. Tetrahedron Lett. 1995, 36, 3203.

(253) Hungerford, N. L.; Kitching, W. Chem. Commun. 1996, 1697. (254) Hungerford, N. L.; Kitching, W. J. Chem. Soc., Perkin Trans. 1 1998, 1839.

(255) Chow, S.; Kitching, W. Chem. Commun. 2001, 1040.

(256) Chow, S.; Kitching, W. Tetrahedron: Asymmetry 2002, 13, 779.

(257) Dickson, D. P.; Toh, C.; Lunda, M.; Yermolina, M. V.; Wardrop, D. J.; Landrie, C. L. J. Org. Chem. 2009, 74, 9535.

(258) Pasto, D. J. In Organic Reactions; Paquette, L. A., Ed.; John Wiley \& Sons: New York, 1991; Vol. 40; pp 91-155.

(259) Stereochemistry conformation and mechanism; 6th ed.; Kalsi, P. S., Ed.; New Age International Pvt Ltd Publishers: 2006.

(260) Cusack, N. J.; Reese, C. B.; Risius, A. C.; Roozpeikar, B. Tetrahedron 1976, 32, 2157.

(261) Moriarty, R. M.; Vaid, R. K.; Duncan, M. P. Synth. Commun. 1987, 17, 703.

(262) Hamersma, J. W.; Snyder, E. I. J. Org. Chem. 1965, 30, 3985.

(263) Marsh, B. J.; Carbery, D. R. J. Org. Chem. 2009, 74, 3186.

(264) Garbisch, E. W.; Schildcr, S. M.; Patterso, D. B.; Sprecher, C. M. J. Am. Chem. Soc. 1965, 87, 2932.

(265) Tang, H. R.; Mckee, M. L.; Stanbury, D. M. J. Am. Chem. Soc. 1995, 117, 8967.

(266) Smit, C.; Fraaije, M. W.; Minnaard, A. J. J. Org. Chem. 2008, $73,9482$.

(267) Lorenc, L.; Bondarenko-Gheorghiu, L.; Mihailovic, M. L. J. Chem. Res., Synop. 1998, 102.

(268) Mascitti, V.; Corey, E. J. J. Am. Chem. Soc. 2004, 126, 15664.

(269) Buszek, K. R.; Brown, N. J. Org. Chem. 2007, 72, 3125.

(270) Lacombe, P.; Castagner, B.; Gareau, Y.; Ruel, R. Tetrahedron Lett. 1998, 39, 6785.

(271) Palmer, C. J.; Casida, J. E. J. Labelled Compd. Radiopharm. 1991, 29, 829.

(272) Kwon, E.; Sakamoto, K.; Kabuto, C.; Kira, M. Chem. Lett. 2000, 1416.

(273) Corey, E. J.; Pasto, D. J.; Mock, W. L. J. Am. Chem. Soc. 1961, 83, 2957.

(274) Pasto, D. J.; Chipman, D. M. J. Am. Chem. Soc. 1979, 101, 2290.

(275) Johnstone, R. A. W.; Wilby, A. H.; Entwistle, I. D. Chem. Rev. 1985, 85, 129.

(276) The handbook of homogenous hydrogenation; de Vries, J. G., Elsevier, C. J., Eds.; Wiley-VCH Verlag GmbH \& Co. KGaA: Weinheim, 2007.

(277) Kang, S. K.; Park, D. C.; Cho, D. G.; Chung, J. U.; Jung, K. Y. J. Chem. Soc., Perkin Trans. 1 1994, 237.

(278) Tani, K.; Ono, N.; Okamoto, S.; Sato, F. J. Chem. Soc., Chem. Commun. 1993, 386.

(279) Hauwert, P.; Maestri, G.; Sprengers, J. W.; Catellani, M.; Elsevier, C. J. Angew. Chem., Int. Ed. Engl. 2008, 47, 3223. 
(280) Campana, A. G.; Estevez, R. E.; Fuentes, N.; Robles, R.; Cuerva, J. M.; Bunuel, E.; Cardenas, D.; Oltra, J. E. Org. Lett. 2007, 9, 2195.

(281) Solladie, G.; Stone, G. B.; Hamdouchi, C. Tetrahedron Lett. 1993, 34, 1807.

(282) Wei, L. L.; Wei, L. M.; Pan, W. B.; Leou, S. P.; Wu, M. J. Tetrahedron Lett. 2003, 44, 1979.

(283) Trost, B. M.; Braslau, R. Tetrahedron Lett. 1989, 30, 4657.

(284) Tsuji, J.; Minami, I.; Shimizu, I. Synthesis 1986, 623.

(285) Gladiali, S.; Alberico, E. Chem. Soc. Rev. 2006, 35, 226.

(286) Trost, B. M.; O’Boyle, B. M.; Hund, D. J. Am. Chem. Soc. 2009, $131,15061$.

(287) Padwa, A.; Bobeck, D. R.; Mmutlane, E. M. Arkivoc 2010, 7. (288) Bobeck, D. R.; Lee, H. I.; Flick, A. C.; Padwa, A. J. Org. Chem. 2009, 74, 7389.

(289) Kini, A. D.; Nadkarni, D. V.; Fry, J. L. Tetrahedron Lett. 1994, 35, 1507.

(290) Weir, J. R.; Patel, B. A.; Heck, R. F. J. Org. Chem. 1980, 45, 4926.

(291) Zhao, Y. K.; Liu, Q. A.; Li, J.; Liu, Z. Q.; Zhou, B. Synlett 2010, 1870.

(292) Hauwert, P.; Boerleider, R.; Warsink, S.; Weigand, J. J.; Elsevier, C. J. J. Am. Chem. Soc. 2010, 132, 16900.

(293) Warsink, S.; Hauwert, P.; Siegler, M. A.; Spek, A. L.; Elsevier, C. J. Appl. Organomet. Chem. 2009, 23, 225.

(294) Warsink, S.; Bosman, S.; Weigand, J. J.; Elsevier, C. J. Appl. Organomet. Chem. 2011, 25, 276.

(295) Li, J.; Hua, R.; Liu, T. J. Org. Chem. 2010, 75, 2966.

(296) Yamada, H.; Aoyagi, S.; Kibayashi, C. Tetrahedron Lett. 1996, 37, 8787.

(297) Shen, R. W.; Chen, T. Q.; Zhao, Y. L.; Qiu, R. H.; Zhou, Y. B.; Yin, S. F.; Wang, X. B.; Goto, M.; Han, L. B. J. Am. Chem. Soc. 2011, $133,17037$.

(298) Belger, C.; Neisius, N. M.; Plietker, B. Chem.-Eur. J. 2010, 16, 12214.

(299) Blum, Y.; Czarkie, D.; Rahamim, Y.; Shvo, Y. Organometallics $1985,4,1459$.

(300) Shvo, Y.; Goldberg, I.; Czerkie, D.; Reshef, D.; Stein, Z. Organometallics 1997, 16, 133.

(301) Gao, Y.; Jennings, M. C.; Puddephatt, R. J. Can. J. Chem. 2001, 79, 915 .

(302) Li, J.; Hua, R. Chem.-Eur. J. 2011, 17, 8462.

(303) Chung, S. K. J. Org. Chem. 1979, 44, 1014.

(304) Tani, K.; Iseki, A.; Yamagata, T. Chem. Commun. 1999, 1821.

(305) Denmark, S. E.; Herbert, B. J. Org. Chem. 2000, 65, 2887.

(306) Durand-Meulien, S. Ph.D. Dissertation, Université d'AixMarseille, 1998.

(307) Adams, J.; Rokach, J. Tetrahedron Lett. 1984, 25, 35.

(308) Feutrill, J. T.; Rizzacasa, M. A. Aust. J. Chem. 2003, 56, 783.

(309) Cossy, J.; Aclinou, P. Tetrahedron Lett. 1990, 31, 7615.

(310) Yasumoto, K.; Nishigami, A.; Yasumoto, M.; Kasai, F.; Okada, Y.; Kusumi, T.; Ooi, T. Tetrahedron Lett. 2005, 46, 4765.

(311) Kerdesky, F. A. J.; Schmidt, S. P.; Holms, J. H.; Dyer, R. D.; Carter, G. W.; Brooks, D. W. J. Med. Chem. 1987, 30, 1177.

(312) Lysek, R.; Kaluza, Z.; Furman, B.; Chmielewski, M. Tetrahedron 1998, 54, 14065.

(313) Bell, T. W.; Ciaccio, J. A. J. Org. Chem. 1993, 58, 5153.

(314) Hangyou, M.; Ishiyama, H.; Takahashi, Y.; Kubota, T.; Kobayashi, J. Tetrahedron Lett. 2009, 50, 1475.

(315) Mel'nikova, V. I.; Vasil'eva, L. L.; Pivnitsky, K. K. Russ. Chem. Bull., Int. Ed. 1998, 47, 1199.

(316) Ivanov, I. V.; Groza, N. V.; Romanov, S. G.; Kuhn, H.; Myagkova, G. I. Synthesis 2000, 691.

(317) Groza, N. V.; Ivanov, I. V.; Romanov, S. G.; Myagkova, G. I.; Nigam, S. Tetrahedron 2002, 58, 9859.

(318) Kigoshi, H.; Shizuri, Y.; Niwa, H.; Yamada, K. Tetrahedron Lett. 1981, 22, 4729.

(319) Du, Y.; Zheng, J. F.; Wang, Z. G.; Jiang, L. J.; Ruan, Y. P.; Huang, P. Q. J. Org. Chem. 2010, 75, 4619.
(320) Corey, E. J.; Kang, J. J. Am. Chem. Soc. 1981, 103, 4618.

(321) Rodriguez, A. R.; Spur, B. W. Tetrahedron Lett. 2005, 46, 3623.

(322) Becker, D.; Cyjon, R.; Cosse, A.; Moore, I.; Kimmel, T.; Wysoki, M. Tetrahedron Lett. 1990, 31, 4923.

(323) Rodriguez, A.; Nomen, M.; Spur, B. W.; Godfroid, J. J.; Lee, T. H. Tetrahedron 2001, 57, 25.

(324) Shiraishi, M.; Terao, S. J. Chem. Soc., Perkin Trans. 1 1983, 1591.

(325) Kerdesky, F. A. J.; Holms, J. H.; Schmidt, S. P.; Dyer, R. D.; Carter, G. W. Tetrahedron Lett. 1985, 26, 2143.

(326) Easton, C. J.; Ferrante, A.; Robertson, T. A.; Xia, L. Aust. J. Chem. 2002, 55, 647.

(327) Che, C.; Zhang, Z. N. Tetrahedron 2005, 61, 2187.

(328) Khrimian, A.; Oliver, J. E.; Hahn, R. C.; Dees, N. H.; White, J.; Mastro, V. C. J. Agric. Food Chem. 2004, 52, 2890.

(329) Kodama, K.; Matsui, K.; Hatanaka, A.; Ishihara, M.; Kajiwara, T. Phytochemistry 1993, 33, 1039.

(330) Mohamed, Y. M. A.; Hansen, T. V. Tetrahedron Lett. 2011, 52, 1057.

(331) Colobert, F.; Kreuzer, T.; Cossy, J.; Reymond, S.; Tsuchiya, T.; Ferrie, L.; Marko, I. E.; Jourdain, P. Synlett 2007, 2351.

(332) Yadav, J. S.; Chander, M. C.; Reddy, K. K. Tetrahedron Lett. 1992, 33, 135.

(333) Rao, A. V. R.; Reddy, E. R.; Purandare, A. V.; Varaprasad, C. V. N. S. Tetrahedron 1987, 43, 4385.

(334) Kai, K.; Takeuchi, J.; Kataoka, T.; Yokoyama, M.; Watanabe, N. Tetrahedron 2008, 64, 6760.

(335) Nakanishi, A.; Mori, K. Biosci., Biotechnol., Biochem. 2005, 69, 1007.

(336) Rowley, E. G.; Schore, N. E. J. Org. Chem. 1992, 57, 6853.

(337) Adlof, R. O.; Emken, E. A. J. Labelled Compd. Radiopharm. 1986, 23, 149.

(338) Nicolaou, K. C.; Veale, C. A.; Webber, S. E.; Katerinopoulos, H. J. Am. Chem. Soc. 1985, 107, 7515.

(339) Yao, F. M.; Li, C.; Vadivel, S. K.; Bowman, A. L.; Makriyannis, A. Bioorg. Med. Chem. Lett. 2008, 18, 5912.

(340) Gronquist, M. R.; Meinwald, J. J. Org. Chem. 2001, 66, 1075.

(341) Li, C.; Xu, W.; Vadivel, S. K.; Fan, P. S.; Makriyannis, A. J. Med. Chem. 2005, 48, 6423.

(342) Stratmann, K.; Boland, W.; Muller, D. G. Tetrahedron 1993, 49,3755 .

(343) Marron, B. E.; Spanevello, R. A.; Elisseou, M. E.; Serhan, C. N.; Nicolaou, K. C. J. Org. Chem. 1989, 54, 5522.

(344) Kerdesky, F. A. J.; Schmidt, S. P.; Brooks, D. W. J. Org. Chem. 1993, 58, 3516.

(345) Kang, B.; Britton, R. Org. Lett. 2007, 9, 5083.

(346) Suhara, Y.; Oka, S.; Kittaka, A.; Takayama, H.; Waku, K.; Sugiura, T. Bioorg. Med. Chem. 2007, 15, 854.

(347) Lapitskaya, M. A.; Vasiljeva, L. L.; Demin, P. M.; Pivnitsky, K. K. Mendeleev Commun. 2004, 287.

(348) Romanov, S. G.; Ivanov, I. V.; Shevchenko, V. P.; Nagaev, I. Y.; Pushkov, A. A.; Myasoedov, N. F.; Myagkova, G. I.; Kuhn, H. Chem. Phys. Lipids 2004, 130, 117.

(349) Vasiljeva, L. L.; Manukina, T. A.; Demin, P. M.; Lapitskaja, M. A.; Pivnitsky, K. K. Tetrahedron 1993, 49, 4099.

(350) Kigoshi, H.; Shizuri, Y.; Niwa, H.; Yamada, K. Tetrahedron 1986, 42, 3781.

(351) Groza, N. V.; Ivanov, I. V.; Romanov, S. G.; Shevchenko, V. P.; Myasoedov, N. F.; Nigam, S.; Myagkova, G. I. J. Labelled Compd. Radiopharm. 2004, 47, 11.

(352) Donaldson, W. A.; Tao, C. L. Synlett 1991, 895.

(353) Carless, H. A. J.; Batten, R. J. J. Chem. Soc., Perkin Trans. 1 1987, 1999.

(354) Ivanov, I. V.; Romanov, S. G.; Shevchenko, V. P.; Rozhkova, E. A.; Maslov, M. A.; Groza, N. V.; Myasoedov, N. F.; Kuhn, H.; Myagkova, G. I. Tetrahedron 2003, 59, 8091.

(355) Trost, B. M.; Livingston, R. C. J. Am. Chem. Soc. 2008, 130, 11970. 
(356) Furstner, A.; De Souza, D.; Parra-Rapado, L.; Jensen, J. T. Angew. Chem., Int. Ed. Engl. 2003, 42, 5358.

(357) Furstner, A.; De Souza, D.; Turet, L.; Fenster, M. D. B.; ParraRapado, L.; Wirtz, C.; Mynott, R.; Lehmann, C. W. Chem.-Eur. J. 2007, 13, 115.

(358) Corey, E. J.; Shih, C.; Shih, N. Y.; Shimoji, K. Tetrahedron Lett. 1984, 25, 5013.

(359) Haffner, T.; Nordsieck, A.; Tressl, R. Helv. Chim. Acta 1996, 79, 2088.

(360) Khanapure, S. P.; Shi, X. X.; Powell, W. S.; Rokach, J. J. Org. Chem. 1998, 63, 4098.

(361) Palazon, J. M.; Martin, V. S. Tetrahedron Lett. 1988, 29, 681.

(362) Rao, B. V.; Kumar, V. S. Tetrahedron Lett. 1995, 36, 147.

(363) Buser, H. R.; Guerin, P. M.; Toth, M.; Szocs, G.; Schmid, A.; Francke, W.; Arn, H. Tetrahedron Lett. 1985, 26, 403.

(364) Itoh, S.; Kuwahara, S.; Hasegawa, M.; Kodama, O. Biosci., Biotechnol., Biochem. 2002, 66, 1591.

(365) Romanov, S. G.; Ivanov, I. V.; Groza, N. V.; Kuhn, H.; Myagkova, G. I. Tetrahedron 2002, 58, 8483.

(366) Ivanov, I. V.; Romanov, S. G.; Groza, N. V.; Nigam, S.; Kuhn, H.; Myagkova, G. I. Bioorg. Med. Chem. 2002, 10, 2335.

(367) Brudermuller, M.; Musso, H.; Wagner, A. Chem. Ber. 1988, $121,2239$.

(368) Spinella, A.; Caruso, T.; Martino, M.; Sessa, C. Synlett 2001, 1971.

(369) Overman, L. E.; Thompson, A. S. J. Am. Chem. Soc. 1988, 110, 2248.

(370) Nigam, S. S.; Weedon, B. C. L. J. Chem. Soc. 1956, 4049.

(371) Demin, P. M.; Paceasciak, C. R. Tetrahedron Lett. 1993, 34, 4305.

(372) Rao, A. V. R.; Reddy, E. R. Tetrahedron Lett. 1986, 27, 2279.

(373) Durand, S.; Parrain, J. L.; Santelli, M. J. Chem. Soc., Perkin Trans. 1 2000, 253.

(374) Corey, E. J.; Pyne, S. G.; Su, W. Tetrahedron Lett. 1983, 24, 4883.

(375) Ghosh, A. K.; Krishnan, K. Tetrahedron Lett. 1998, 39, 947.

(376) Lellouche, J. P.; Beaucourt, J. P.; Vanhove, A. Methods Enzymol. 1990, 187, 70.

(377) Lellouche, J. P.; Aubert, F.; Beaucourt, J. P. Tetrahedron Lett. 1988, 29, 3069.

(378) Lellouche, J. P.; Aubert, F.; Noel, J. P.; Boullais, C.; Beaucourt, J. P. J. Labelled Compd. Radiopharm. 1989, 27, 473.

(379) Nicolaou, K. C.; Ladduwahetty, T.; Taffer, I. M.; Zipkin, R. E. Synthesis 1986, 344.

(380) Bindl, M.; Jean, L.; Herrmann, J.; Muller, R.; Furstner, A. Chem.-Eur. J. 2009, 15, 12310.

(381) Anorbe, B.; Martin, V. S.; Palazon, J. M.; Trujillo, J. M. Tetrahedron Lett. 1986, 27, 4991.

(382) Solladie, G.; Urbano, A.; Stone, G. B. Tetrahedron Lett. 1993, 34, 6489.

(383) Nicolaou, K. C.; Webber, S. E. Synthesis 1986, 453.

(384) Petasis, N. A.; Akritopoulou-Zanze, I.; Fokin, V. V.; Bernasconi, G.; Keledjian, R.; Yang, R.; Uddin, J.; Nagulapalli, K. C.; Serhan, C. N. Prostaglandins, Leukotrienes Essent. Fattty Acids 2005, 73, 301.

(385) Nicolaou, K. C.; Ramphal, J. Y.; Petasis, N. A.; Serhan, C. N. Angew. Chem., Int. Ed. Engl. 1991, 30, 1100.

(386) Muehldorf, A. V. Tetrahedron Lett. 1994, 35, 6851.

(387) Jahn, U.; Dinca, E. J. Org. Chem. 2010, 75, 4480.

(388) Mayer, H. Pure Appl. Chem. 1979, 51, 535.

(389) Carotenoids: Synthesis; Britton, G., Liaaen-Jensen, S., Pfander, H., Eds.; Birkhaüser Verlag: 1996; Vol. 2.

(390) Corey, E. J.; Kang, J.; Laguzza, B. C.; Jones, R. L. Tetrahedron Lett. 1983, 24, 4913.

(391) Organ, M. G.; Ghasemi, H. J. Org. Chem. 2004, 69, 695.

(392) Kobayashi, Y.; Takeuchi, A.; Hattori, H. Heterocycles 2010, 80, 811.

(393) Corey, E. J.; Ritter, K.; Yus, M.; Najera, C. Tetrahedron Lett. $1987,28,3547$.
(394) Lapitskaya, M. A.; Vasiljeva, L. L.; Kochev, D. M.; Pivnitsky, K. K. Russ. Chem. Bull., Int. Ed. 2000, 49, 549.

(395) Lapitskaya, M. A.; Vasiljeva, L. L.; Zatonsky, G. V.; Pivnitsky, K. K. Mendeleev Commun. 2000, 130.

(396) Williams, C. M.; Mander, L. N. Tetrahedron 2001, 57, 425.

(397) Adlof, R. O.; Emken, E. A. J. Labelled Compd. Radiopharm. 1987, 24, 699.

(398) Batista-Pereira, L. G.; dos Santos, M. G.; Correa, A. G.; Fernandes, J. B.; Dietrich, C. R. R. C.; Pereira, D. A.; Bueno, O. C.; Costa-Leonardo, A. M. J. Braz. Chem. Soc. 2004, 15, 372.

(399) Bayer, A.; Maier, M. E. Tetrahedron 2004, 60, 6665.

(400) Iwamoto, M.; Takagi, Y.; Kogami, K.; Hayashi, K. Agric. Biol. Chem. 1983, 47, 117.

(401) Bosshardt, H.; Schlosser, M. Helv. Chim. Acta 1980, 63, 2393.

(402) Barrot, M.; Fabrias, G.; Camps, F. Tetrahedron 1994, 50, 9789.

(403) Woollard, P. M.; Hensby, C. N.; Lascelles, P. T. J. Chromatogr. 1978, 166, 411.

(404) Leblanc, Y.; Fitzsimmons, B. J.; Zamboni, R.; Rokach, J. J. Org. Chem. 1988, 53, 265.

(405) Shevchenko, V. P.; Nagaev, I. Y.; Myasoedov, N. F. Russ. Chem. Bull., Int. Ed. 1999, 68, 859.

(406) Mazur, P.; Nakanishi, K.; Elzayat, A. A. E.; Champe, S. P. J. Chem. Soc., Chem. Commun. 1991, 1486.

(407) Braddock, D. C.; Millan, D. S.; Perez-Fuertes, Y.; Pouwer, R. H.; Sheppard, R. N.; Solanki, S.; White, A. J. P. J. Org. Chem. 2009, 74, 1835.

(408) Miyaoka, H.; Tamura, M.; Yamada, Y. Tetrahedron 2000, 56, 8083.

(409) d'Ippolito, G.; Romano, G.; Caruso, T.; Spinella, A.; Cimino, G.; Fontana, A. Org. Lett. 2003, 5, 885.

(410) Caruso, T.; Spinella, A. Tetrahedron: Asymmetry 2002, 13, 2071.

(411) Tyman, J. H. P.; Visani, N. Chem. Phys. Lipids 1997, 85, 157.

(412) Mori, K.; Ebata, T. Tetrahedron 1986, 42, 3471.

(413) Saniere, M.; Lemerrer, Y.; Barbe, B.; Koscielniak, T.; Dumas, J.; Micaslanguin, D.; Depezay, J. C. Angew. Chem., Int. Ed. Engl. 1989, 28 , 614

(414) Saniere, M.; Lemerrer, Y.; Barbe, B.; Koscielniak, T.; Depezay, J. C. Tetrahedron 1989, 45, 7317.

(415) Bell, T. W.; Claccio, J. A. Tetrahedron Lett. 1988, 29, 865.

(416) Dussault, P.; Lee, I. Q. J. Org. Chem. 1992, 57, 1952.

(417) Carvalho, J. F.; Prestwich, G. D. J. Org. Chem. 1984, 49, 1251.

(418) Spinella, A.; Caruso, T.; Coluccini, C. Tetrahedron Lett. 2002, $43,1681$.

(419) Inami, K.; Teshima, T.; Emura, J.; Shiba, T. Tetrahedron Lett. 1990, 31, 4033.

(420) Babudri, F.; Fiandanese, V.; Hassan, O.; Punzi, A.; Naso, F. Tetrahedron 1998, 54, 4327.

(421) Miyaoka, H.; Tamura, M.; Yamada, Y. Tetrahedron Lett. 1998, 39, 621 .

(422) Bulman-Page, P. C.; Ley, S. V. J. Chem. Soc., Perkin Trans. 1 1984, 1847.

(423) Ogawa, N.; Kobayashi, Y. Tetrahedron Lett. 2011, 52, 3001.

(424) Karrer, P.; Eugster, C. H. Helv. Chim. Acta 1950, 33, 1172.

(425) Karrer, P.; Eugster, C. H.; Tobler, E. Helv. Chim. Acta 1950, 33, 1349.

(426) Smith, A. B.; Lupo, A. T.; Ohba, M.; Chen, K. J. Am. Chem. Soc. $1989,111,6648$.

(427) Lin, J.; Yue, X. Y.; Huang, P.; Cui, D. X.; Qing, F. L. Synthesis 2010, 267.

(428) Ogawa, S.; Urabe, D.; Yokokura, Y.; Arai, H.; Arita, M.; Inoue, M. Org. Lett. 2009, 11, 3602.

(429) Rodriguez, J. G.; Tejedor, J. L.; Rumbero, A.; Canoira, L. Tetrahedron 2006, 62, 3075.

(430) Balas, L.; Durand, T.; Saha, S.; Johnson, I.; Mukhopadhyay, S. J. Med. Chem. 2009, 52, 1005.

(431) Oger, C.; Bultel-Ponce, V.; Guy, A.; Balas, L.; Rossi, J. C.; Durand, T.; Galano, J. M. Chem.-Eur. J. 2010, 16, 13976. 
(432) Taber, D. F.; Herr, R. J.; Gleave, D. M. J. Org. Chem. 1997, 62, 194.

(433) Papahatjis, D. P.; Nahmias, V. R.; Nikas, S. P.; Schimpgen, M.; Makriyannis, A. Chem.-Eur. J. 2010, 16, 4091.

(434) Taber, D. F.; Zhang, Z. J. Org. Chem. 2005, 70, 8093.

(435) Guiard, S.; Santelli, M.; Parrain, J. L. Synlett 2001, 553.

(436) Wei, H. X.; Truitt, C. L.; Pare, P. W. Tetrahedron Lett. 2003, 44, 831 .

(437) Millar, J. G.; Oehlschlager, A. C. J. Org. Chem. 1984, 49, 2332. (438) Heitz, M. P.; Wagner, A.; Mioskowski, C.; Noel, J. P.; Beaucourt, J. P. J. Org. Chem. 1989, 54, 500.

(439) Chemin, D.; Gueugnot, S.; Linstrumelle, G. Tetrahedron 1992, 48, 4369.

(440) Mohapatra, S.; Capdevila, J. H.; Murphy, R. C.; Hevko, J. M.; Falck, J. R. Tetrahedron Lett. 2001, 42, 4109.

(441) Taber, D. F.; Reddy, P. G.; Ameson, K. O. J. Org. Chem. 2008, 73, 3467.

(442) Schiano Moriello, A.; Balas, L.; Ligresti, A.; Cascio, M. G.; Durand, T.; Morera, E.; Ortar, G.; Di Marzo, V. J. Med. Chem. 2006, 49, 2320.

(443) Gries, R.; Khaskin, G.; Gries, G.; Bennett, R. G.; King, G. G. S.; Morewood, P.; Slessor, K. N.; Morewood, W. D. J. Chem. Ecol. 2002, 28, 2283.

(444) Hiemstra, H.; Sno, M. H. A. M.; Vijn, R. J.; Speckamp, W. N. J. Org. Chem. 1985, 50, 4014.

(445) Falck, J. R.; Reddy, L. M.; Reddy, Y. K.; Bondlela, M.; Krishna, U. M.; Ji, Y.; Sun, J.; Liao, J. K. Bioorg. Med. Chem. Lett. 2003, 13, 4011.

(446) Hickmann, V.; Alcarazo, M.; Furstner, A. J. Am. Chem. Soc. 2010, 132, 11042.

(447) Dussault, P. H.; Han, Q.; Sloss, D. G.; Symonsbergen, D. J. Tetrahedron 1999, 55, 11437.

(448) Pilard, S.; Vaultier, M. Tetrahedron Lett. 1984, 25, 1555.

(449) Jaber, J. J.; Mitsui, K.; Rychnovsky, S. D. J. Org. Chem. 2001, 66, 4679.

(450) Tietze, L. F.; Thede, K.; Schimpf, R.; Sannicolo, F. Chem. Commun. 2000, 583.

(451) Caijo, F.; Mosset, P.; Grée, R.; Audinot-Bouchez, V.; Boutin, J.; Renard, P.; Caignard, D.-H.; Dacquet, C. Bioorg. Med. Chem. Lett. 2005, 15, 4421.

(452) Chakraborty, T. K.; Tapadar, S.; Raju, T. V.; Annapurna, J.; Singh, H. Synlett 2004, 2484.

(453) Khanapure, S. P.; Wang, S. S.; Powell, W. S.; Rokach, J. J. Org. Chem. 1997, 62, 325.

(454) Raghavan, S.; Mustafa, S.; Sridhar, B. J. Org. Chem. 2009, 74, 4499.

(455) Suh, Y. G.; Min, K. H.; Lee, Y. S.; Seo, S. Y.; Kim, S. H.; Park, H. J. Tetrahedron Lett. 2002, 43, 3825.

(456) Falck, J. R.; Barma, D.; Mohapatra, S.; Bandyopadhyay, A.; Reddy, K. M.; Qi, J.; Campbell, W. Bioorg. Med. Chem. Lett. 2004, 14, 4987.

(457) Millar, J. G.; Giblin, M.; Barton, D.; Underhill, E. W. J. Chem. Ecol. 1991, 17, 911.

(458) Jacquot, C.; Wecksler, A. T.; McGinley, C. M.; Segraves, E. N.; Holman, T. R.; van der Donk, W. A. Biochemistry 2008, 47, 7295.

(459) Bestmann, H. J.; Brosche, T.; Koschatzky, K. H.; Michaelis, K.; Platz, H.; Roth, K.; Su $\beta$, J.; Vostrowsky, O.; Knauf, W. Tetrahedron Lett. 1982, 23, 4007.

(460) Taber, D. F.; You, K. J. Org. Chem. 1995, 60, 139.

(461) Hansen, T. V.; Stenstrom, Y. Synth. Commun. 2000, 30, 2549.

(462) Dasse, O.; Mahadevan, A.; Han, L.; Martin, B. R.; Marzo, V.

D.; Razdan, R. K. Tetrahedron 2000, 56, 9195.

(463) Mori, K.; Ebata, T. Tetrahedron Lett. 1981, 22, 4281.

(464) Durand, S.; Parrain, J.-L.; Santelli, M. Synthesis 1998, 1998, 1015.

(465) Jeffery, T.; Gueugnot, S.; Linstrumelle, G. Tetrahedron Lett. 1992, 33, 5757.

(466) Rezanka, T.; Nedbalova, L.; Sigler, K. Phytochemistry 2008, 69, 2391.
(467) Qi, L.; Meijler, M. M.; Lee, S.-H.; Sun, C.; Janda, K. D. Org. Lett. 2004, 6, 1673.

(468) Stoller, A.; Mioskowski, C.; Sepulchre, C.; Bellamy, F. Tetrahedron Lett. 1990, 31, 361.

(469) Jagadeesh, S. G.; Reddy, L. M.; Nasjletti, A.; Falck, J. R. Tetrahedron Lett. 2004, 45, 7111.

(470) Hulot, C.; Blond, G. 1.; Suffert, J. J. Am. Chem. Soc. 2008, 130, 5046.

(471) Hulot, C.; Amiri, S.; Blond, G. 1.; Schreiner, P. R.; Suffert, J. J. Am. Chem. Soc. 2009, 131, 13387.

(472) Gueugnot, S.; Alami, M.; Linstrumelle, G.; Mambu, L.; Petit, Y.; Larcheveque, M. Tetrahedron 1996, 52, 6635.

(473) Semeyn, C.; Blaauw, R. H.; Hiemstra, H.; Speckamp, W. N. J. Org. Chem. 1997, 62, 3426.

(474) Chakraborty, T. K.; Laxman, P. Tetrahedron Lett. 2003, 44, 4989.

(475) Kishi, N.; Maeda, T.; Mikami, K.; Nakai, T. Tetrahedron 1992, 48, 4087.

(476) Rehders, F.; Hoppe, D. Synthesis 1992, 1992, 859.

(477) Zhong, C.; Kunii, S.; Kosaka, Y.; Sawamura, M.; Ito, H. J. Am. Chem. Soc. 2010, 132, 11440.

(478) Tietze, L. F.; Bratz, M.; Pretor, M. Chem. Ber. 1989, 122, 1955.

(479) Taber, D. F.; Phillips, M. A.; Hubbard, W. C. Prostaglandins 1981, 22, 349.

(480) Meaeorg, U.; Loodman, E.; Viirlaid, S. New Activated Zn-Cucatalyst-Superior Tool for the Partial Hydrogenation of Triple Bonds; ECTOC: 1995; http://www.ch.ic.ac.uk/ectoc/ectoc_syn.html.

(481) Khrimian, A. Tetrahedron 2005, 61, 3651.

(482) Chauhan, K.; Bhatt, R. K.; Falck, J. R.; Capdevila, J. H. Tetrahedron Lett. 1994, 35, 1825.

(483) Avignon-Tropis, M.; Pougny, J. R. Tetrahedron Lett. 1989, 30, 4951.

(484) Solladie, G.; Somny, F.; Colobert, F. Tetrahedron: Asymmetry $1997,8,801$.

(485) Khrimian, A.; Klun, J. A.; Hijji, Y.; Baranchikov, Y. N.; Pet'ko, V. M.; Mastro, V. C.; Kramer, M. H. J. Agric. Food Chem. 2002, 50, 6366.

(486) Naf, F.; Decorzant, R.; Thommen, W.; Willhalm, B.; Ohloff, G. Helv. Chim. Acta 1975, 58, 1016.

(487) Navarro, I.; Mas, E.; Fabrias, G.; Camps, F. Bioorg. Med. Chem. 1997, 5, 1267.

(488) Avignontropis, M.; Treilhou, M.; Pougny, J. R.; Frechardortuno, I.; Linstrumelle, G. Tetrahedron 1991, 47, 7279.

(489) Treilhou, M.; Couderc, F. J. Labelled Compd. Radiopharm. 2001, 44, 737.

(490) Allard, M.; Barnes, K.; Chen, X. M.; Cheung, Y. Y.; Duffy, B.; Heap, C.; Inthavongsay, J.; Johnson, M.; Krishnamoorthy, R.; Manley, C.; Steffke, S.; Varughese, D.; Wang, R. F.; Wang, Y.; Schwartz, C. E. Tetrahedron Lett. 2011, 52, 2623.

(491) Sondengam, B. L.; Fomum, Z. T.; Charles, G.; Macakam, T. J. Chem. Soc., Perkin Trans. 1 1983, 1219.

(492) Stephenson, L. M.; Gemmer, R. V.; Current, S. P. J. Org. Chem. 1977, 42, 212.

(493) Aerssens, M. H. P. J.; Vanderheiden, R.; Heus, M.; Brandsma, L. Synth. Commun. 1990, 20, 3421.

(494) Solladie, G.; Stone, G. B.; Andres, J. M.; Urbano, A. Tetrahedron Lett. 1993, 34, 2835.

(495) Solladie, G.; Kalai, C.; Adamy, M.; Colobert, F. Tetrahedron Lett. 1997, 38, 6917.

(496) Chemin, D.; Linstrumelle, G. Tetrahedron 1992, 48, 1943.

(497) Urabe, D.; Todoroki, H.; Masuda, K.; Inoue, M. Tetrahedron 2012, 68, 3210.

(498) Rodriguez, A. R.; Spur, B. W. Tetrahedron Lett. 2012, 53, 1912.

(499) Rodriguez, A. R.; Spur, B. W. Tetrahedron Lett. 2012, 53, 86.

(500) Petasis, N. A.; Yang, R.; Winkler, J. W.; Zhu, M.; Uddin, J.; Bazan, N. G.; Serhan, C. N. Tetrahedron Lett. 2012, 53, 1695.

(501) Yang, R. Ph.D. Dissertation, University of Southern California, 2005.

(502) Petasis, N. A.; Heights, H. U.S. Patent US0228047 A1, 2005. 
(503) Millar, J. G.; Underhill, E. W. Can. J. Chem. 1986, 64, 2427. (504) Brudermüller, M.; Musso, H. Angew. Chem., Int. Ed. Engl. 1988, 27, 298.

(505) Svatos, A.; Attygalle, A. B.; Meinwald, J. Tetrahedron Lett. 1994, 35, 9497.

(506) Unelius, C. R.; Liblikas, I.; Mozuraitis, R. Acta Chem. Scand. 1998, 52, 930.

(507) Crombie, L.; Holloway, S. J. J. Chem. Soc., Perkin Trans. 1 1985, 2425.

(508) Crombie, L.; Morgan, D. O. J. Chem. Soc., Chem. Commun. 1988, 558 .

(509) Crombie, L.; Morgan, D. O. J. Chem. Soc., Perkin Trans. 1 1991, 581.

(510) Crombie, L.; Morgan, D. O.; Smith, E. H. J. Chem. Soc., Perkin Trans. 1 1991, 567.

(511) Meyer, M. P.; Klinman, J. P. Tetrahedron Lett. 2008, 49, 3600.

(512) Shimazaki, T.; Kobayashi, Y.; Sato, F. Chem. Lett. 1988, 1785.

(513) Attygalle, A. B.; Jham, G. N.; Svatos, A.; Frighetto, R. T. S.; Meinwald, J.; Vilela, E. F.; Ferrara, F. A.; Uchoafernandes, M. A. Tetrahedron Lett. 1995, 36, 5471.

(514) Mori, K. Biosci., Biotechnol., Biochem. 2009, 73, 2727.

(515) Blackburn, L.; Kanno, H.; Taylor, R. J. K. Tetrahedron Lett. 2003, 44, 115

(516) Svirskaya, P. I.; Leznoff, C. C.; Roelofs, W. L. Synth. Commun. 1980, 10, 391.

(517) Brown, H. C.; Bigley, D. B.; Arora, S. K.; Yoon, N. M. J. Am. Chem. Soc. 1970, 92, 7161.

(518) Godjoian, G.; Singaram, B. Tetrahedron Lett. 1997, 38, 1717.

(519) Zweifel, G.; Polston, N. L. J. Am. Chem. Soc. 1970, 92, 4068.

(520) Brown, H. C.; Liotta, R.; Scouten, C. G. J. Am. Chem. Soc. 1976, 98, 5297.

(521) Sato, F.; Kobayashi, Y. Synlett 1992, 849.

(522) Pelter, A.; Singaram, S.; Brown, H. Tetrahedron Lett. 1983, 24 1433.

(523) Kulinkovich, O. G.; Sviridov, S. V.; Vasilevski, D. A. Synthesis 1991, 234.

(524) Lara-Ochoa, F.; Espinosa-Perez, G. Tetrahedron Lett. 2007, 48, 7007.

(525) Obora, Y.; Moriya, H.; Tokunaga, M.; Tsuji, Y. Chem. Commun. 2003, 2820.

(526) Uhl, W. Coord. Chem. Rev. 2008, 252, 1540.

(527) Alexakis, A.; Duffault, J. M. Tetrahedron Lett. 1988, 29, 6243.

(528) Gensler, W. J.; Bruno, J. J. J. Org. Chem. 1963, 28, 1254.

(529) Utimoto, K.; Uchida, K.; Yamaya, M.; Nozaki, H. Tetrahedron Lett. 1977, 3641.

(530) Meilert, K.; Brimble, M. A. Org. Biomol. Chem. 2006, 4, 2184.

(531) Marko, I. E.; Bayston, D. J. Tetrahedron 1994, 50, 7141.

(532) Zweifel, G.; Clark, G. M.; Whitney, C. C. J. Am. Chem. Soc. 1971, 93, 1305.

(533) Clements, A. H.; Vandenen, R.; Frost, D. J.; Hoogenho., K; Nooi, J. R. J. Am. Oil Chem. Soc. 1973, 50, 325.

(534) Rink, C.; Navickas, V.; Maier, M. E. Org. Lett. 2011, 13, 2334. (535) Luthy, C.; Konstantin, P.; Untch, K. G. J. Am. Chem. Soc. 1978, 100,6211 .

(536) Nicolaou, K. C.; Marron, B. E.; Veale, C. A.; Webber, S. E.; Serhan, C. N. J. Org. Chem. 1989, 54, 5527.

(537) Denmark, S. E.; Yang, S. M. J. Am. Chem. Soc. 2002, 124, 15196.

(538) Trost, B. M.; Frederiksen, M. U.; Papillon, J. P. N.; Harrington, P. E.; Shin, S.; Shireman, B. T. J. Am. Chem. Soc. 2005, 127, 3666.

(539) Huang, W.; Pulaski, S. P.; Meinwald, J. J. Org. Chem. 1983, 48, 2270 .

(540) Tallman, K. A.; Roschek, B.; Porter, N. A. J. Am. Chem. Soc. 2004, 126, 9240.

(541) Hnarnachari, V. K.; Xie, X.; Zhu, S.; Wei, H. X.; Pare, P. W. Nat. Prod. Commun. 2007, 2, 1019.

(542) Tallman, K. A.; Rector, C. L.; Porter, N. A. J. Am. Chem. Soc. 2009, 131, 5635 .
(543) Bhatt, R. K.; Chauhan, K.; Wheelan, P.; Murphy, R. C.; Falck, J. R. J. Am. Chem. Soc. 1994, 116, 5050.

(544) De Sousa, P. T.; Taylor, R. J. K. J. Braz. Chem. Soc. 1993, 4, 64.

(545) Thompson, A. F. In Deuterium Labeling in Organic Chemistry; Appleton-Century-Crofts Educational Division/Meredith Corporation: New York, 1971; pp 308-319.

(546) Meilert, K.; Brimble, M. A. Org. Lett. 2005, 7, 3497.

(547) van Summeren, R. P.; Feringa, B. L.; Minnaard, A. J. Org. Biomol. Chem. 2005, 3, 2524. 